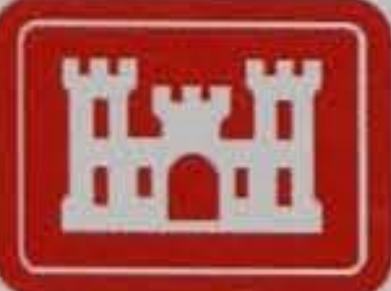

US Army Corps of Engineers Engineer Research and Development Center

\title{
Application of DEM to Micro-Mechanical Theory for Large Deformations of Granular Media
}

David A. Horner and John F. Peters

August 2000 


\section{Application of DEM to Micro-Mechanical Theory for Large Deformations of Granular Media}

by David A. Horner, John F. Peters

Geotechnical Laboratory

U.S. Army Engineer Research and Development Center

3909 Halls Ferry Road

Vicksburg, MS 39180-6199

Final report

Approved for public release; distribution is limited

Prepared for U.S. Army Engineer Research and Development Center 3909 Halls Ferry Road

Vicksburg, MS 39180-6199 


\section{Engineer Research and Development Center Cataloging-in-Publication Data}

Horner, David A.

Application of DEM to micro-mechanical theory for large deformations of granular media / by David A. Horner, John F. Peters ; prepared for U.S. Army Engineer Research and Development Center.

133 p. : ill. ; $28 \mathrm{~cm}$. -- (ERDC/GL ; TR-00-7)

Includes bibliographic references.

1. Particle size determination -- Mathematical models. 2. Soils --Testing -- Mathematical models. 3. Granular materials -- Mathematical models. I. Peters, John F. II. United States. Army. Corps of Engineers. III. U.S. Army Engineer Research and Development Center. IV. Geotechnical Laboratory (U.S.) V. Title. VI. Series: ERDC/GL TR ; 00-7.

TA7 E8 no.ERDC/GL TR-00-7 


\section{Preface}

The study reported herein was conducted at the U.S. Army Engineer Research and Development Center (ERDC), Geotechnical Laboratory (GL), Mobility Systems Division (MSD), Vicksburg, MS, under the USAE RDT\&E 6.1 Research Work Item Code 0060MP, "Vehicle Terrain Interaction." The work was conducted between January 1996 and December 1998.

This report, was a dissertation in partial fulfillment of the requirements for the degree of Doctor of Philosophy from the University of Michigan at Ann Arbor, was written by Dr. David A. Horner. Dr. John F. Peters served as technical advisor and thesis reader. MSD personnel supporting this study were Mses. Glenda Brandon and Mary A. Dungan.

The study was conducted under general supervision of Dr. William F. Marcuson III, GL, Dr. William E. Willoughby, Acting Chief, MSD, and Dr. Michael J. O'Connor, Director, GL.

At the time of publication of this report, Dr. James R. Houston was Director, ERDC, and COL James S. Weller, EN, was Commander. 


\section{ACKNOWLEDGEMENTS}

I would like to express my sincere gratitude to Professor Roman D. Hryciw for serving as Chairman, the Dissertation Committee, and providing guidance and warm hospitality. Thanks, also to Dr. John F. Peters for immeasurable assistance as mentor, for friendship, guidance and for graciously answering all my questions, no matter how many times I asked. I would also like to thank Professor Richard D. Woods, Professor E. Benjamin Wylie, and Professor Richard A. Scott for their assistance and guidance in producing this dissertation. I especially want to thank Scott Raschke, Ali Ghalib, and Bob Fischer who designed and built the equipment used in Chapter 4, and provided special assistance in performing many of the tests described throughout this dissertation. The author gratefully acknowledges the support of the United States Army Engineer Research and Development Center (ERDC), Waterways Experiment Station. I would like to thank all my friends who supported me. In particular, I'd like to thank Dr. Mike Stevens for giving me the capability to visualize my data, Ms. Teresa Lee for ensuring that the computer simulations ran in a timely manner, Mr. Alex Carrillo for his assistance in ensuring effective use of the computer resources, Mr. George McKinley for sharing his knowledge on computer programming and code debugging, and Mrs. Susan Sippel for assisting in the preparation of this report. Thanks to my wife for all the proofreading, typing, waiting up endless nights, taking care of the kids like a single parent, etc. 
PREFACE

ACKNOWLEDGMENTS ............................................................. ii

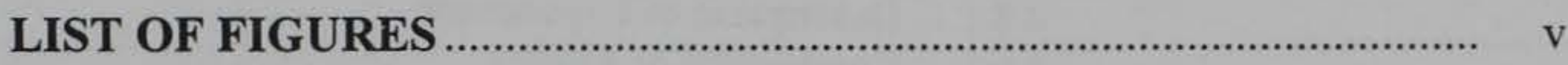

LIST OF TABLES ................................................................................ viii

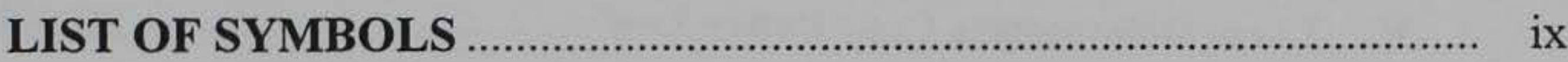

\section{CHAPTER}

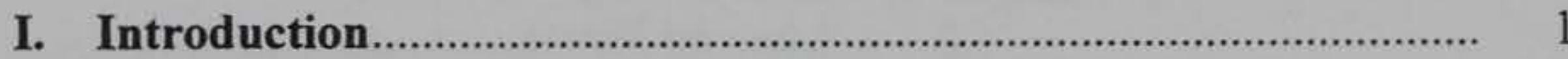

1.1 Background ............................................. 1

1.2 Towards a Continuum Mechanics Model for

Granular Media ................................................................. 1

1.3 Discrete Element Method .................................. 7

1.4 Smoothed Particle (Hydrodynamic) Codes................... 9

1.5 Objective of Research .......................................................... 9

1.6 Dissertation Organization ................................. 10

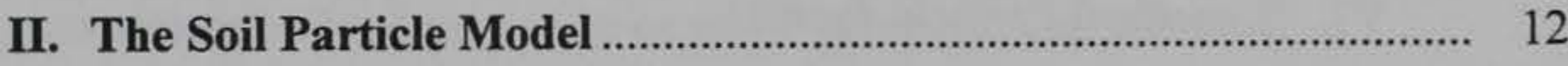

2.1 Stability of DEM......................................................... 14

2.2 Particle Force Calculations................................. 15

2.2.1 Normal Force.............................. 17

2.2.2 Shear Forces.................................. 18

2.3 Energy Damping ....................................................... 19

2.3.1 Hysteretic Contact Law ............................... 20

2.3.2 Variable Viscous Local Damper .............. 23

2.4 Particle Motion ............................................ 23

2.5 Optimization of Particle Code........................................ 24

III. Continuum Representation of Discrete Particle Systems .............. 26

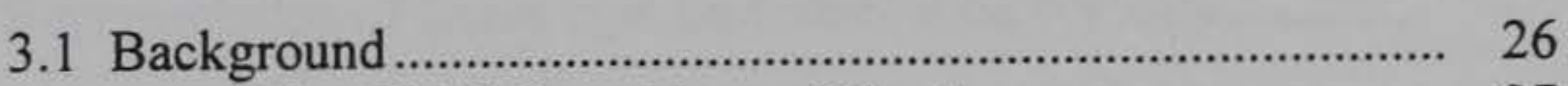

3.2 Smoothing Field Quantities and Strain ................... 27

3.2.1 Implied Average Volume..................... 28

3.2.2 Computation of Gradients........................... 30

3.2.3 Momentum, Velocity, and $\quad$ Velocity Gradient.......................................... 31

3.2.4 Sampling Size Effects....................... 31

3.2.5 Strain ........................................ 33

3.3 Kinematics of Averaged Quantities ....................... 35

IV. Large Deformation Analyses using DEM ………...................... 41

4.1 Introduction.................................................................... 41

4.2 Previous work on DEM Validation..................................... 41 
4.3 Effects of Representation Grain Size Distribution.......... 42

4.4 Soil Plowing Simulation............................... 43

4.5 Plowing Experiment.......................................................... 44

4.6 Stimulation Description.............................. 48

4.7 Results and Comparisons between Experiment and

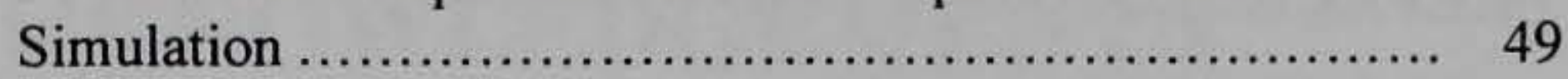

4.8 Trap Door Simulations................................................. 52

4.8.1 Description of Experiment.......................... 52

4.8.2 Comparison of Results.................... 52

4.8.3 Conclusions.............................. 53

v. Large Deformation Constitutive Law ……………..................... 65

5.1 Governing Equations for Smoothed Particle System ........ 65

5.2 Analysis of DEM Plowing Simulation............................. 67

5.2.1 Material Points........................... 67

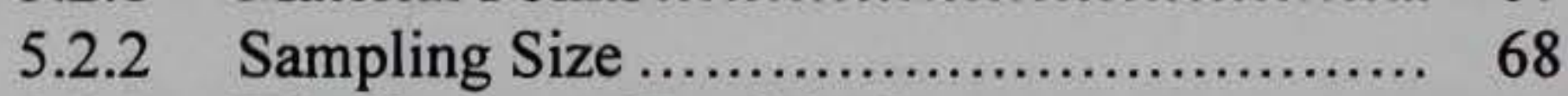

5.2.3 Distribution of Particle Contacts and Forces .............................. 68

5.2.4 Estimate of Coordination Number .............. 69

5.2.5 Average Normal Vector.............................. 73

5.3 Development of the Average Particle ................................. 76

5.4 Evaluation of the Smoothed Model ..................................... 78

VI. Smoothed Particle System …..................................................... 98

6.1 Development of the Smoothed Particle System .................. 98

6.2 Plow Simulation.............................................................. 102

VII. Summary and Conclusions......................................................... 107

7.1 Significant Finding ............................................................ 107

7.2 Need for Future Research .............................................. 108

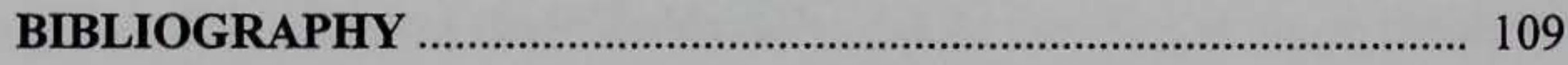

APPENDIX 


\section{LIST OF FIGURES}

\section{Figure}

1.1 Soil Plowing (a) Finite Element Analysis (b) Laboratory

Experiment

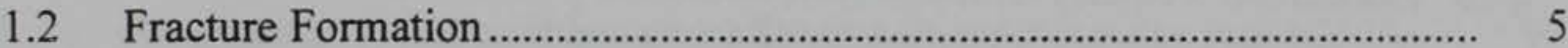

1.3 Sand Flow through and Hour Glass ....................................................... 6

2.1 Examples of DEM (a) Soil Plowing and (b) Trap Door Simulations.......... 13

$2.2 \quad$ Particle Contact Interaction Model......................................................... 16

2.3 Hysteretic Contact Law .................................................................... 21

2.4 Single processor CRAY Y-MP CPU time-per-time step vs. number of particles for the three phases of single processor optimization............... 25

3.1 Characteristic Length of the Kernel Function ........................................ 30

3.2 Effects of Sampling Size on Gradient Estimate ....................................... 32

3.3 Deformed Material and Spatial Grid ......................................................... 33

3.4 Labeling of Particles and their Contacts................................................. 37

4.1 Treasure Island Grain Size Distribution Curve ........................................ 44

4.2 Probability of Particle Size Occurrence ..................................................... 45

4.3 Particle Packing using (a) One Grain Size (b) Five Grain Sizes.................. 46

4.4 Effects of Representation of Grain Size Distribution on Porosity .............. 47

4.5 Effects of Representation of Grain Size Distribution on Coordination Number........................................................................................... 47

4.6 The Plowing Problem .............................................................................. 50

4.7 Plow Experiment at (a) $0 \mathrm{~cm}$ Displacement, (b) $1 \mathrm{~cm}$ Displacement, (c) $2 \mathrm{~cm}$ Displacement...................................................................... 54

4.8 Comparison of Particle Traces over $2 \mathrm{~cm}$ of Plowing............................... 55

4.9 Experimental and Simulation Particle Velocities................................... 55

4.10 Comparison of Velocity Magnitudes Particle during $1 \mathrm{~cm}$ of Plowing (a) Simulation (b) Experiment. 
4.11 Comparison of Velocity Magnitudes Particle during $2 \mathrm{~cm}$ of Plowing

(a) Simulation (b) Experiment

4.12 Plow Forces 58

4.13 Shear Deformation Rate at (a) $1 \mathrm{~cm}$ of Plowing (b) $2 \mathrm{~cm}$ of Plowing. 59

4.14 Rotation Rate at (a) $1 \mathrm{~cm}$ of Plowing (b) $2 \mathrm{~cm}$ of Plowing 60

4.15 Trap Door Problem 61

4.16 Surface Profile at 2 Seconds for (a) Ottawa 20-30 (b) Glass Beads (c) Simulation

4.17 Horizontal Velocity Gradient with respect to y at (a) 0.5 Seconds (b) 1.0 Seconds (c) 1.5 Seconds (d) 2.0 Seconds

4.18 Horizontal Velocity Gradient with respect to y at (a) 2.5 Seconds (c) 3.0 Seconds (c) 3.5 Seconds (d) 4.0 Seconds 64

5.1 Initial Location of Material Points 70

5.2 Effects of Sample on Jacobian Determinant ......................................... 71

5.3 Effects of Sampling Size on Shear Strain............................................... 71

5.4 Effects of Sampling Size on Shear Stress............................................... 72

5.5 Spatial and Material Coordinate Systems............................................... 72

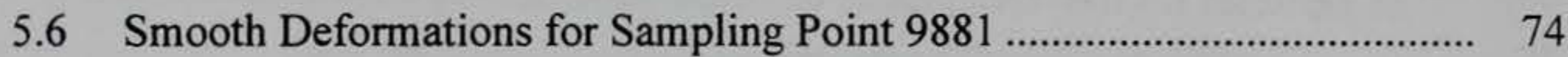

5.7 Distribution of Normal Forces by Contact Angle at $5 \mathrm{~mm}$ Plowing ........... 74

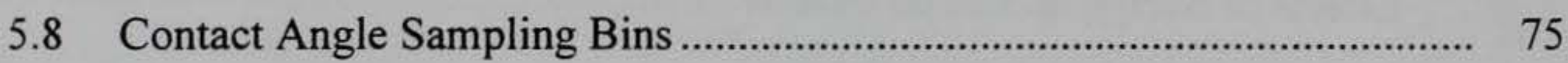

5.9 Distribution of Normal Force by Sampling Bins at $5 \mathrm{~mm}$ Plowing ............ 76

5.10 Distribution of Average Normal Force by Percentile.............................. 77

5.11 Average Normal Force by Contact Angle Bins During Plowing .............. 77

5.12 Coordination Number Vs. Void Ratio from Chang, 1989......................... 79

5.13 Relationship of Coordination Number and Mean Stress ........................... 79

5.14 Distribution of Contact Angles During Plowing …................................. 81

5.15 Conceptual Design of Spoke Model....................................................... 82

5.16 Distribution of Contact Angles from Smoothed Spoke System after $5 \mathrm{~mm}$ of Plowing ............................................................................ 84

5.17 Average Normal Force for Material Point 9310 ....................................... 86

5.18 Average Normal Force for Material Point 9811 ..................................... 86

5.19 Limits on Average Normal Force for Material Point 9515 _..................... 87

5.20 Average Normal Force for Material Point 9505 .................................... 87

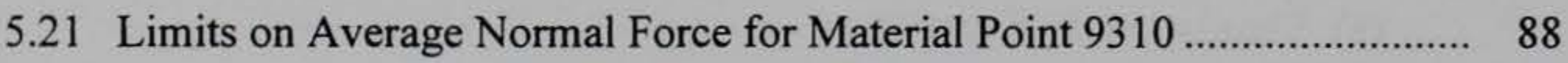


5.22 Limits on Average Normal Force for Material Point 9811 ...................... 88

5.23 Limits on Average Normal Force for Material Point 9515 ...................... 89

5.24 Limits on Average Normal Force for Material Point 9505 ...................... 89

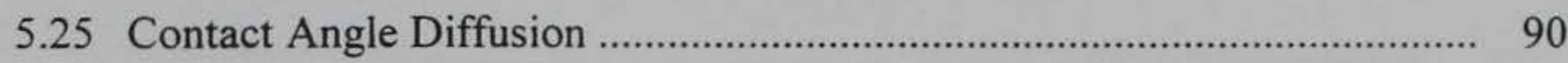

5.26 Comparison of Force Limits for DEM and Smoothed System, Point 9811

5.27 Distribution of Contact Angles from Smooth System with Diffusion ........ 91

5.28 Comparison DEM and Smoothed System Shear Stress for Point $9310 \ldots \ldots .91$

5.29 Comparison DEM and Smoothed System Shear Stress for Point 9811 ..... 92

5.30 Comparison DEM and Smoothed System Shear Stress for Point 9515 ..... 92

5.31 Comparison DEM and Smoothed System Shear Stress for Point 9505 ..... 93

5.32 Comparison DEM and Smoothed System Stress Path for Point 9310 ....... 93

5.33 Comparison DEM and Smoothed System Stress Path for Point 9811 ....... 94

5.34 Comparison DEM and Smoothed System Stress Path for Point 9515 ....... 94

5.35 Comparison DEM and Smoothed System Stress Path for Point 9505 ....... 95

5.36 Comparison DEM and Smoothed System Stress Path for Point 9310 ....... 95

5.37 Comparison DEM and Smoothed System Stress Path for Point 9811 ....... 96

5.38 Comparison DEM and Smoothed System Stress Path for Point 9515 ....... 96

5.39 Comparison DEM and Smoothed System Stress Path for Point 9505 ....... 97

6.1 Smoothed Particle System ...................................................................... 99

6.2 One Particle Example of a Smoothed Particle System ............................. 103

6.3 Smooth Particle System Plowing Simulation, Grid Size $=5 \mathrm{~mm}$............ 104

6.4 Smooth Particle System Plowing Simulation, Grid Size $=2.5 \mathrm{~mm}$........... 105

6.5 Effects of Grid Size on Particle Displacement ........................................ 106 


\section{TABLES}

4.1 Number of Particles .............................................................................. 43

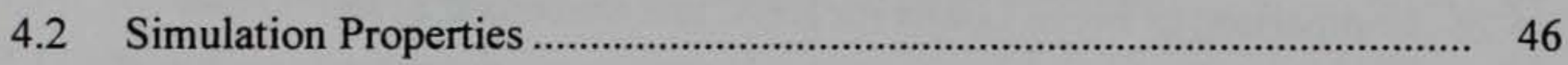

4.3 Discrete Ottawa 20-30 Grain Size Distribution....................................... 47

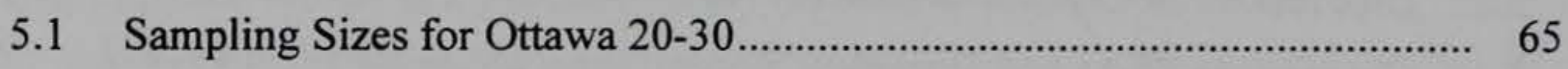

A.1 Scaling Relationships for Centrifuge Test............................................. A6 


\section{LIST OF SYMBOLS}

$b_{i}$

$c$

C

$C_{H}$

$\Delta C$

$c^{0}$

$c^{-}, c^{+}$

D

$D_{x}$

$D_{i j}$

$\delta$

$\delta_{m}$

$\delta_{L}$

$e_{i}$

EV

$E_{I J}$

$\dot{E}_{I J}$

$f_{i}$

$F_{i J}$

$\dot{F}_{i J}$

$k, K$

$K_{s}$

$L_{i j}$

$L_{s}$

$l$

$M$

$M\left(x-x^{\prime}\right)$

$m$

$\bar{m}(x)$

$N$

$n$

$n_{i} j$
$=$ Body Acceleration

$=\mathrm{Viscous}$ Damping Coefficient

$=$ Coordination Number

$=$ Green's Deformation Tensor

$=$ Change in Property as a Result of Diffusion

$=$ Smooth Value of the Property at the Point of Interest

$=$ Smooth Value Property

$=$ Diffusion Coefficient

$=$ Particle Diameter

$=$ Rate of Deformation Tensor

$=$ Contact Displacement

=Maximum Contact Displacement

$=$ Kronker Delta Function

$=$ Normal Contact Unit Vector

$=$ Expected Value

$=$ Lagrangian Strain Tensor

$=$ Lagrangian Strain Rate Tensor

$=$ Contact Force

$=$ Deformation Gradient Tensor

$=$ Deformation Gradient Rate Tensor

$=$ Contact Spring Stiffness

$=$ Number of Contacts

$=$ Spatial Velocity Gradient

$=$ Number of Contacts

$=$ Contact Length

$=$ Number of Particle Sizes

$=$ Positive Weighting Function

$=$ Particle Mass

$=$ Average Mass

$=$ Number of Particles

$=$ Hysteretic Conyct Law Power

$=$ Fabric Tensor 


$\begin{array}{ll}\pi_{c}, \pi_{k} & =\text { Dimensionless Pi-terms } \\ \bar{p}_{i}(x) & =\text { Average Momentum } \\ \phi & =\text { Kernel Weighting Function } \\ R & =\text { Particle Radius } \\ \rho_{s} & =\text { Weighting Density } \\ \bar{\sigma}_{i j} & =\text { Average Cauchy Stress } \\ \sigma^{2} & =\text { Standard Deviation } \\ t_{i} & =\text { Shear Contact Unit Vector } \\ t_{c r} & =\text { Critical Time Step } \\ \Delta t & =\text { Time Step } \\ V & =\text { Particle Volume } \\ v_{i}^{n} & =\text { Normal Contact Velocity } \\ v_{i}^{s} & =\text { Shear Contact Velocity } \\ v_{i}^{r} & =\text { Relative Contact Velocity } \\ w_{i j} & =\text { Spin Tensor } \\ \ddot{X}_{i}^{A} & =\text { Particle Acceleration } \\ \dot{X}_{i}^{A} & =\text { Particle Velocity } \\ X_{i}^{A} & =\text { Particle Location }\end{array}$




\title{
CHAPTER I
}

\author{
Introduction
}

\subsection{Background}

Numerical models are critical to effective design and planning of engineered systems. Computers allow scientists and engineers to simulate the performance of engineered systems with far greater flexibility and less cost than can be achieved through physical experiments. Quantities that are difficult or impossible to measure experimentally can be accurately estimated in simulations. Simulated experiments can be repeated with variation of parameters to isolate cause-and-effect relationships that are important to improving designs. With accurate mathematical models, resources for physical models can be concentrated on prototypes that are thought to be particularly promising or used to verify certain critical modeling assumptions.

The effectiveness of models depends on how well relevant physical phenomenon is represented mathematically. Many important problems in soil mechanics and particulate physics involve large discontinuous deformations, which are beyond the capabilities of numerical simulations based on continuum mechanics. Examples are soil plowing, pentrometers, pile driving, soil-tire interactions, hopper flows, mixing of powders, and mass movements by avalanche. Continuum formulations do not exist for such a wide range of behavior, particularly in the case of frictional materials such as sand [52]. In a particular situation, soil may deform as a solid, flow as a fluid, or behave as individual particles. All of these "phases" play important roles in the mechanical behavior, yet at present no single model exists that can account for these different modes of soil behavior. In the absence of such a model, a large and important class of soil mechanics problems lies beyond the reach of mathematical modeling.

\subsection{Towards a Continuum Mechanics Model for Granular Media}

Well-known methods of numerical approximation such as finite differences, finite elements or boundary elements have their origins in continuum descriptions of the media. Therefore, these methods are limited in principle by the assumptions of continuum mechanics. Historically, granular media have been treated, by Civil Engineers, as elastic or plastic solids because such descriptions fit expected design behavior. In fact, in many situations involving excavation, vehicle mobility, and material handling a fluid mechanics description for the soil may be more appropriate. 
The principal difficulty in applying solid mechanics continuum theory to particulate media comes from the mathematical description of the kinematics that defines the movement of material "particles" within the continuum. The traditional use of continuum mechanics is limited to strains with continuous deformation. Figure 1.1 (a) shows the results from a finite element model of a soil plowing experiment. The continuous deformations required by the finite element formulation do not capture the kinematics of motion of the real soil system, which are illustrated in Figure 1.1 (b). Movement must obey compatibility relationships that preclude formation of slip planes and motions are restricted to an affine mapping in which each point in the initial configuration can be mapped into the deformed configuration as shown in Figure 1.2. The deformations thus defined represent topologically equivalent configurations because deformation from one configuration to another is continuous and a one-to-one mapping exists. A continuum can deform such that in the vicinity of any arbitrary point a second point can be found at a sufficiently small distance, $\varepsilon$, in the initial configuration so that the points are separated by less $\delta$ than in the deformed configuration. By contrast, when a discontinuity exists or a crack forms, two points can exist at the same initial location $(\varepsilon=0) \delta$ yet can be non-zero.

In fact, the preceding description of a continuum is somewhat restrictive because finite discontinuities can exist provided relevant interface conditions are prescribed. For example, the Goodman joint element [21] used in finite element analyses is intended for such interface conditions. In fracture mechanics, discontinuities arise based on predefined fracture criteria that can be modeled as an evolving material topology. In these cases where compatibility breaks down, a mapping can exist between initial and deformed configuration by introducing conditions for the discontinuity. For example, while two points may initially lie at virtually the same location they may lie on different sides of some defined line; and, hence can be mapped to the new configuration uniquely.

While the mapping between points on a discontinuity presents no special difficulties in principle, analysis of such points is complicated because under large deformations, points along the discontinuity that are initially in "contact" no longer interact mechanically after relative movements exceeding a grain size in magnitude. Thus normal and shear tractions carried by pairs of points initially, must be carried by different pairs after deformation. As a result, the constitutive equation that relates traction at the interface to displacement along the interface is more difficult to define because the traction no longer depends on the distance between the fixed material points A and B and the stress state is not a function of the deformation alone.

Finally, there is the question of defining the discontinuity. In fracture mechanics, the formation and propagation of a fracture is controlled by a fracture criteria. No such criteria yet exist for shear bonding in soils. The continuum description breaks down at the point of shear bonding (e.g. Valanis and Peters) [52] leaving the problem ill-posed. To construct a suitable theory is needed. To create such a theory, a better view of the micro mechanics is needed.

From the preceding discussion it may be concluded that the difficulties with describing soil, as a continuum are significant. The continuum description at best applies to the particles themselves, whereas in a particulate media discontinuities are the predominant feature. The behavior of the mass is controlled by the interactions between particles or groups of particles and not the character of the particles themselves. Thus, while the finite number of discontinuities in 
a continuum can be dealt with through application of special interface relationships, the behavior of a particulate mass is completely dominated by interactions at interfaces. 

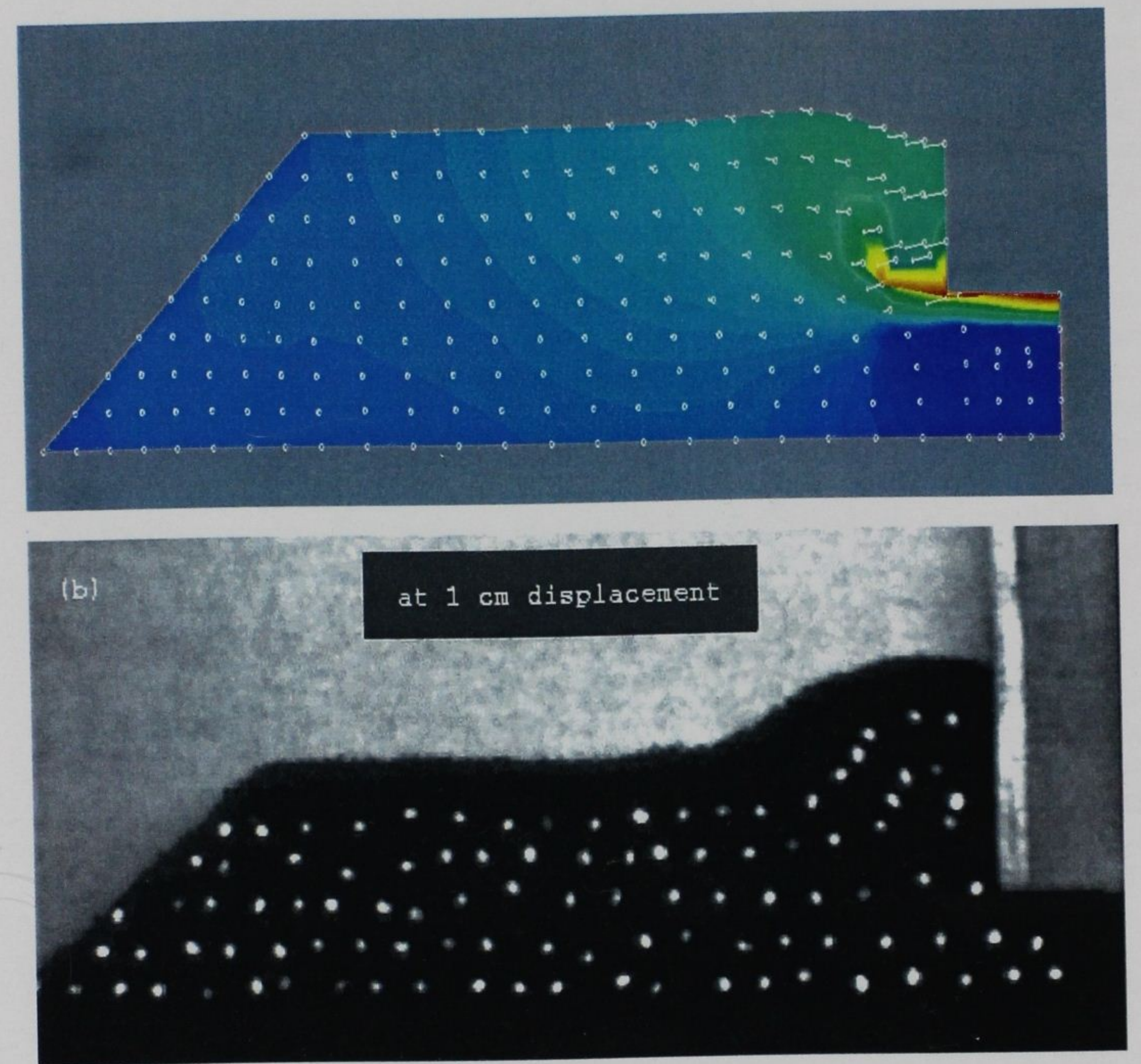

Figure 1.1: Soil Plowing (a) Finite Element Analysis without provision for discontinuity in deformation (b) Laboratory Experiment 

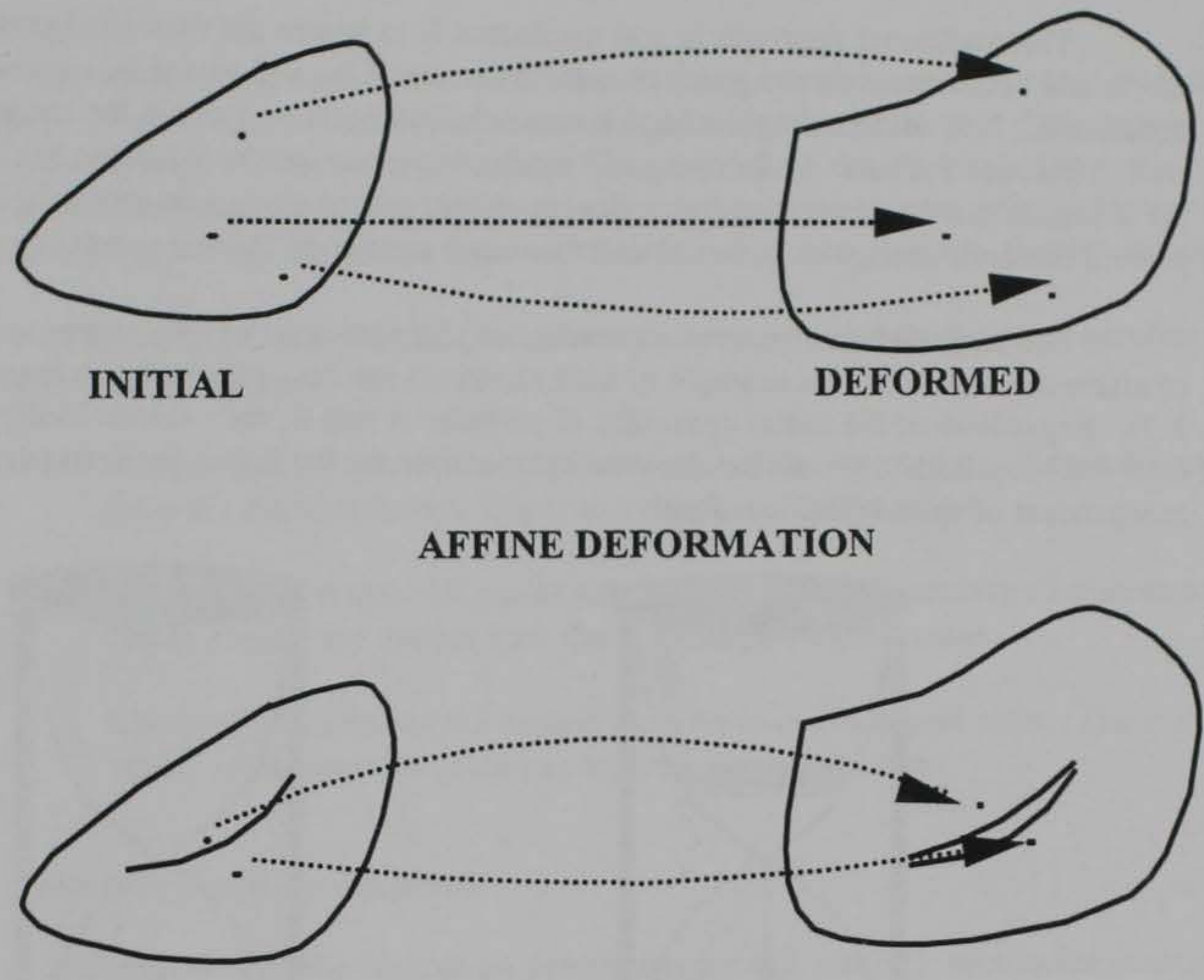

WITH DISCONTINUITY

Figure 1.2: Fracture Formation 
The traditional approach in soil mechanics is to ignore the individual particles in the media and concentrate on the group or mass behavior of the soil grains as a homogenized continuum. Not withstanding the logical contradiction contained within the model, it has served well in practice because: 1) for many soil mechanics problems deformations are small, and 2) the deformations occur over spatial scales much larger than the largest individual particle. The present research deals with deformations for which neither of the two conditions is met.

For large deformations the movement of soil particles can defy mapping by any notion of affine deformation. An example of such motion is the flow of sand in an hourglass (Figure 1.3). Regardless of the initial proximity of particles A and B, they are decidedly disconnected in their final locations. For all but the smallest movements, the forces between particle pairs are independent of their initial locations.
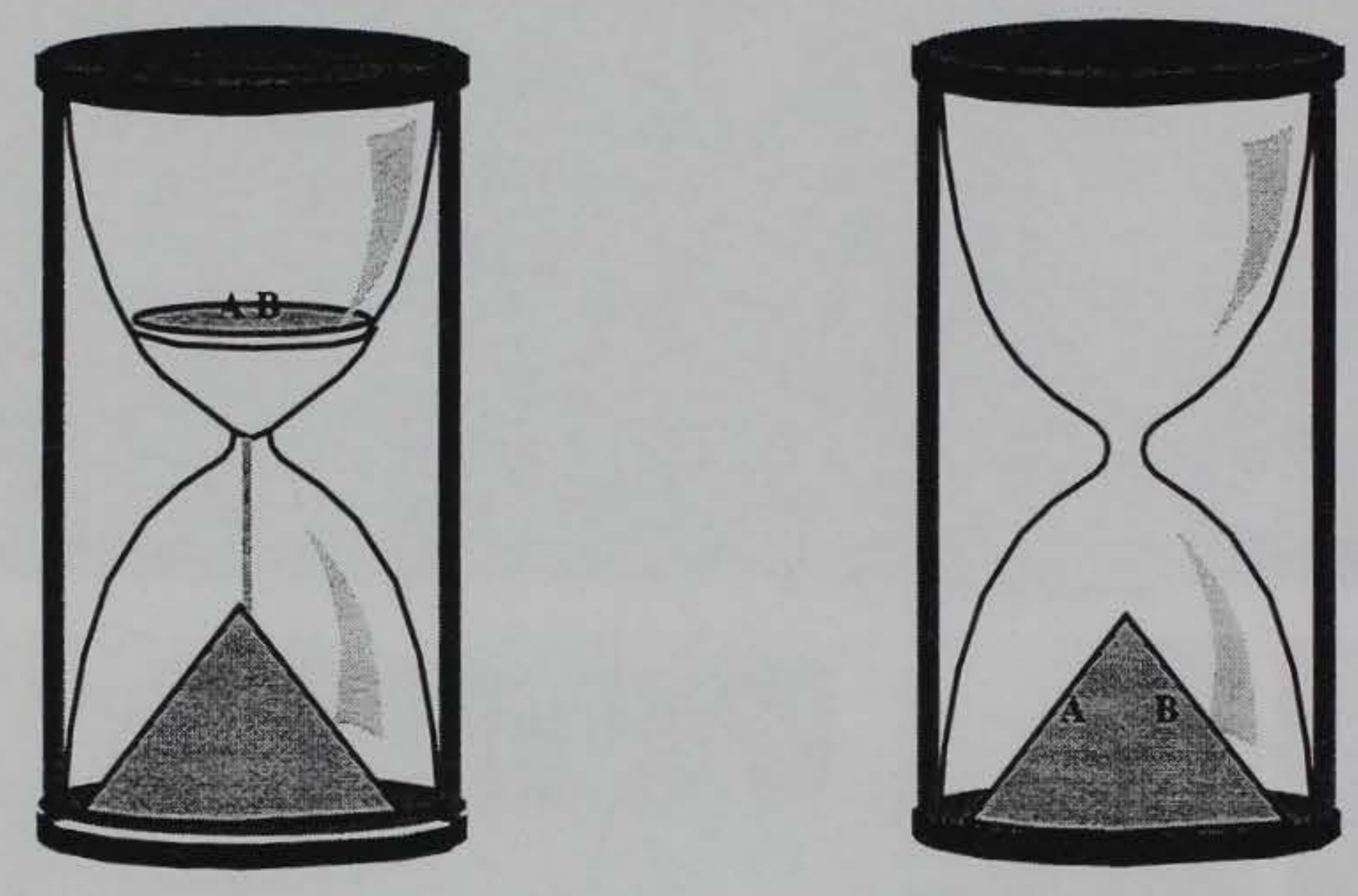

Figure 1.3: Sand Flow through an Hour Glass

Further, the multiphase aspect of sand behavior is evident. In either the top or bottom chambers of the hourglass the sand exists essentially as a solid. The sand flowing from the top chamber behaves as a fluid. In the free-fall portion of the hourglass, the discrete nature of the particles becomes evident. Upon striking the pile in the bottom chamber the sand reverts to a fluid as it flows down the slope of the sand pile. Finally, the sand returns to a solid state as it comes to rest.

In a fluid state, individual particle movements defy meaningful description. For fluids in general, traction do not depend on the details of individual particle motions but rather on timeaveraged deformation rates. Accordingly, the traction represents a time-average of individual particle forces. 
However, the fluid model is unsatisfactory for particulate media for two reasons. First, for small deformations, particles behave as solids whereby the stress state does depend on relative movements between adjacent grains. Thus, the fluid description is appropriate only when deformations are large. Secondly, the relationship between inter-particle force and movement is generally rate independent and therefore does not possess a simple viscosity law.

In summary, it appears that particulate media poses three fundamental problem when viewed from the standpoint of a continuum.

a. The motions of the individual particles cannot be mapped as affine deformations from the initial reference frame to a deformed configuration.

b. Forces between material points are not related to their relative displacements if those displacements are greater than the nominal particle diameter.

c. Interparticle forces do not depend on relative displacement rates. The non-viscous nature of flowing soil gives rise to multi-phase behavior.

\subsection{Discrete Element Method}

An alternative to the continuum description for soil and rock mechanics problems is an approach that models the material as a collection of individual particles that interact only at interparticle contact points. The "particle model," is a generic term for the class of simulation models where the discrete representation of a physical phenomenon involves the use of interacting particles. Particle models have successfully been applied to a wide class of problems in plasma physics, astrophysics, fluid dynamics, and molecular dynamics [6], [20], [24], [31], [35], and [40]. A particle model consists of a set of particles each of which has an individual collection of attributes (e.g., mass, particle position, velocity) and some constitutive relationships describing the interaction among particles. The particle attributes evolve according to the equations of motion.

If a rate independent viscosity is considered by introducing a rate dependent viscosity such as

$$
\mathrm{T}=\mu_{o} \frac{D}{F C D}
$$

Then

$$
\mathrm{F}(\mathrm{T})=\mu_{o} \Rightarrow \text { yield stress }
$$

Cundall [1974] was one of the first to use particle-modeling techniques for evaluating soil and rock mechanics problems. He coined the term Distinct Element Method (DEM) and 
developed computer programs for evaluating two-dimensional blocks of rock with complex shapes [15]. Cundall later developed the Universal Distinct Element Code (UDEC) for evaluating two- and three-dimensional interaction of a mixture of rock blocks that have different types of deformability [16]. UDEC provided capability for dealing with joint constitutive behavior, dynamic cracking, fluid flow and fluid pressure effects. In the late 1970's Cundall developed the computer program BALL to conduct research into the behavior of assemblies of discs and spheres [14]. Several authors have used DEMs to model granular assemblies' [12], [3] $\mathrm{Ng}$ and Dobry [37] used a DEM to study small strain cyclic loading. Their simulation results agreed closely with trends found in laboratory tests of sands. Shukla and Sadd [45] used DEM to investigate how mechanical stress waves propagate in granular material and how they are influenced by media microstructure. Hopkins [25] used a variation of the DEM to model ice jamming in northern rivers and sea ice ridging in the Arctic.

Sophisticated algorithms have been developed to describe the evolution of the particulate system, including the formation and breaking of inter-element contact [48]. Determination of forces between elements requires relationships to describe normal and shear interaction at the contacts. In some models, the individual particles can even "break" when stress conditions within the particle reach some critical level. Typically, soil particles have been modeled as twodimensional circular rigid disks or three-dimensional spheres. Ting [50] has developed ellipsebased two-dimensional particles to represent the effects of contact flatness and particle angularity. Six-sided solids have also been used to model granular material [19].

The predominant disadvantage of DEMs is the enormous computational requirement for keeping track of all particle contact locations. At present, the maximum number of particles that can be feasibly handled in DEM computations is no more than a few tens of thousands of particles. Centrifuge scaling principles have been used to allow for modeling of full-scale geotechnical problems using a practical number of particles while maintaining stress similitude [48]. Of course, centrifuge scaling does not maintain geometric scaling because the ratio between structure to particle size in the model is much less than that of the actual media. $\mathrm{Ba}$ ant et al. [4] numerically found that the size of the particle simulation could influence the mode of failure. Another method to counter the computational requirement of DEM has been to couple DEM models with the finite element method (FEM) for analysis of rock mass [39], [2] and the boundary element method (BEM) for penetration tests in sand [27]. The ability to couple DEMs with FEMs or BEMs allows for the modeling of larger problems, while maintaining a minimal number of particles and reducing computer run time. However, for many problems, even this approach requires excessively large particle systems while adding the complexity of solving the coupled FEM/BEM problem.

An initial goal of this dissertation is to increase the feasibility limit of a DEM by at least a factor of ten, a value that, at best, permits study of particulate systems at scales comparable to those of laboratory testes specimens. For example, a laboratory direct shear soil test of medium sand will contain over 200,000 particles. 


\subsection{Smoothed Particle (Hydrodynamic) Codes}

A class of models that admits the flexibility in kinematics description required for particulate modeling is the smoothed particle method (SPM). The SPM was developed by Monagahan [32], [33], [34], [35] originally for astronomical computation such as for galaxy formation and cratering. More recently the method has been used for analysis of penetration problems [29]. The method consists of smoothing the governing differential equation via a convolution integral in space thereby producing a weighted average form of the equation. By operating on the integral using integration by parts, spatial derivatives are "moved" to the weighting function (which is prescribed). Thus, the differential equations are converted to integral equations in which the dependent variable does not appear in any derivative term. The time evolution of the dependant variable proceeds in a manner similar to the DEM. Ostensibly, differentiability of the dependent variable is no longer a requirement because no derivatives are computed. Thus, discontinuities, separations, and change of phase can occur without introducing significant numerical difficulties. However, it should always be kept in mind that the constitutive Equations used to derive the differential Equations that serve as a starting point of the SPM are based on the notion of affine mapping between subsequent configurations. Thus, the discontinuous deformations simulated by the method may not be consistent with the physics on which the models are based. For example, a projectile cannot pierce an (ideal) elastic-plastic material because nothing in elastic-plastic theory allows such discontinuities to form in the continuum. The formation of the hole is strictly a numerical artifact of the spreading of the particles. The SPM will provide the key concepts used for the model proposed in later chapters.

\subsection{Objective of Research}

The objective of this research is to develop an alternative approach from which a mathematical theory for the mechanics of particulate matter can be formulated. This approach will be called a smooth soil particle system. Each smoothed soil particle will be modeled essentially as a point in the soil mass at which the state of the system is monitored. The particle carries with it an estimate of the state of the soil mass within its vicinity. The objective of the theoretical development is to derive the relationships that describe the interactions among the observation points. The key feature of the theory is that as the system is refined by adding more monitoring points the equations will begin to describe the behavior of a true particulate system. Importantly, the computational implementation is similar to that of traditional DEM one-to-one models so that computer technology developed for DEM can be incorporated into the smoothed soil particle system.

The research is limited to dry, fairly uniform cohesionless soils. Specific objectives of the research can be listed as follows:

a. To develop a three-dimensional discrete elements model and improve its computational efficiency to allow for large simulations. There are two issues driving this effort. First, regardless of the "smoothing" scheme ultimately developed, it is 
likely that the general integration scheme will remain unchanged. The number of aggregate particles needed to model real problems in three dimensions will undoubtedly be large. Since, realistic problems will involve particle systems on the order of 100,000 to $1,000,000$ particles; the need for improved computational efficiency is paramount. The second need for a large particle model is to develop an ability to model laboratory experiments on a one-to-one basis. The principal requirement of the averaged particle model is that it converges to the behavior of the one-to-one model, as the analysis mesh is refined. Therefore, for the averaged model to be useful, the one-to-one model must provide an accurate representation of the real particulate medium. To provide the link between the particle model representation and actual soil behavior, particle models must, at a minimum, be able to model small, simple experiments on soil.

b. To demonstrate that simulation of laboratory experiments yields results comparable to real soils. Further, the behavior of these simulations should be controlled by welldefined material parameters. This objective must be met for discrete particle modeling to be applicable to real soils. This objective is important because the DEM makes simplistic assumptions as to the geometry and interaction of individual particles. Little work has been done to determine what properties of the DEM are required to realistically model soils.

c. To establish an averaging scheme to convert properties local to the particles (e.g. mass, momentum) into continuum attributes (e.g. density, velocity gradients). An important issue to be resolved is how the coarseness of the particle system effects the properties of the averaged continuum. It is postulated that beyond a certain threshold number of particles, the actual number of particles participating in the average has little effect on the average. This principle, if verified, would put the averaging scheme on a logically sound, yet practical basis.

d. To devise a computational procedure for modeling prototype-scale behavior using sparse smooth particle systems for which computations can be performed on existing computer systems.

\subsection{Dissertation Organization}

This Dissertation consists of seven chapters and an appendix. Chapter One covers the background and introduction of the dissertation, a review of particle modeling schemes, objective of the research, and the organization of the dissertation. The second chapter describes the development of the DEM model and efforts to improve the efficiency of the model that serves as a "numerical laboratory". The third chapter takes up the general question of descriptive averaging of physical quantities of the particulate system. It also describes the development of continuum relationships derived based on "averaged" quantities. Chapter Four describes various laboratory experiments that were performed and the comparison of these test results with results from soil particle model simulations of the laboratory tests. Chapter Five describes the development of the numerical method used in the smooth particulate model. 
Chapter Six describes the results from numerical simulations from the smoothed particle of laboratory soil experiments. Conclusions of the research and recommendations for further study are provided in Chapter Seven. 


\section{CHAPTER II}

\section{The Soil Particle Model}

This chapter describes the basic mathematical formulations used to develop the soil particle model and the effort to optimize the model for use on high performance computing resources. Examples such as soil plowing and trap door simulations are shown in Figure 2.1. The primary objective of this study was to develop a model that could simulate laboratory soil experiments in which the number of computer particles is approximately the same as the number of actual soil particles in a laboratory experiment.

By performing large particle simulations, large data sets of discrete particle information are obtained. These data are imperative to development of the smooth particle system. The principal requirement of the smooth particle model is that its results converge to the behavior of the one-to-one model, as the analysis mesh is refined. Therefore, for the averaged model to be useful, the one-to-one model must provide an accurate representation of the real particulate medium. To provide the link between the particle model representation and actual soil behavior, particle models must, at a minimum, be able to model simple experiments on soil.

The objective was not to build the most physically realistic DEM model, but to build a model that allows for large simulations from which meaningful statistical data on the particulate mass can be obtained. Simplistic assumptions were made as to the geometry and interaction of individual particles, partly on the belief that only certain details of the inter-particle interaction actually cause identifiable effects in the emergent behavior of large particle groups. The simple particle model used in the present research contains these essential physical features.

The developed model uses three-dimensional rigid spherical particles. Interparticle interactions are modeled by linear springs in the normal and tangential direction the particle contacts. A local hysteretic damping law was developed to dissipate energy. To simplify the model, particle rotation was not allowed. It is believed that for many large deformation problems (e.g. plowing) with a large number of particles, individual particle rotation is not significant to the overall kinematics. The physical interpretation of the non-rotating particle assumption is that the particles possess infinite rotational inertia. Simulations by Ting, et al. [46] show that the effect of large rotational inertia is increased internal friction of the particulate media; and, they suggest that modeling real soil strength behavior requires that the rotational inertia of particles be increased significantly. Chapter four will show comparisons of computer simulations and laboratory soil tests and draws conclusions on when particle rotation is important. 

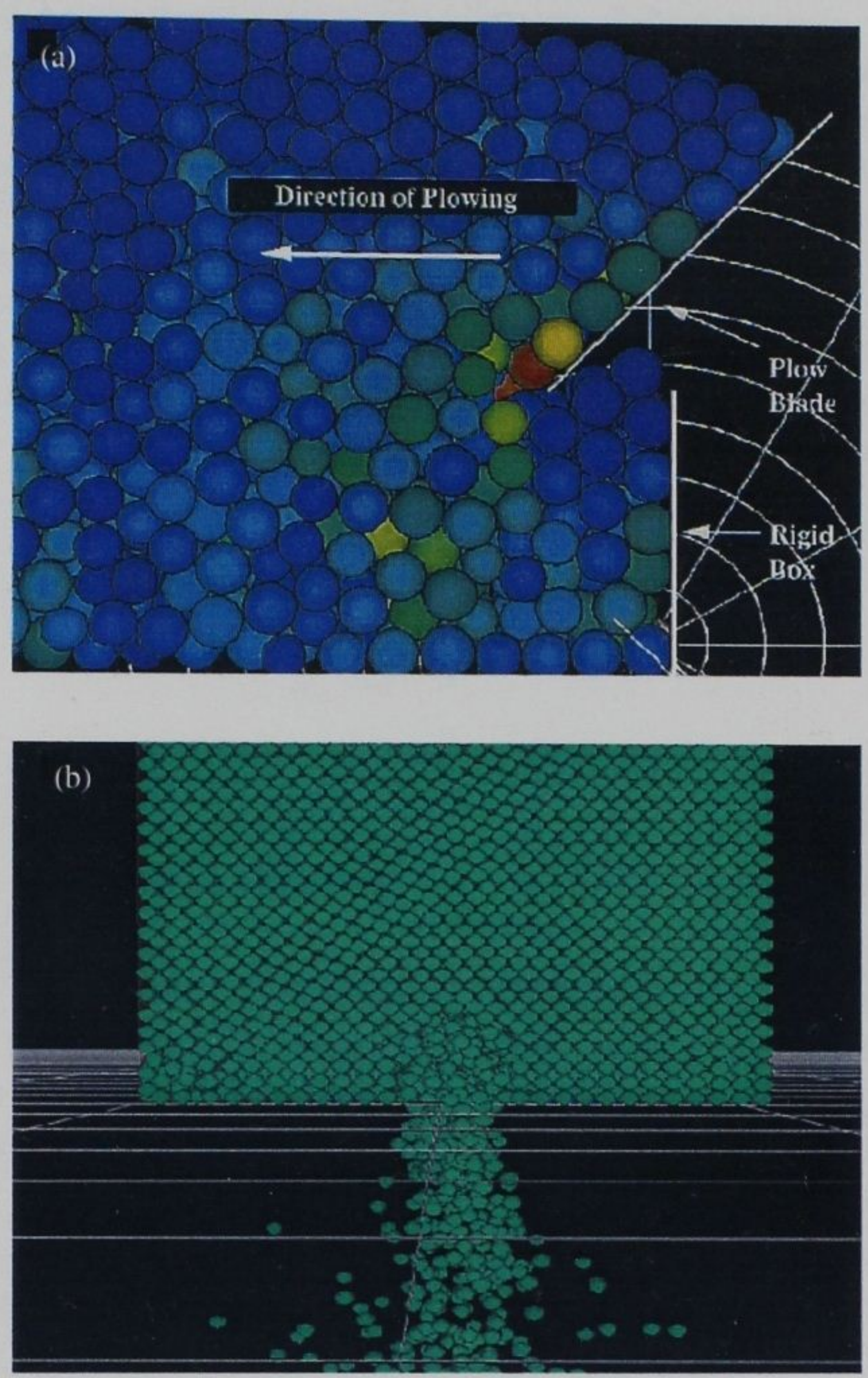

Figure 2.1: Examples of DEM (a) Soil Plowing and (b) Trap Door Simulations 


\subsection{Stability of DEM}

Discrete element models generally use explicit integration schemes to determine the motion of the particles. This is because particle contacts are formed and lost during a time step leading to highly discontinuous non-linear behavior.

Implicit integration schemes require difficult non-linear iteration, which involves the solution of very large systems of equations. Explicit integration schemes avoids iterations but their singularity comes at the expense of limitations in the computational time step. This section considers this limitation in detail. The critical time-step, $t_{c r}$, required to ensure that the numerical scheme will remain stable during the simulation is approximated from a single degree-of-freedom system as:

$$
t_{c r}=2 \sqrt{\frac{m}{k}}
$$

where $m$ is the mass of the smallest particle and $k$ is the contact spring stiffness of the smallest particle. In practice, the approximate critical time step is reduced by at least a factor of ten to ensure stability of the simulation [14].

Corkum and Ting [13] stated that if two particles are in contact for less than three time steps, energy produced from the contact is not necessarily conserved. However, if the contact lasts for three time steps or more, energy is conserved. Corkum and Ting derived relationships (Equations 2.2 and 2.6) to ensure that the contact existed for at least three time steps for particle-wall interaction and particle-particle interaction. For particle wall-interaction, the stability criteria is:

$$
2-\frac{k t^{2}}{m}-\frac{c t}{m} \geq 0
$$

where $c$ is a viscous damping coefficient.

$A$ line on a plot of $\pi_{k}$ versus $\pi_{c}$ can be defined that separates stable and unstable simulations:

$$
2-\pi_{k}-\pi_{c}=0
$$

where $\pi_{k}$ and $\pi_{c}$ are dimensionless terms defined as (see appendix A):

and

$$
\pi_{k}=\frac{k t^{2}}{m}
$$




$$
\pi_{c}=\frac{c t}{m}
$$

$A$ similar relationship can be developed for particle-particle interactions. The stability criteria for particle-particle interactions with no global damping is:

$$
2-\frac{2 k t^{2}}{m}-\frac{2 c t}{m} \geq 0
$$

or

$$
2-2 \pi_{k}-2 \pi_{c}=0
$$

or

$$
\pi_{k}=1-\pi_{c}
$$

The advantage of using these critical time step relationships is that the effect of the viscous damper is included in the calculation and provides a more accurate estimate of the true critical time step for the system. The viscous damper, as well as any other damping mechanism, stiffens the particulate system and reduces the critical time step. Equation (2.2) and (2.6) illustrate how the viscous damper stiffens the system and lowers the critical time step of a system.

The issue of a critical time step is very important when trying to model real granular material. Sands consist of particles that have relatively small mass and large contact stiffness. This combination can drive the critical time step to such a small number that meaningful simulations cannot be performed. The dimensional analysis was originally performed (Appendix A) to determine if scaling laws could be used to increase the critical time step while maintaining stress similitude. The results indicate that scaling laws do not provide any advantage to modeling the system. In fact, it was found that to maintain the stress-strain relationship for the material, the particle spring stiffness is dependent on the size of the particle. The smaller the particle is, the smaller the particle stiffness must become to maintain the stressstrain relationship of the material. Additionally, the critical time step was found to be proportional to the particle size. As smaller particle sizes are used, for a given material, the critical time step will decrease. This indicates that DEMs have a computational limit with respect to modeling small soil grains.

\subsection{Particle Force Calculations}

The interaction between two particles can be described by a set of springs, dashpots, and a frictional slider as shown in Figure 2.2. Normal and shear forces are computed between the two particles if the two particles are in contact. Two particles are in contact only if the distance, $\mathrm{D}$, between their centers is less than or equal to the sum of their radii, i.e.: 


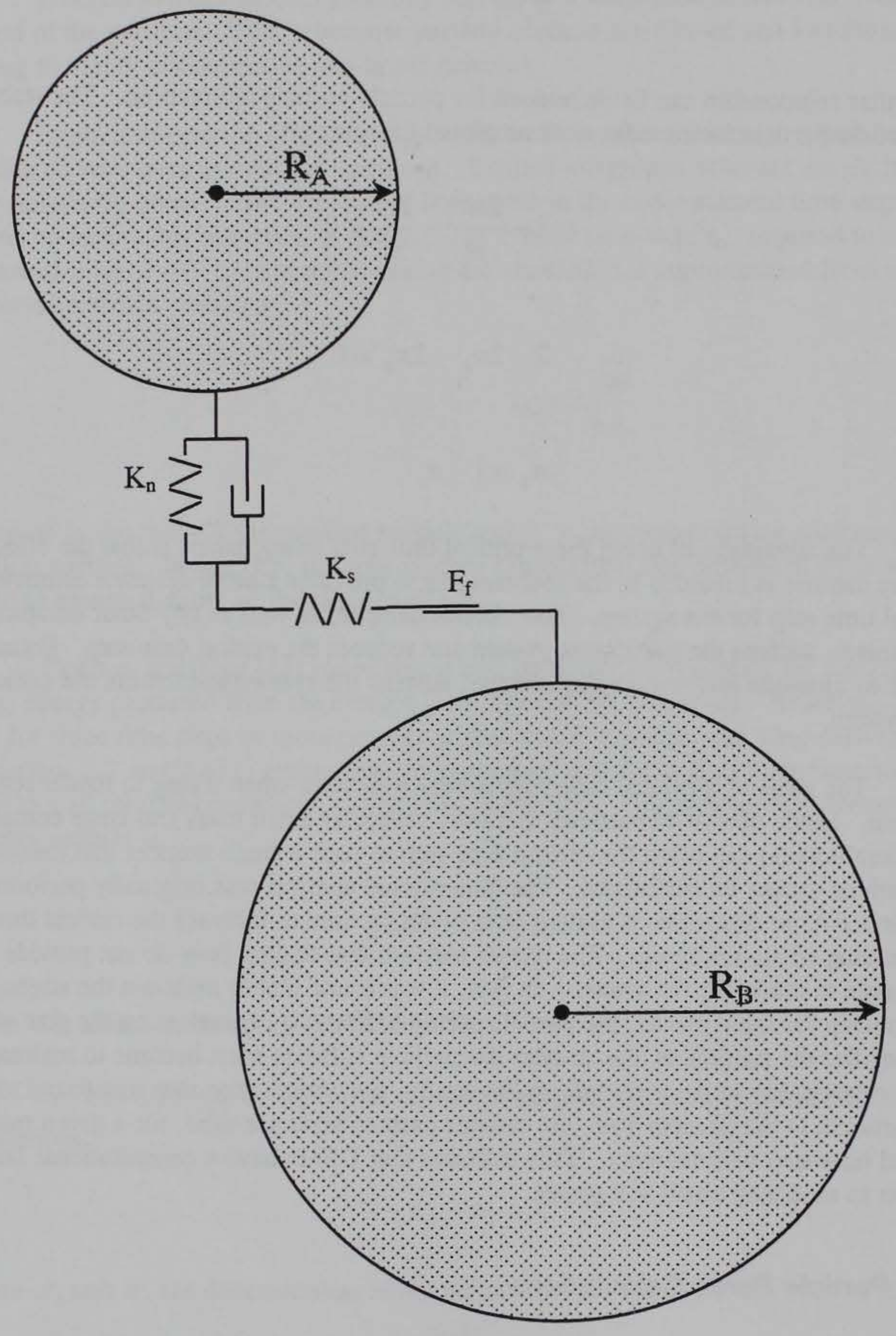

Figure 2.2: Particle Contact Interaction Model 


$$
D \leq R_{A}+R_{B}
$$

where $R_{A}$ and $R_{B}$ are the radii for particles $\mathrm{A}$ and $\mathrm{B}$.

If two particles are in contact, the normal unit vector, $n_{i}$, and the shear unit vector $t_{i}$, are determined. The normal unit vector $n_{i}$, points from the center of one particle $\mathrm{A}$ to the center of the particle B:

$$
n_{i}=\frac{B_{i}-A_{i}}{D}
$$

where $A_{i}$ and $B_{i}$ are the center point locations for particles $\mathrm{A}$ and $\mathrm{B}$ and the subscript $i$ represents the coordinate direction.

The shear vector $t_{i}$, is computed from the relative shearing velocity between the two contacting particles and is determined by:

$$
t_{i}=\frac{v_{i}^{s}}{\left|v^{s}\right|}
$$

and

$$
v_{i}^{s}=v_{i}^{r}-v_{i}^{n}
$$

where $v^{s}$ is the relative shear velocity component of the two contacting particles, $v^{n}$ is the relative normal velocity component of the two contacting particles, and $v^{r}$ is the relative velocity of the two particles in contact, given by

$$
v_{i}^{r}=v_{i}^{A}-v_{i}^{B}
$$

Such that

$$
v_{i}^{n}=\left(v^{i} \cdot n\right) n_{i} .
$$

\subsubsection{Normal Force}

The normal force between the two particles, which is a repulsive force acting along the normal unit vector, is computed as: 


$$
f_{(N)}^{A B}=\Psi K_{(N)}^{A B}
$$

where $K_{(N) \text { eff }}^{A B}$ is the normal effective stiffness constant for the two particles defined as:

$$
K_{(N) e f f}^{A B}=\frac{1}{\frac{1}{K_{N}^{A}}+\frac{1}{K_{N}^{B}}} .
$$

$K_{N}^{A}$ and $K_{N}^{B}$ are the normal stiffness constants for particles A and B, respectively, and $\Psi$ is a penalty function that reduces particle penetrations. Because the stiffness of a particle is dependent on the particle size, $K_{N}^{A}$ will not equal $K_{N}^{B}$ when the contacting particles are of different sizes. A simple linear contact stiffness is used because it is believed that for large strain problems the nature of the force-displacement relationship at contacts is relatively unimportant. Rather, most deformation occurs due to interparticle movement's [17]. The penalty function was developed with two conditions in mind. First, $\Psi$ should equal zero when $D-\left(R_{A}+R_{B}\right) \geq 0$. Secondly, if $D-\left(R_{A}+R_{B}\right)<0$, then as the distance between particle centers becomes smaller, $\Psi$ should increase.

\subsubsection{Shear Forces}

The shear force component of the particle-particle interaction is computed by integrating the relative shear velocity, $v_{S}^{A B}$ over time to provide the contact shear displacement, $\delta_{S}^{A B}$, for the current time step, $\Delta t$ : :

$$
\delta_{S}^{A B}=v_{S}^{A B} \Delta t
$$

$A$ trial shear force increment for the time step is then computed as

$$
\Delta f_{(S)}^{A B}=K_{(S)_{e f f}}^{A B} \delta_{S}^{A B}
$$

where $K_{(s) \text { eff }}^{A B}$ is the effective shear spring stiffness constant.

As long as the two particles remain in contact, the shear force increment is added to the previously accumulated shear force for the contact, i.e.,

$$
f_{(S) L}^{A B}=f_{(S) L-1}^{A B}+\Delta f_{(S)}^{A B}
$$


where the indices $L$ and $L-1$ refer to times $t_{L}$ and $t_{(L-1)}$, and $\Delta t=t_{L}-t_{(L-1)}$.

The magnitude of the trial shear force is compared to a maximum shear force computed by a Coulomb-type friction law given by Equation 2.20. If the trial shear force exceeds this maximum shear force; the shear force is set equal to the maximum shear force:

$$
f_{(S)}^{A B} \leq f_{(N)}^{A B} \tan \phi_{e f f}^{A B}
$$

where $\phi_{\text {eff }}^{A B}$ is the contact friction angle.

\subsection{Energy Damping}

The energy dissipation characteristics of the contact interaction law are critical for realistic simulation [14]. The major simplifying assumption that makes DEM computationally viable is that the particles are rigid and that interaction between particles occurs at the contact through discrete contact mechanisms. In real soil grains, energy is dissipated in the complex deformation process, which may include heat generated by plastic deformation, abrasion of the particle contact area, creation of sound and chipping or splitting of the particle. Particle interaction with boundaries may involve energy dissipation due to energy carried through the boundary materials. All of these mechanisms must be captured by the energy dissipation characteristics of the simple contact mechanisms. To improve energy dissipation characteristics, the introduction of a global damper was suggested by Cundall and Strack [14]. The global damper invokes a viscous force on a particle in proportion to its velocity, as if the particles were moving in a viscous fluid. Of course, for most simulations, the particles are not moving in a viscous fluid, leading to physically erroneous effects.

In many problems, the static position of the particulate mass is of interest. In the static position, DEM represents a spring-mass (elastic) system with constant interparticle connectivity that, in the absence of energy dissipation will oscillate freely at its resonant frequency about the equilibrium position at amplitude on the order of the particle size. In real granular media the numerous mechanisms that exist to dissipate energy, not modeled by the DEM, ensure such extreme oscillations do not occur. Thus, a DEM must allow for the dissipation of energy to ensure that a static equilibrium position can be reached. The problem that immediately arises from integrating all energy dissipating mechanism into a single mechanisms is that the system either will be insufficiently damped or that other unwanted, physical effects will be introduced by the artificial energy dissipation law. The method used for energy dissipation should thus have the following criteria:

1) the model should be calibrated from an "integrated" measurement of energy dissipation (e.g. a coefficient of restitution

2) energy dissipation should be tied to particle interactions to avoid excessive dissipation during "free-fall" motions when coordination numbers (the average 
number of contacts per particle) are small, as is the case for global viscous damping schemes

3) motions should be strongly damped once a permanent contact is made to ensure static equilibrium can be obtained

4) spurious rate dependence should not be introduced, as may happen when viscous damping is used either at the contact or as a global effect

5) there must be a clear criteria for establishing the critical time step.

Typically, DEM energy dissipation mechanisms are shear friction sliders, which dissipate energy via Coulomb friction; local viscous damping of shear and normal contact motions; and global viscous damping [13]. The problem with the use of a viscous damper is that it introduces an artificial rate effect into the problems that is not present in granular material. Studies of wave propagation [45] indicate that such physically inappropriate damping can give rise to significant errors. As noted above, in the case of the global damper the problem may be more acute because not only is spurious rate dependence introduced but also the effect is applied even in the absence of particle interactions. Additionally, the viscous damper stiffens the contact system, thus lowering the critical time step required to maintain numerical stability.

\subsubsection{Hysteretic Contact Law}

Shukla and Sadd [45] investigated three contact laws for DEM simulations; linear, nonlinear, and nonlinear hysteretic contact laws, and demonstrated how these laws effected the wave propagation process within a dry granular media. The linear and non-linear contact laws included viscous damping while the non-linear hysteretic contact law did not. These simulations where compared against experimental tests of disks made of aluminum material. Shukla and Sadd concluded that the non-linear hysteretic damping provided the necessary damping to control the inter-granular wave amplitude behavior and was the best match with the experimental data for both the wave speed and amplitude attenuation.

In an attempt to improve the energy damping of the large particle system in the present research effort, a non-linear hysteretic damping law was developed and incorporated into the model. The hysteretic contact law is defined as a two-part curve consisting of a linear portion for initial loading and a non-linear curve for unloading and subsequent reloading. The particle force associated with the non-linear curve is computed as the product of the contact displacement, $\delta$, and a modified contact spring stiffness, $K_{T}$. The function describing $K_{T}$ was arbitrarily selected as a power function that was related to the maximum contact displacement, $\delta_{m}$, and the coefficient of restitution, $e$ :

and

$$
f=k_{T} \delta
$$




$$
k_{T}=k\left(\frac{\delta}{\delta_{m}}\right)^{n-1}
$$

where

$$
n= \begin{cases}1 & \text { if } \delta=\delta_{m} \\ \frac{2-e}{e} & \text { otherwise }\end{cases}
$$

Figure 2.3 shows a typical linear loading curve and the subsequent non-linear curve for various levels of damping. The coefficient of restitution is defined as the height to which a particle will bounce after being dropped onto a rigid, flat surface divided by the initial drop height of the particle. The power $n$ is related to the coefficient of restitution by considering that $e$ is defined as:

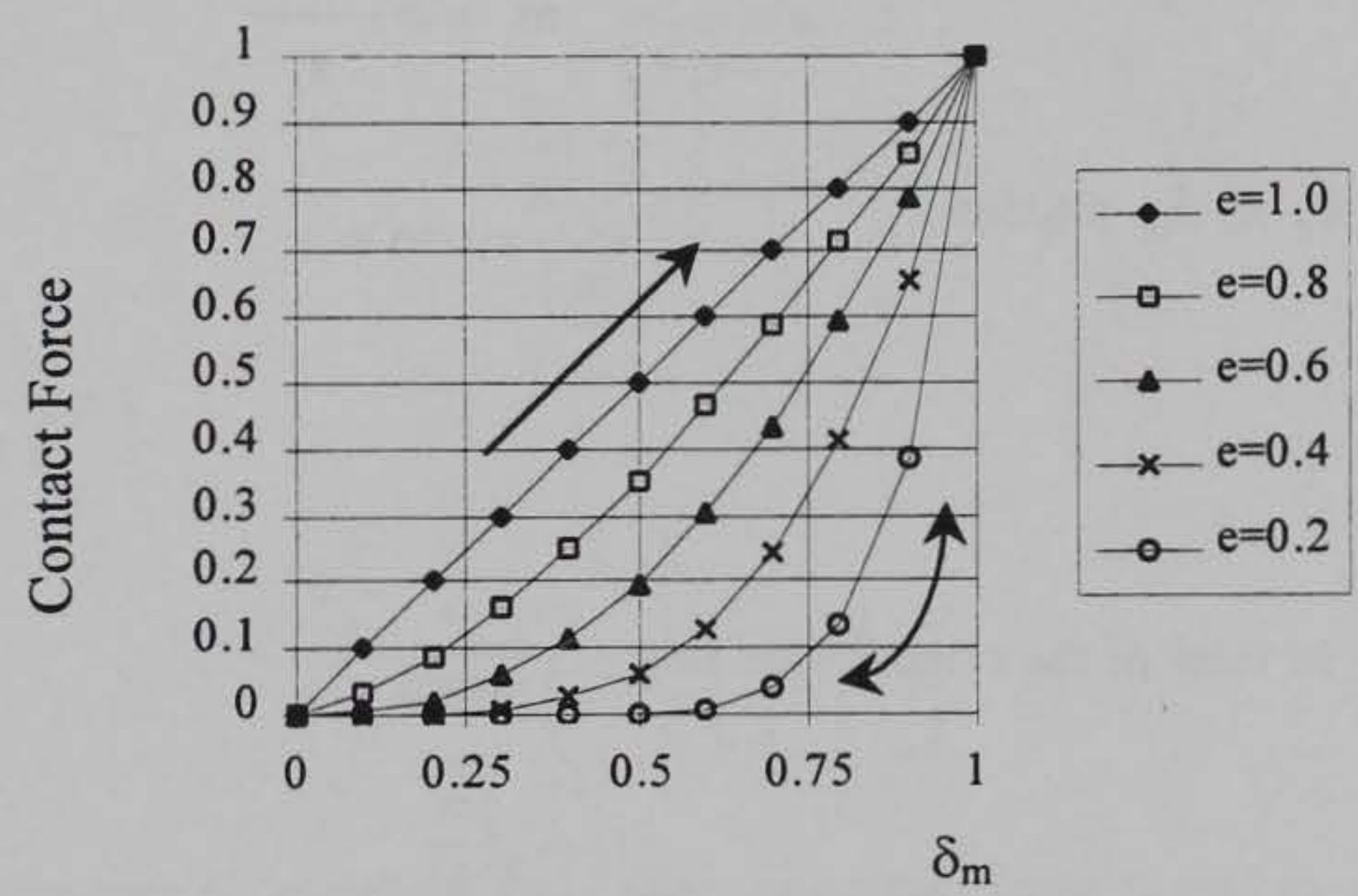

Contact Displacement

Figure 2.3: Hysteretic Contact Law 


$$
e=\frac{W^{o}}{W^{I}}
$$

where $W^{I}$ is the work associated with the contact during the initial loading. $W^{I}$ is defined by the initial linear loading force curve as:

$$
W^{I}=\frac{1}{2} k \delta_{m}^{2}
$$

$W^{O}$ is the work associated with the particle breaking the contact. $W^{O}$ is defined by the nonlinear curve as:

$$
W^{O}=\frac{k}{\delta_{m}^{n-1}} \int_{O}^{o n} \delta^{n} d \delta=\frac{k \delta_{m}^{n+1}}{(n+1) \delta_{m}^{n-1}}=\frac{k \delta_{m}^{2}}{n+1}
$$

Substituting Equations (2.24) and (2.25) into Equation (2.23) yields a relationship between the coefficient of restitution and $n$ :

$$
e=\frac{2}{n+1} \text { or } n=\frac{2-e}{e}
$$

If $k_{T}$ at $\delta_{m}$ satisfies

$$
t=\sqrt{\frac{m}{n k^{\prime}}}
$$

an in view of the critical time step

$$
t_{c}=\sqrt{\frac{m}{k^{\prime}}}
$$

then

$$
\frac{t}{t_{c}}=\sqrt{\frac{1}{n}}=\sqrt{\frac{e}{2-e}}
$$

Equation (2.29) can be used to evaluate how a change in $e$ will affect the critical time step. 


\subsubsection{Variable Viscous Local Damper}

The hysteretic damping law will remove energy from the system only if contacts are formed and broken. If two particles remain in contact, they will oscillate along the non-linear portion of the hysteretic curve. Thus, an additional mechanism is required to ensure that the interacting particles will come to rest. The option of creating a more general hysteretic law is rejected to avoid introducing a large set of interval variables. Therefore, a variable viscous local damper is applied to the non-linear portion of the hysteretic damping curve. The viscous damper is set so that the maximum amount of viscous damping can occur without a decrease in the critical time step. If Equation (2.6) is used as the stability criteria, then

$$
m-k_{T} t^{2}-c t=0
$$

Solving for the $c$ that just meets this criterion:

$$
c=\frac{m-k_{T} t^{2}}{t}
$$

Substituting Equations (2.22) and (2.27) into Equation (2.31) produces:

$$
c=m \frac{1-\frac{1}{n}\left(\frac{\delta}{\delta_{m}}\right)^{n-1}}{\sqrt{\frac{m}{n k}}}
$$

or

$$
c=\sqrt{n k m}\left(1-\frac{1}{n}\left(\frac{\delta}{\delta_{m}}\right)^{n-1}\right)
$$

Note that the viscous damper is "engaged" only after the contact force begins to oscillate and make a minor contribution to the total energy dispersion in the system.

\subsection{Particle Motion}

Once all of the contact forces acting on a particle have been determined for each particle, the forces are resolved into orthogonal components. A gravitational force is applied to each particle. The magnitude of the gravitational force is equal to the product of the mass of the particle and the gravitational acceleration constant for the system. Forces acting upon each particle are vectors summed and the instantaneous acceleration of the particle, over the current time step, is determined using Newton's second law of motion: 


$$
\ddot{X}_{i}^{A}=\frac{\sum F_{i}^{A}}{m^{A}}
$$

Finally, the updated velocity and location of each particle are determined using equations of motion:

$$
\dot{X}_{i, N}^{A}=\dot{X}_{i,(N-1)}^{A}+\Delta t \ddot{X}_{i,(N-1)}^{A}
$$

and

$$
X_{i, N}^{A}=x_{i,(N-1)}^{A}+\Delta t \dot{X}_{i(N-1)}^{A}+\frac{1}{2} \Delta t^{2} \ddot{X}_{i,(N-1)}^{A}
$$

\subsection{Optimization of Particle Code}

This section describes the effort to optimize the model for use with single processor computing resources. Efforts to optimize the model for parallel computing resources are described in [8]. The preliminary modeling effort of the soil particle model focused on the implementation of the DEM algorithm. Little or no attention was given to computational performance or efficiency. The algorithm consisted of two main sections: calculation of forces and integration of equations of motion to move particles. Each section loops over the entire number of particles, performing all calculations for that section on a particle-by-particle basis. Contacts are determined by calculating distances between all particles, and redundant contact and force calculations were eliminated by identifying each particle by a unique "id" number and limiting the checks to the current particle and those with a larger particle "id" number. The determination of particle contacts consumed the bulk of the computations. The resulting model, though simple, could only reasonably run problems in the couple of thousand-particle range for any simulation requiring a large number of time steps.

The initial performance evaluation concentrated on single processor performance on a CRAY Y-MP and was aimed solely at code structure. With no major algorithm modifications being made, the emphasis was on improved vectorization, improved input/output, and the elimination of redundant work. Though performance was improved by at least an order of magnitude, it was still insufficient.

Performance profiling had clearly established the contact checks as the primary computational bottleneck. The all-against-all particle distance check, though simple, scales computationally as the square of the number of particles $\mathrm{O}\left(\mathrm{N}^{2}\right)$, making it impractical for large problems. By using a link-cell type method [23], [24] to create a neighbors list [53], the contact check was reduced to an $\mathrm{O}(\mathrm{N})$ operation, dramatically reducing the computational requirement. This method divides the physical space of the simulation into a regular grid of cells. 
A particle's "owning" cell can be determined with one pass through the particles. With the cell size being set greater than or equal to the maximum particle diameter, possible contacts are limited to particles within a cell and the 26 surrounding cells. The actual implementation only puts particles into the upper (or lower) 13 surrounding cells to avoid duplicating redundant (equal but opposite) force calculations. Larger cell sizes can decrease the frequency with which one has to update the neighbor's list, but add considerably to the number of potential contacts. Since the neighbors list algorithm amounted to only $5 \%-15 \%$ of the total runtime, it was found to be more efficient to minimize the cell size and update each time step. The cell size was set to just larger than the diameter of the largest particle in the system. This ensured that the number of potential contacts within a cell was minimized and that a particle's interaction was limited to the cell it occupied and the surrounding 26 cells. Updating each time step also simplifies the transition to a parallel model.

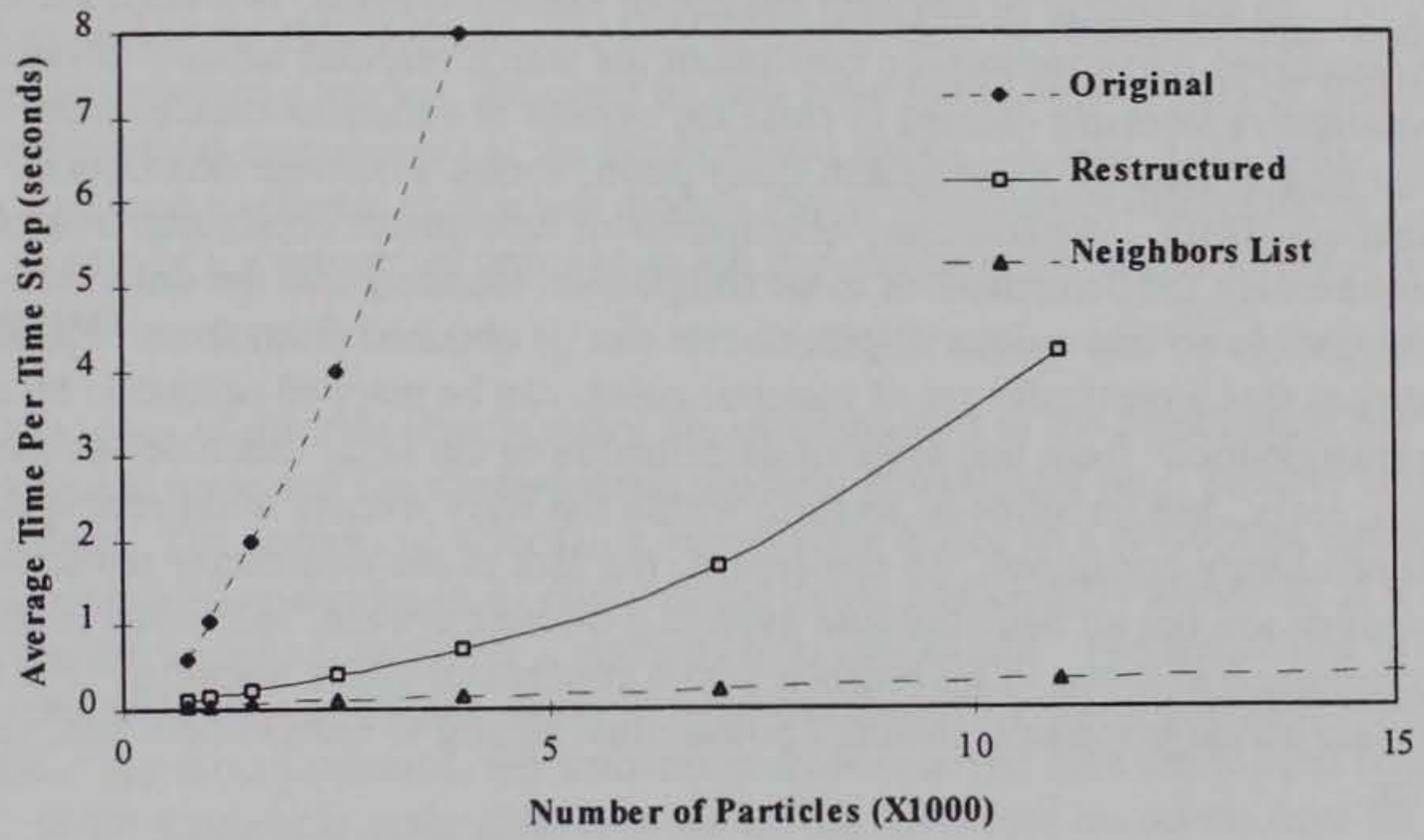

Figure 2.4: Single processor CRAY Y-MP CPU time-per-time step vs. number of particles for the three phases of single processor optimization

Figure 2.4 shows the single processor improvements for the three versions of the model on a CRAY Y-MP as the number of particle increases. The times given are an average timeper-time step. 


\section{Chapter III}

\section{Continuum Representation of Discrete Particle Systems}

\subsection{Background}

Most numerical models are discrete approximations to systems of differential equations. The differential equations are expressed in terms of continuous variables and describe the behavior of a continuous medium. In reality, all media are discrete and the continuum assumption serves only as a useful approximation that simplifies analysis. Normally, the difference in scale between the smallest element of the system and the problem domain is so great that the continuum approximation is valid.

In the course of devising models of natural systems, two steps are essential: 1) a media is conceived that constitutes a continuum for which relevant balance laws can be stated and 2) constitutive laws are defined to close the system of equations created from the balance laws. The central issue of a continuum description, versus a discrete description, is the continuity of field quantities. In particular, descriptions of deformable continuum bodies begin with a requirement for deformations to be compatible, meaning that the deformation fields are integratible so that unique displacements can be obtained from them. Physically, compatibility implies that a particular set of material points can be mapped uniquely, by affine transformation, from one stage of deformation to the next. Such deformations do not admit rips, tears, and fractures or so forth within the body without additional specification of auxiliary discontinuity conditions. In this regard, the size of the elementary particles making up the medium are not an issue because even in a discrete system, individual particles can be constrained to move in accordance with a continuous affine mapping. For movements that violate affine mappings, material points must belong to independent sets, regardless of their size.

In other applications, the size of the discrete elements becomes an issue because certain field quantities display correlation lengths whereby values of a quantity at one location are related to that quantity at another. Quantities associated with a discrete element display spatially related behavior over dimensions equal to those of the element. In media consisting of discrete elements, therefore, it is sometimes expedient to forego devising a continuum description and proceed with a model of the discrete media such as described in the previous chapter. Discrete models provide a basis for a more direct link to the underlying physics but suffer from the drawback that the numerical representation of the media is significantly coarser than the actual media. This limitation must be weighed against the reality of a numerical model of a continuum which itself is an approximation of a discrete system. It can be expected that any numerical approximation will be coarser than the actual media whether based on a discrete description or a continuum representation.

Formulations in continuum mechanics are based on field quantities such as density, stress, and strain, all quantities associated with a representative elemental volume (REV). The 
REV is taken to be that volume for which a quantity can be averaged to produce a statistically stationary result. A typical example is density, which is defined as the mass contained within a sampling volume. At the smallest scale, if the sampling volume lies within a solid grain the density will be that of the solid phase. If the sampling volume lies within a pore, the density will be zero. If an average is made for a somewhat larger volume the density will vary between the solid's density and zero. As the sampling volume increases the average density will approach the material's bulk density. The volume needed to obtain a statistically correct bulk density is the REV. For the REV formulation of the continuum, each material point is assumed to possess the attributes of the REV surrounding it. While it is recognized that the REV has a finite size, a fact that may affect accuracy of the continuum model, the REV size itself is typically not an explicit part of the continuum model. There is no REV size parameter.

While it may appear that a discrete model permits a more direct link to physical phenomenon without incurring errors significantly greater than discrete numerical approximations of continuum equations, relatively few models are actually based on discrete models. The reason for this is simply the lack of recognition of coarse discrete models as viable alternatives to continuum models. Discrete models have found applications in studies of fundamental physical mechanisms at the fundamental scales [1], [10], [11], [28]. What is lacking is a general treatment of discrete models that systematically distinguishes length scales that arise as part of the numerical approximation process from the scales intrinsic to the physical system.

Two types of discrete length scales are distinguished in the formulation that follows; a smoothing length scale and the characteristic physical length scale (e.g. particle size) of the discrete system. The numerical smoothing length scale is tied to a smoothing operator that distributes conserved quantities (e.g. momentum, mass, and energy) identified at a finite set of points within the sampling area over a mathematical continuum. The smoothing operator is applied to the difference equations, derived from the discrete system, that contains the natural length scale. For most problems, the smoothing scale is larger than the natural scale.

However, as the smoothing scale is made smaller, the difference equations describing the actual discrete system are recovered.

\subsection{Smoothing Field Quantities and Strain}

There are several motivations for using a smoothing operator in lieu of a REV. First, the method provides a systematic way to derive equations in terms of continuous functions without introducing the concept of the representative elementary volume (REV) which in turn is tied to the properties of an infinitesimal element. In the REV approach, the characteristic length scales are lost. In the smoothing approach, the discrete approximation is merely coarsened. Secondly, the smoothed media approach provides a means to convert the statistical properties of the individual components, such as grain size distribution, of the discrete system to continuous properties. For example, properties of elastic elements in a discrete particle can be related to the elastic stiffness tensor. Thirdly, the smoothed continuum properties converge to the discrete media properties as the smoothing length goes to zero. 


\subsubsection{Implied Average Volume}

The use of a smoothing operator for modeling discrete systems has been demonstrated in Smoothed Particle Hydrodynamics [7], [33], [34], and [35]. This averaging approach yields an "implied averaging volume" (IAV). The averaging is implied because the definite averaging volume of the REV is replaced by a space in which weighted averages are taken. For example, the implied mass average over the entire space is given by the integral

$$
\bar{m}(x)=\int_{-\infty}^{\infty} \phi\left(x-x^{\prime}\right) m\left(x^{\prime}\right) d x^{\prime}
$$

where $x$ is the location of the averaging point and $x^{\prime}$ is a location of a particle in the vicinity of $x$. The kernel function, $\phi\left(x-x^{\prime}\right)$, weights the average with distance from the material point to which the average is assigned, giving rise to a characteristic length similar in concept to the REV dimension. The characteristic length, $h$, of the system as shown in Figure 3.1 is defined as the size at which the average value of a quantity becomes statistically stationary. The kernel function is symmetric in its argument, monotonically decays with distance, and satisfies

$$
\int_{-\infty}^{\infty} \phi\left(x-x^{\prime}\right) d x^{\prime}=1 \text {. }
$$

The integral has compact support meaning

$$
\bar{m}(x)=\int_{x-h}^{x+h} \phi\left(x-x^{\prime}\right) m\left(x^{\prime}\right) d x^{\prime}
$$

where

$$
\int_{x-h}^{x+h} \phi\left(x-x^{\prime}\right) d x^{\prime}=1
$$

and

$$
\phi\left(x-x^{\prime}\right)=0 \forall\{|x-x|>|h|\}
$$

Within the interval $\left|x-x^{\prime}\right| \leq|h|$, the kernnel $\phi\left(x-x^{\prime}\right)$ is assumed to be sufficiently smooth to permit mass distributions of the form 


$$
m(x)=\sum_{k=1}^{N} m^{k} \delta\left(x-x^{\prime}\right)
$$

where $m^{k}$ is the mass of the $k^{\text {th }}$ particle located at $x^{k}$ in the system. The Dirac delta operator, $\delta\left(x-x^{k}\right)$, satisfies Equation (3.2) and is zero for $x \neq x^{k} . N$ is the total number of particles in the system. Equation (3.6) holds for any $x$ including $x^{\prime}$. Thus by substituting Equation (3.6) into (3.1):

$$
\bar{m}(x)=\int_{x-h}^{x+h} \phi\left(x-x^{\prime}\right) \sum_{k=1}^{N} m^{k} \delta\left(x^{\prime}-x^{k}\right) d x^{\prime} .
$$

The redistribution from discrete particle masses to the continuum of material points is mass conserving for the whole domain as follows

$$
\int_{-\infty}^{\infty} \bar{m}(x) d x=\int_{-\infty}^{\infty} \int_{x-h}^{x+h} \phi\left(x-x^{\prime}\right) \sum_{k=1}^{N} m^{k} \delta\left(x^{\prime}-x^{k}\right) d x^{\prime} d x
$$

Upon changing the order of summation and integration over $x^{\prime}$ and invoking the selector property of the delta operator we get

$$
\int_{-\infty}^{\infty} \bar{m}(x) d x=\int_{-\infty}^{\infty} \sum_{k=1}^{N} m^{k} \phi\left(x-x^{k}\right) d x
$$

Again, interchanging the order of summation and integration and noting the infinite extent of the integral, we evaluate the integral using Equation (3.2) to get

$$
\int_{-\infty}^{\infty} \bar{m}(x) d x=\sum_{k=1}^{N} m^{k}
$$

The effect of mass averaging is to distribute (or numerically diffuse) the particle masses in accordance with the weighting kernel to produce a continuous field $m(x)$. Like the REV, the IAV produces a continuum in which each material point within the medium is given a property corresponding to the smoothed average for particles making up the (actual) discrete media. The key advantage of the IAV over the REV is that a characteristic size of the smoothing function can be incorporated into the continuum definition. 

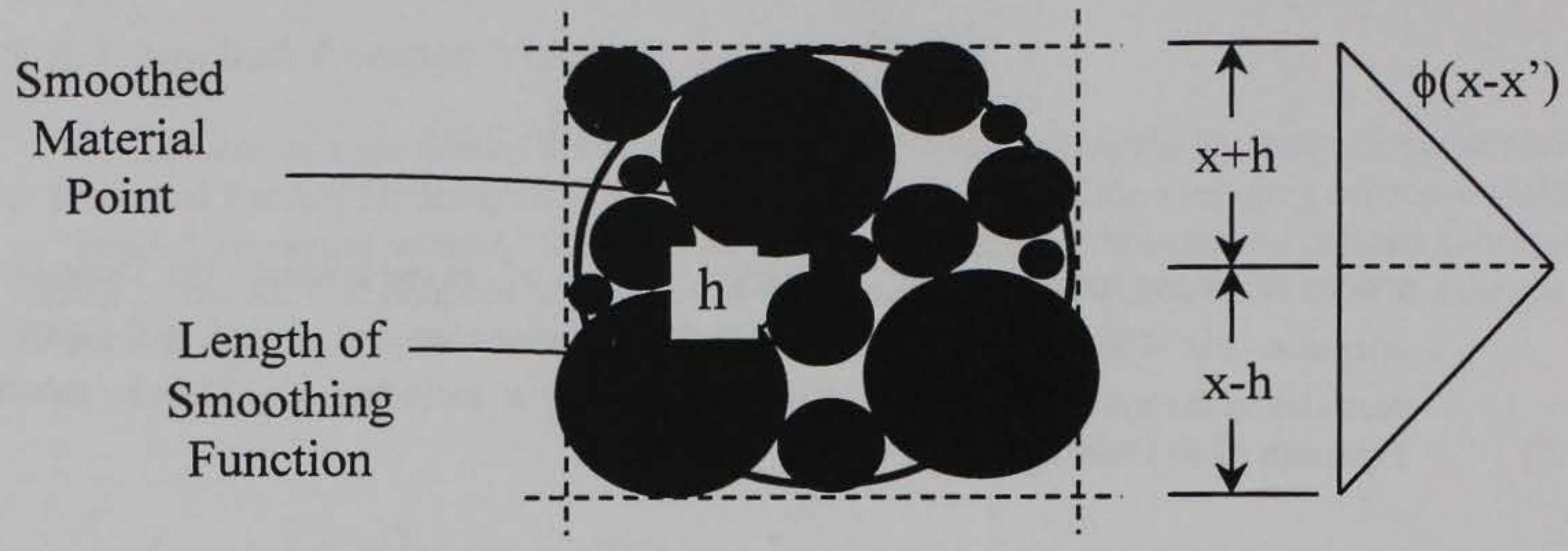

\section{Discrete Particle System}

Figure 3.1: Characteristic Length of the Kernel Function

\subsubsection{Computation of Gradients}

A major advantage of the smoothing operator, Equation (3.1), is that derivatives and gradient operators for the smoothed quantities can be computed. The average gradient of the mass is defined as

$$
\overline{\nabla m}(x)=\int_{x-h}^{x+h} \phi\left(x-x^{\prime}\right) \nabla m\left(x^{\prime}\right) d x^{\prime} .
$$

This formula has limited application in view of the character of $m(x)$ given by Equation (3.6). However, in view of the smoothness requirements imposed on $\phi(x)$, integration by parts may be applied to the integral to obtain

$$
\overline{\nabla m}(x)=\int_{x-h}^{x+h}-\nabla \phi\left(x-x^{\prime}\right) m\left(x^{\prime}\right) d x^{\prime} .
$$

Note that the boundary term resulting from the integration by parts is zero when evaluated at the limits of $\mathrm{x}+\mathrm{h}$ and $\mathrm{x}-\mathrm{h}$. Thus the gradient of the mass average becomes the weighted average of the mass using $-\nabla \phi\left(x-x^{\prime}\right)$ as the weighting kernel. The gradient is taken over the same volume of mass as the average itself. 


\subsubsection{Momentum, Velocity, and Velocity Gradient}

It is often useful to define averages in terms of weighted quantities where the weighting is in terms of a quantity, such as mass, that measures the attributes of the sampling space. A physically motivated example of a mass-weighted quantity is the average velocity, $\bar{v}_{i}(x)$, which is determined from the averaged momentum, $\bar{p}_{i}(x)$ and average mass, $\bar{m}(x)$ :

$$
\bar{v}_{i}(x)=\frac{\bar{p}_{i}(x)}{\bar{m}(x)}
$$

where

$$
\bar{p}_{i}(x)=\int_{-\infty}^{\infty} \phi\left(x-x^{\prime}\right) m\left(x^{\prime}\right) v_{i}\left(x^{\prime}\right) d x^{\prime}
$$

and $\bar{m}(x)$ is computed from Equation (3.1).

The spatial velocity gradient, $L_{i j}$, is given by

$$
L_{i j}=\nabla \bar{v}_{i}(x)=\frac{1}{\bar{m}(x)} \nabla \bar{p}_{i}(x)+\frac{1}{\bar{m}(x)^{2}} \bar{p}_{i}(x) \nabla \bar{m}(x)
$$

The second term in Equation (3.15) accounts for the non-uniform distribution of particles since $\bar{m}(x)$ varies with $x$.

\subsection{Sampling Size Effects}

One of the primary advantages of the descriptive averaging is that size effects are accounted for through the use of the characteristic length of the kernel function. An understanding of how the characteristic length or sampling size affects the descriptive averaging process is required. If the characteristic length is set too small, the results from the descriptive averaging process will not properly model the macroscopic behavior. Conversely, if the characteristic length is too large, the results from the averaging process will tend to smooth out any localized effects, such as shear banding.

An additional concern about the characteristic length is its effect on the numerical accuracy of gradients. Since gradients reflect change in a property or behavior, they require more data than a point variable, such as mass, to be reliable. To illustrate this point, the particle data set from the Treasure Island sample, described in Chapter 4, was analyzed. Each particle was assigned a horizontal velocity equal to the particle's vertical position. By 
definition, this should yield a value of one for the shear component of the spatial velocity gradient, $L_{12}$, as defined by Equation (3.15). However, some deviations from this ideal should be expected because in the averaging scheme the mass of a particle is lumped at the center of the particle. This can lead to an uneven distribution of masses that in turn will effect the estimate of the mass gradient.

The shear component of the spatial velocity gradient was sampled using four different sampling lengths for two different kernel functions. The first kernel function is a hat function shown in Figure 3.1. The second kernel is a spline function [35] used in smoothed particle hydrodynamics. Figure 3.2 demonstrates the impact that sampling size (i.e., number of particles in the sample) has on the accuracy of the estimated gradient. Clearly, a fairly large number of particles, 200 or more is needed to deliver a good estimate of the gradient for this soil.

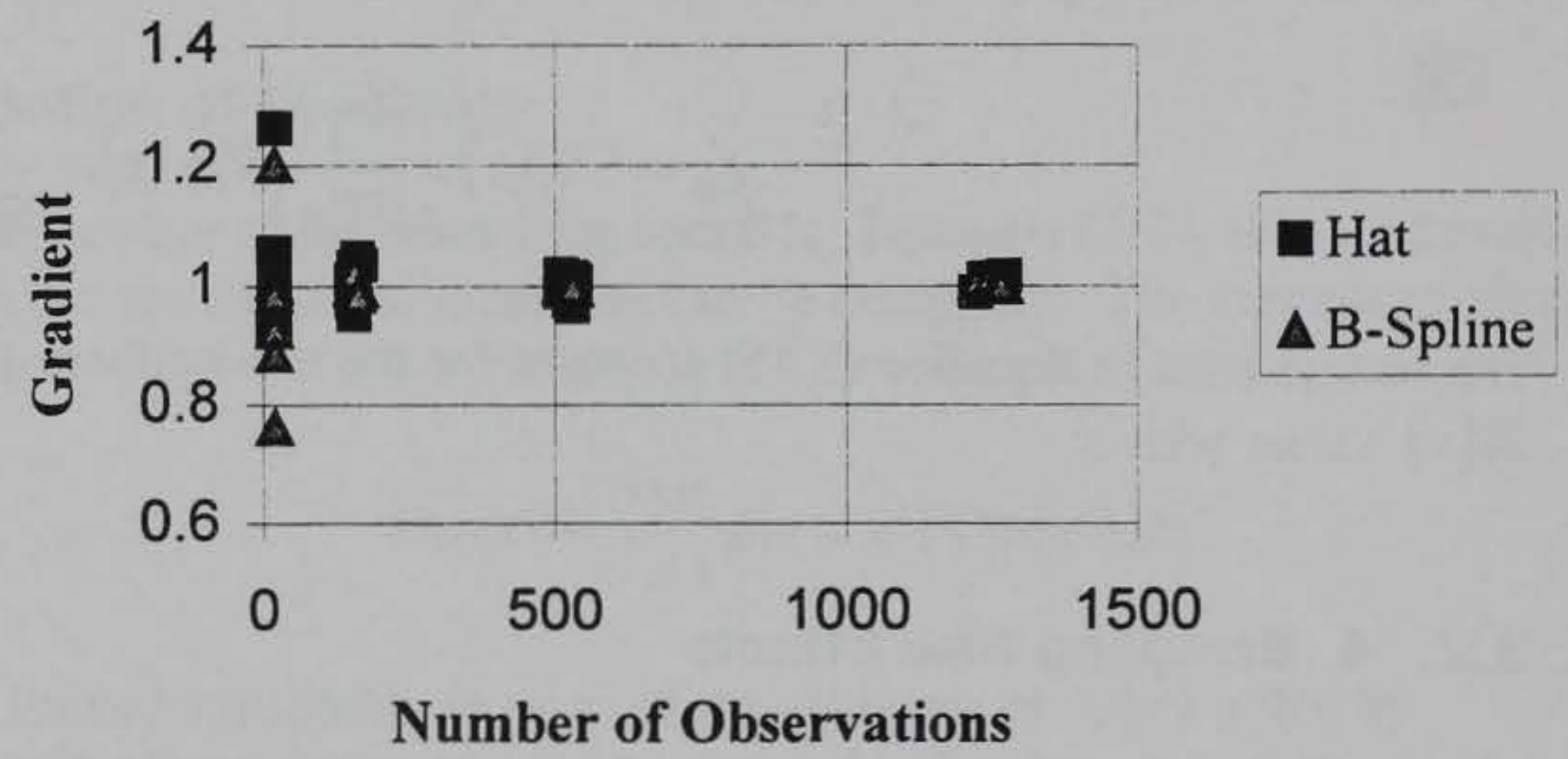

Figure 3.2: Effects of Sampling Size on Gradient Estimate

The spline kernel function provided a slightly better estimate of the gradient than the hat kernel function. However, because of the complexity of the spline kernel function and additional computational requirements for computing averages, the hat kernel function was used for the remainder of the research. 


\subsubsection{Strain}

The ability to compute the spatial velocity gradient from the discrete system allows for the measurement of strain in the system. Typically, Lagrangian strain is used to describe the straining. Lagrangian strain is measured relative to an initial condition. A transformation between the spatial coordinate grid, from where the displacement measurements are taken, and the material grid, Figure 3.3, which relates the system relative to an initial configuration, must be developed to determine the Lagrangian strain history of material points during the simulation. To relate points between the two coordinate systems a deformation gradient tensor is defined as:

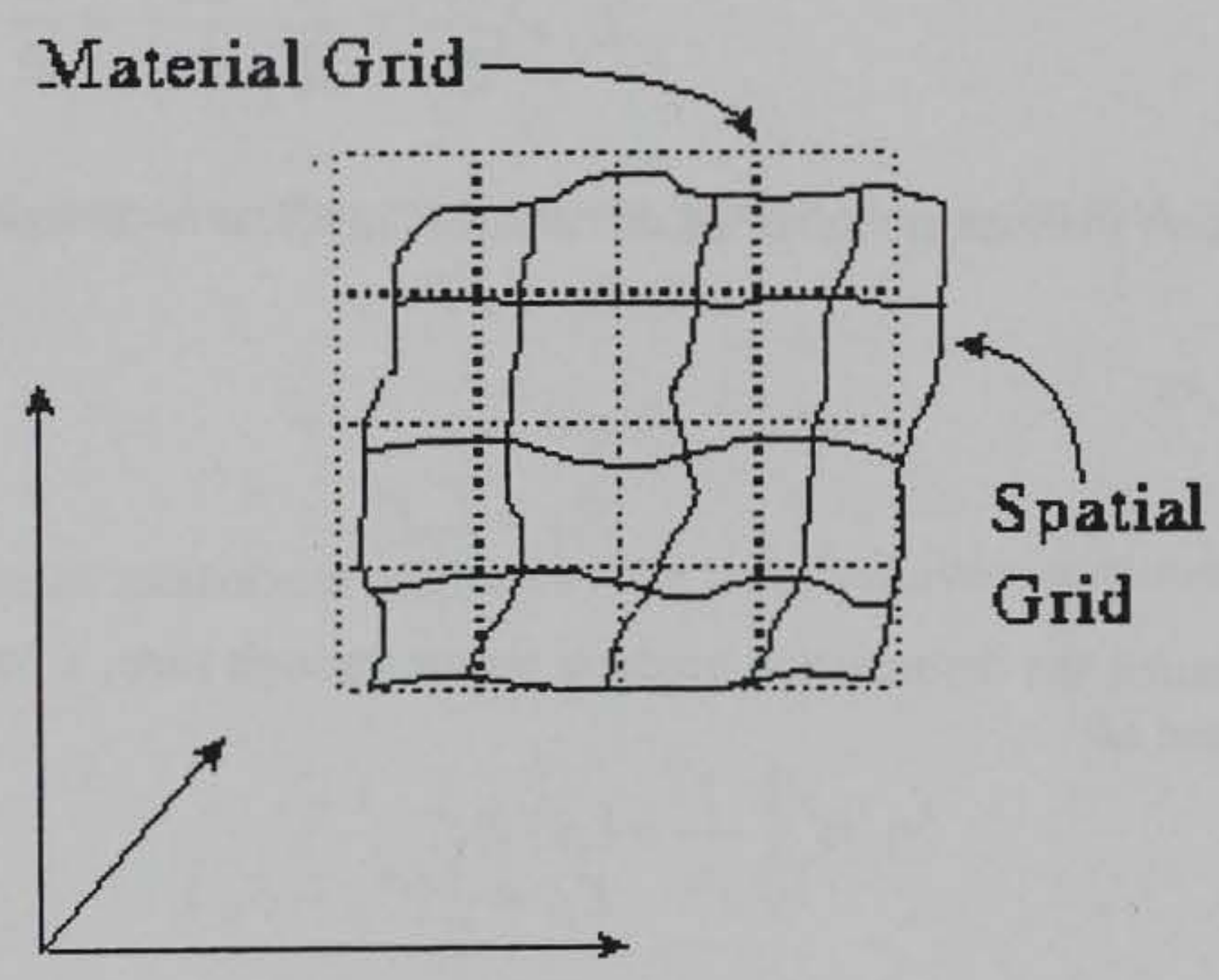

Figure 3.3: Deformed Material and Spatial Grid 


$$
F_{i J} d X_{J}=\frac{\partial x_{i}}{\partial X_{J}} d X_{J}
$$

The upper case subscripts signify derivatives are taken with respect to the material coordinates. The lower case subscript implies the direction in the spatial coordinate system, $F_{i J}$ maps from the reference grid to the spatial grid. The mapping between the spatial and reference grid must obey certain conditions. First, the axiom of impenetrability states that no two particles can occupy the same location in space at a given time. This requires that the mapping produce a single unique location for each particle in each grid system. Additionally, any two neighboring points in the reference grid must remain neighbors in the spatial grid. The mapping between the grids must be continuous and possess continuous derivatives with respect to space and time. The above conditions are met if the determinant of $\mathrm{F}$ is non-zero. This determinant is referred to as the Jacobian determinant, $\mathrm{J}$.

The deformation gradient tensor is related to the spatial velocity gradient tensor,

$$
L_{i j}=\frac{\partial v_{i}}{\partial x_{j}}=\frac{\partial v_{i}}{\partial X_{j}} \frac{\partial X_{J}}{\partial x_{j}}=\frac{d}{d t} \frac{\partial x_{i}}{\partial X_{J}} \frac{\partial X_{J}}{\partial x_{j}} .
$$

Therefore, the rate at which the deformation gradient is changing can be written as:

$$
L_{i j}=\dot{F} F^{-1}
$$

or

$$
\dot{F}_{i J}=L_{i j} F_{j J}
$$

By updating the deformation gradient tensor through time, a Lagrangian strain tensor can be computed as:

$$
E_{I J}=\frac{1}{2}\left(C_{I J}-\delta_{I J}\right)
$$

where $\delta_{I J}$ is the Kroncker delta function and $C_{I J}$ is the Green's deformation tensor defined as:

$$
C_{I J}=F_{k l} F_{k J}=F^{T} F
$$

The rate of change for the Lagrangian strain tensor is:

$$
\dot{E}_{I J}=\frac{1}{2} \frac{d}{d t}\left(F_{i I} F_{i J}-\delta_{L J}\right)
$$


or

$$
\dot{E}_{L J}=F_{i l} F_{i J} .
$$

Finally, substituting Equation (3.19) into Equation (3.23) yields:

$$
\dot{E}_{I J}=L_{i J} F_{j l} F_{i J}
$$

\subsection{Kinematics of Averaged Quantities}

At the particle level Newton's law for linear momentum gives the kinematics relationship:

$$
\sum_{c=1}^{c_{k}} f_{i}^{c}=\dot{p}_{i}
$$

where $C_{k}$ is the total number of contacts acting on particle $k$. In the case of a constant particle mass

$$
\dot{p}_{i}=m \dot{v}_{i}
$$

The smoothed average of momentum is given by the weighted average of momentum of the $K_{s}$ particles in the region:

$$
\bar{p}_{i}\left(x_{i}\right)=\frac{1}{\rho_{s}} \sum_{k=1}^{K_{s}} \phi^{k} p_{i}^{k}
$$

where

$$
\rho_{s}=\sum_{k=1}^{K_{s}} \phi^{k}
$$

and

$$
\phi^{k}=\phi\left(x_{i}-x_{i}^{k}\right)
$$

where $x_{i}^{k}$ is the coordinate of the particle center while the smoothed average momentum $\bar{p}_{i}$ is a function of the positional coordinate $x_{i}$. The average momentum rate is given as: 


$$
\dot{\bar{p}}_{i}=\frac{1}{\rho_{s}} \sum_{k=1}^{K_{s}}\left\{\dot{\phi}^{k} p_{i}^{k}+\phi^{k} \dot{p}_{i}^{K}\right.
$$

where

$$
\dot{\phi}=\frac{\partial \phi^{k}}{\partial x_{j}} \frac{\partial x_{j}^{k}}{\partial t}=\nabla_{j} \phi^{k} v_{j}^{k}
$$

It follows from Equations (3.25) and (3.27) that

$$
\dot{\bar{p}}_{i}=\frac{1}{\rho_{s}}\left\{\sum_{k=1}^{K_{s}} \nabla_{j} \phi^{k} v_{j}^{k} p_{i}^{k}+\sum_{k=1}^{K^{k}} \phi^{k} \sum_{c=1}^{C_{K}} f_{i}^{c}\right\}
$$

where

$$
\nabla_{i}=\frac{\partial}{\partial x_{i}}
$$

The first term of Equation (3.32) is the convective term that arises because the averaging is performed over a grid (possibly fixed in space) that moves at a velocity $v_{i}^{k}$ relative to the weighted average of the particles. For simplicity of presentation, an observation frame is selected such that $v_{i}^{k}=0$.

By regrouping the remaining term, the sum on the right hand side, Equation (3.32) can be written as

$$
\dot{\bar{p}}_{i}=\frac{1}{\rho_{s}} \sum_{l=1}^{L_{s}}\left(\phi^{1} f_{i}^{1}+\phi^{2} f_{i}^{2}\right)^{l}
$$

where the sum is taken over $\mathrm{L}_{\mathrm{s}}$ particle contacts within the sampling region. The superscripts 1 and 2 denote the two particles making up the $l$-th contact as shown in Figure 3.4.

Regardless of which particle is labeled 1 or 2 we have

$$
n_{i}^{1}=n_{i}^{2}
$$

and

$$
f_{i}^{1}=-f_{i}^{2}
$$


It follows that,

$$
f_{i}^{1} n_{j}^{1}=f_{i}^{2} n_{j}^{2}
$$

and

$$
f_{i}^{1} n_{i}^{1}=f_{i}^{2} n_{i}^{2}
$$

It is of interest to relate the right hand side of Equation (3.34) to a gradient of stress with the goal of obtaining a relationship comparable to the conservation of linear momentum for a continuum. To this end, the particle stress is defined as:

$$
\sigma_{i j} \equiv \frac{R}{V} \sum_{c=1}^{c} n_{i}^{c} f_{j}^{c}
$$

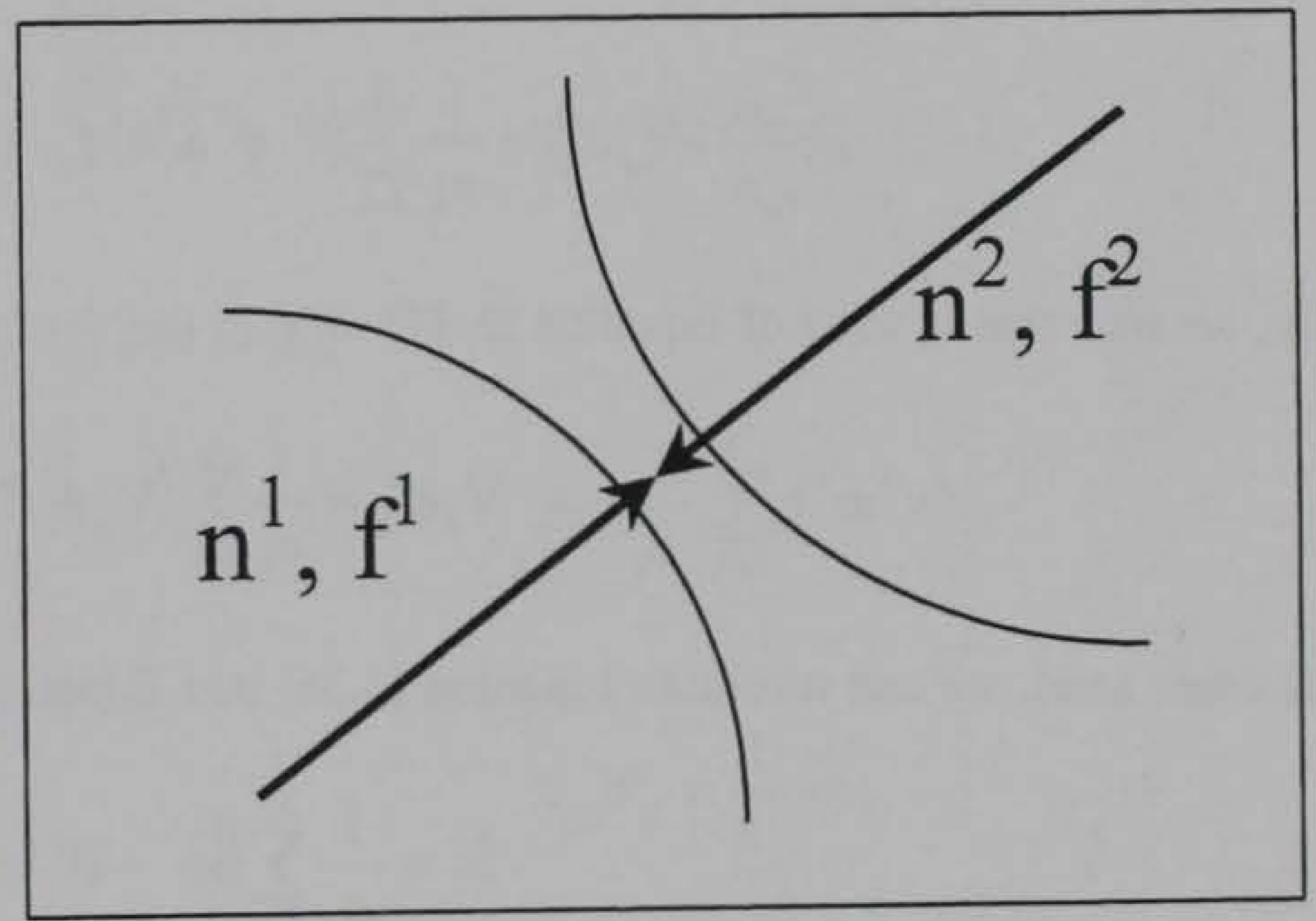

Figure 3.4: Labeling of Particles and their Contacts 
where $R$ is the radius of the particle and $V$ is the volume of the particle. It will be shown that a meaningful quantity is the volume-averaged particle stress:

$$
\bar{\sigma}_{i j}=\frac{1}{n_{s}} \sum_{k=1}^{K_{s}} \phi^{k} R^{k} \sum_{c=1}^{c} n_{i}^{c} f_{j}^{c}
$$

where

$$
n_{s}=\sum_{k=1}^{K_{s}} \phi_{k} V_{k}
$$

The averaged stress can also be computed from the multi-particle contact quantities as

$$
\bar{\sigma}_{i j}=\frac{1}{n_{s}} \sum_{l=1}^{L_{s}}\left(\phi^{1} R^{1} n_{i}^{1} f_{j}^{1}+\phi^{2} R^{2} n_{i}^{2} f_{j}^{2}\right)
$$

Because $\phi^{k}$ is the only function of position, the gradient of the averaged stress is given by (for uniform $n_{s}$ )

$$
\nabla_{j} \bar{\sigma}_{i j}=\frac{1}{n_{s}} \sum_{l=1}^{L_{s}}\left(\nabla_{j} \phi^{1} R^{1} n_{i}^{1} f_{j}^{1}+\nabla_{j} \phi^{2} R^{2} n_{i}^{2} f_{j}^{2}\right)
$$

Finally, we note that in view of Equation (3.37)

$$
\nabla_{j} \bar{\sigma}_{i j}=\frac{1}{n_{s}} \sum_{l=1}^{L_{s}}\left(\nabla_{j} \phi^{1} R^{1}+\nabla_{j} \phi^{2} R^{2}\right) n_{i} f_{i} .
$$

On the other hand, we can substitute Equation (3.36) into Equation (3.34) to get

$$
\dot{\bar{p}}_{i}=\frac{1}{\rho_{s}} \sum_{l=1}^{L_{s}}\left(\left(\phi^{1}-\phi^{2}\right) f_{i}^{1}\right)^{1} \text {. }
$$

The value of $\phi^{1}$ can be approximated as

$$
\phi^{\prime} \approx \phi^{1}+R^{1} n_{j}^{1} \nabla_{j} \phi^{1}
$$

or as 


$$
\phi^{\prime} \approx \phi^{2}+R^{2} n_{j}^{2} \nabla_{j} \phi^{2} .
$$

Subtracting these approximations we get:

$$
\phi^{1}-\phi^{2} \approx-R^{1} \nabla_{j} \phi^{1} n_{j}^{1}+R^{2} \nabla_{j} \phi^{2} n_{j}^{2} .
$$

In view of Equation (3.35),

$$
\phi^{1}-\phi^{2} \approx\left(R^{1} \nabla_{j} \phi^{1}+R^{2} \nabla_{j} \phi^{2}\right) n_{j}^{2}
$$

which gives, by substitution into Equation (3.45)

$$
\dot{\bar{p}}=\frac{1}{\rho_{s}} \sum_{l=1}^{L_{s}}\left(R^{1} \nabla_{j} \phi^{1}+R^{2} \nabla_{j} \phi^{2}\right) n_{j}^{2} f_{i}^{1} .
$$

By comparison of Equations (3.43) and (3.50) we have:

$$
\rho_{s} \dot{\bar{p}}_{i}=n_{s} \nabla_{j} \bar{\sigma}_{i j}
$$

or

$$
\nabla_{j} \bar{\sigma}_{i j}=\frac{\rho_{s}}{n_{s}} \dot{\bar{p}}_{i}
$$

From Equations (3.26) and (3.27)

$$
\dot{\bar{p}}=\frac{1}{\rho_{s}} \sum_{k=1}^{K_{s}} \phi^{k} m^{k} v_{i}^{k}
$$

Thus,

$$
\nabla_{j} \bar{\sigma}_{i j}=\frac{1}{n_{s}} \overline{m v_{i}}
$$

The right hand side of Equation (3.54) is dimensionally equivalent to the product of a density (mass per unit volume) and acceleration $\left(\dot{\vec{v}}_{i}\right)$. In fact, taking $m$ and $v_{i}$ to be independent quantities we have

$$
\nabla_{j} \bar{\sigma}_{i j}=\dot{\rho}_{i}
$$


where

$$
\bar{\rho}=\frac{\bar{m}}{n_{s}} .
$$

Finally, from Equation (3.55)

$$
\nabla_{j} \bar{\sigma}_{i j}=\bar{f}_{i}
$$

where, $f_{i}$ is the volume averaged force given by

$$
\bar{f}_{i}=\frac{1}{\rho_{s}} \sum_{k=1}^{K_{s}} \phi^{k} f_{i}^{k}
$$

Thus, the particle system when smoothed follows the same momentum balance as the continuum. 


\section{CHAPTER IV}

\section{Large Deformation Analysis using DEM}

\subsection{Introduction}

A major hindrance to developing a theory for large deformations in particulate media is that force and kinematics relationships are impossible to measure at the particle level in threedimensional granular flow. An alternative to physical measurements is to use a simulated particulate system from which quantities of interest can be computed. However, any continuum theory resulting from such simulated "measurements" would apply to the simulated media rather than to actual soil. This obvious point is important in view of the fact that the particulate simulations grossly simplify the real interactions. However, we cannot make experimental measurements at the particle level, interactions are based on inference from bulk behavior rather than direct experimental observation. Therefore, experimental verification of the numerical simulation is critical.

\subsection{Previous Work on DEM Validation}

There have been a limited number of reported attempts to validate DEM simulations with physical experiments. Rowell [43] used experimental data from Chapuis [9] to evaluate two-dimensional ellipse-based DEM simulations of dense and loose biaxial tests. Chapuis experiments consisted of assemblies of 400 to 600 cylinders, of $10-\mathrm{mm}$ length. Four particle diameters ranging from 19.05 to $38.76 \mathrm{~mm}$ were used. Rowell was able to successfully model the cylinder's behavior in the dense condition. However, he was unable to model the loose condition. Sakaguchi and Ozaki [44] compared DEM simulations with experiments of 856 cylinders of $10 \mathrm{~mm}$ diameter to evaluate the formation of arches (plugging) during the flow of granular material. Their DEM simulations yielded flow patterns that were in good agreement with experimental measurements. Rong, Negi, and Jofriet [42] performed numerical simulations and physical experiments of the flow behavior of bulk solids in bins. The particulate material used in the experiments consisted of hollow acrylic cylinders, $25.4 \mathrm{~mm}$ outside diameter. Experiments were conducted in sample sizes of either 195 or 780 cylinders. The numerically generated particle trajectories and velocities agreed well with the observed experimental flow behavior. The verification experiments to date have typically been performed on a relatively small number (less than 10,000 particles) of idealized shaped particles whose motions have been restricted to two-dimensions and whose size is much larger than that of real soils. The boundary effects in such cases are large and the details at the particle-level become critical. 


\subsection{Effects of Representation Grain Size Distribution}

Much work has been done to quantify the effects of particle stiffness, rotation, and shape on the DEM simulation [49], and [50]. Theoretical relationships have been developed to assign the particle contact properties such as spring stiffness and damping to reproduce bulk properties of the material being modeled [45], and [47]. However, very little work has been done to quantify the effects of using multiple particle sizes. More important is the question, for accurate DEM simulations, of how many particle sizes of a real soil's grain size distribution curve must be used to properly simulate the behavior of the soil. Granular soils consist of mineral fragments of various sizes and shapes. The ratio of largest to smallest particle diameter can be quite large. The representation of the grain size distribution curve can have a significant effect on the contact statistics and the mechanical behavior of both the actual soil and the simulation. To examine the effects of the representation of the grain size distribution curve on DEM results, a numerical study was conducted that consisted of three-dimensional simulations of sedimentation involving up to 93,000 particles having ratios of smallest to largest diameter ranging from 1:1 to 9.2:1. An actual grain size distribution curve of Treasure Island sand (Figure 4.1) was used as the basis for the simulations. The simulations consisted of dropping the computer particles into a box and allowing the particulate mass to settle due to is own weight. The focus of the investigation was the distribution of porosity in the simulated media, particularly near rigid boundaries. Evaluation of the initial porosity states of the particle masses included variations in porosity, distribution of contacts, and tendency of the packing to crystallize near hard rigid boundaries.

The Treasure Island sand was modeled using four simulations having $1,2,5$, and 10 particle sizes to represent the grain size distribution curve. The weight-based grain size distribution curve was converted to a discrete probability distribution function represented by $M$ different sized particles. The probability of a certain grain size, $P\left(D_{x}\right)$, occurring in a sample given is given by [26]

$$
P\left(D_{x}\right)=\frac{1}{D_{x}^{3} \sum_{k=1}^{M} \frac{1}{\frac{D_{00(2 k-1)}^{3}}{M}}}
$$

where $D_{x}$ is the grain size diameter for a particular location on the grain size distribution curve (e.g. $\left.D_{50}\right)$. This equation assumes that the mass of the soil is distributed equally among the $\mathbf{M}$ different particle sizes. Clearly, as $\mathrm{M}$ increases, the simulation more adequately represents the actual soil.

For this study, a constant sample mass of one gram was used. The number of particles required to represent a grain size distribution increases as more particle sizes are used, as 
shown in Table 4.1. The increase in particles with $\mathrm{m}$ is due to the fact that more of the smaller size grains are being modeled as more particle sizes are used. Figure 4.2 plots the probability of particle occurrence versus grain size for each simulation of the grain size distribution. For well-graded soils, many more particles are needed to statistically simulate the particle distribution than required by uniform soils. Thus, the applicability of particle methods to model real soil media becomes greatly limited as the grain size distribution becomes broader.

The representation of the grain size distribution curve can have a significant effect on the contact statistics and the mechanical behavior of the simulation. This fact is illustrated in Figure 4.3, which shows the particle packing of a simulation of only one-grain size as opposed to a simulation that used five different grain sizes. The simulation using only a single size particle size produces a very uniformly structured packing, while the simulation using five particle sizes produces a packing that is similar to real granular soils.

Figure 4.4 shows how various representations of the grain size distribution curve effect the variation of planar porosity from a rigid boundary. A key finding is that the representation of the grain size distribution has a significant effect on the porosity distribution near boundaries. The more particle sizes used to represent a particular distribution, the less pronounced the boundary effect would be. When the soil is represented by only one or two grain sizes, the tendency is for the particles to align with the rigid wall. This alignment propagates inward from the wall for several particle diameters. As the number of particle sizes is increased, the alignment becomes limited to a distance of approximately one particle diameter from the wall.

The average coordination numbers (the average number of contacts per particle) for each particle size in the four simulations is shown in Figure 4.5. The coordination number is defined as the number of contacts a particle makes with its surrounding particles. For each individual simulation, the coordination number is proportional to the surface area of a particle. Another interesting point is that the coordination number increases as the number of grain sizes increases, especially for the larger particles. This would indicate that by modeling more of the fine material tighter packing could be achieved.

For the remaining simulations it was decided that five particle sizes would be used to represent the grain size distribution curve. This provided a realistic looking packing structure, while keeping the simulations to a reasonable number of particles.

\subsection{Soil Plowing Simulation}

A soil plowing experiment consisting of the horizontal translation of a vertical wall through a uniform Ottawa 20-30 sand was performed. The experiment was configured as a plane-strain test whereby motion of the wall was in one plane and the sand was confined between rigid glass plates. The DEM simulation was three-dimensional in that, particles were free to move out-of-plane to the extent permitted by the boundaries. Comparison between simulation and experiment focused on sand deformation, velocity of individual points within the mass, and the total horizontal force on the plow during advance. This particular problem was 
chosen for study because the expected large deformations, development of shear bands and slope instability are characteristic of a large class of large deformation problems.

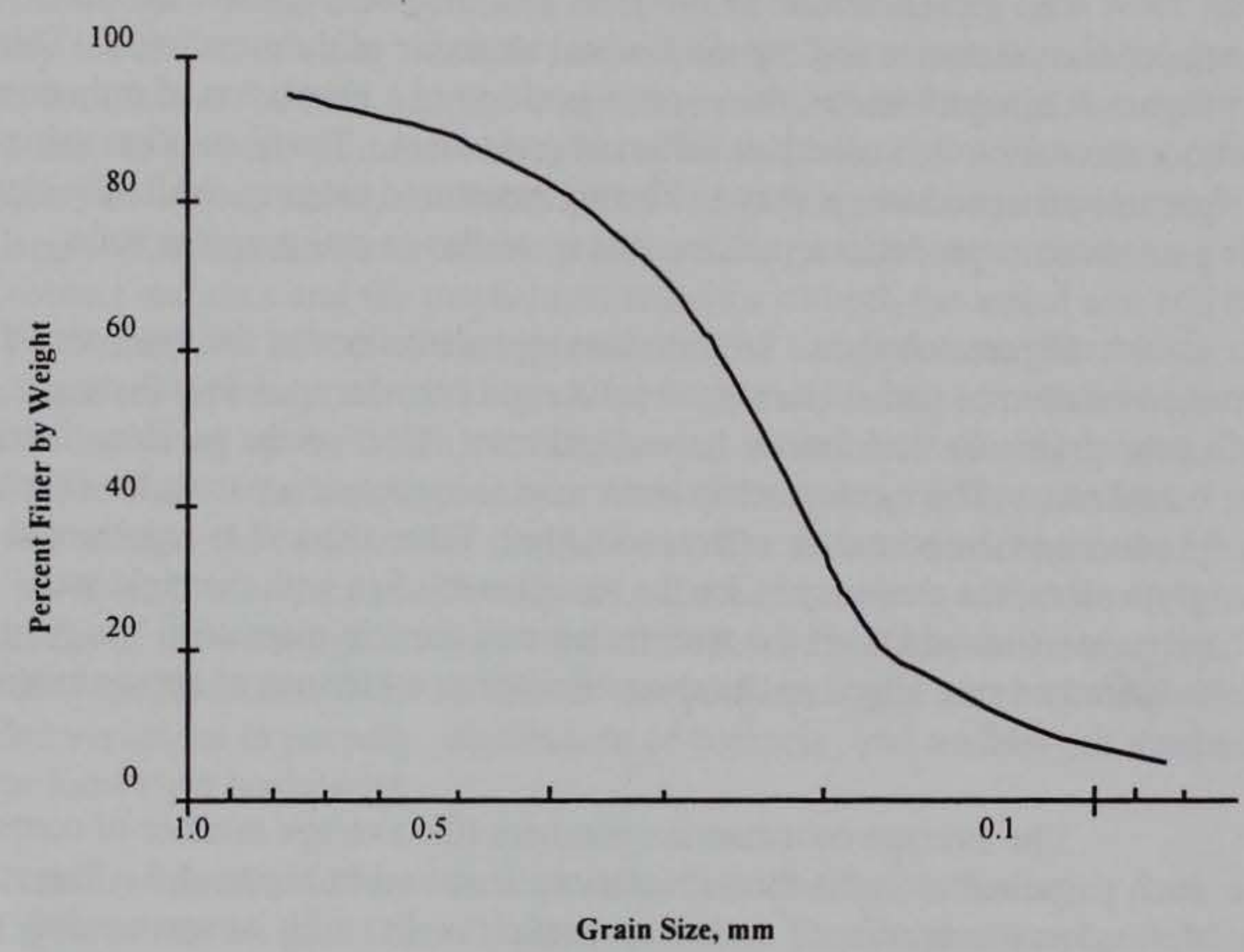

Figure 4.1: Treasure Island Grain Size Distribution Curve

\subsection{Plowing Experiment}

The physical model consisted of a rectangular vessel (300-mm in length $\times 150 \mathrm{~mm}$ in height $\times 8.5 \mathrm{~mm}$ in width) with transparent glass sides. However, only a portion of the vessel was used, providing test dimensions as shown in Figure 4.6. The plow was attached to the underside of a small, four-wheeled trolley, which traveled along two rails. The sand was placed in the vessel using a deep-throated funnel. After the sand was placed to the desired height, the left end wall of the vessel was removed, thereby allowing the sand to flow out and form a slope at the soil's angle of repose. As the plow advances toward this wall, sand can run out of the vessel. Thus, a nearly constant slope angle is maintained. The trolley is displaced at a constant rate of $2.5 \mathrm{~cm} / \mathrm{sec}$. 
Video images of the plowing experiments were recorded in real time and analyzed using a computer vision system. An image processing and analysis program called "Particle Tracer" [41] semi-automatically obtained the shape of the sand surface and location of the plow with time, and the displacement trajectories of selected sand particles during plow advance.

To characterize the displacement field, individual sand particles are coated with a fluorescent dye. The experiment is recorded on video under UV light. The bright fluorescent tracer particles can be segmented from the other particles in the digitized images using a simple thresholding operation. All pixels with a grayscale value greater than some threshold value are marked as foreground regions and their grayscale pixel value is set equal to 255 (white). All other pixels are marked as black (grayscale value $=0$ ). The plow was coated with the same fluorescent dye as the tracer particles and a white background was used behind the vessel. This enables Tracer to also determine the soil surface profile and the location of the plow in each image.

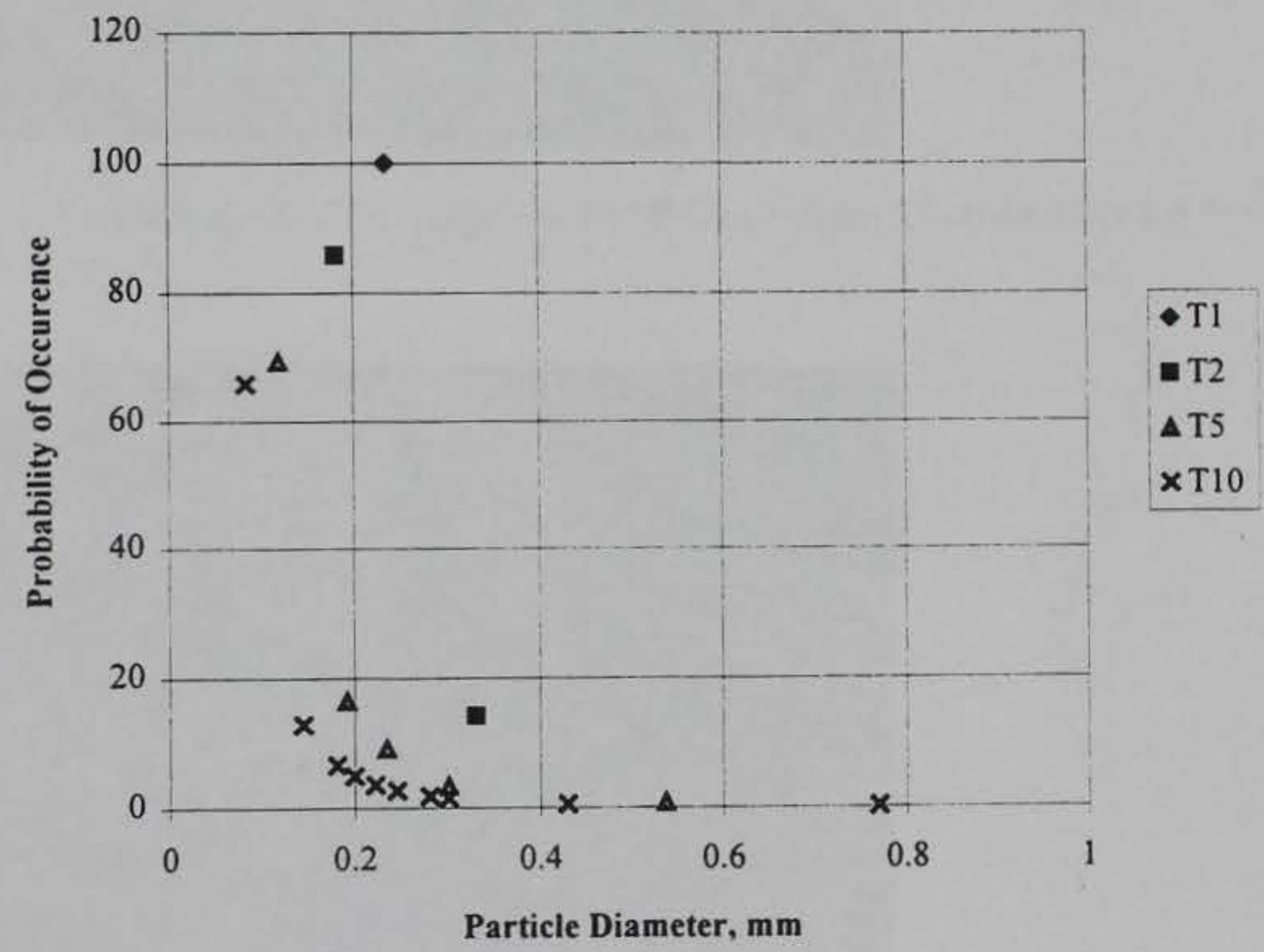

Figure 4.2: Probability of Particle Size Occurrence

Table 4.1: Number of Particles

Number of Particle Sizes

(M)

1

2

5

10
Number of Particles in Sample

28,139

35,156

62,526

93,174 

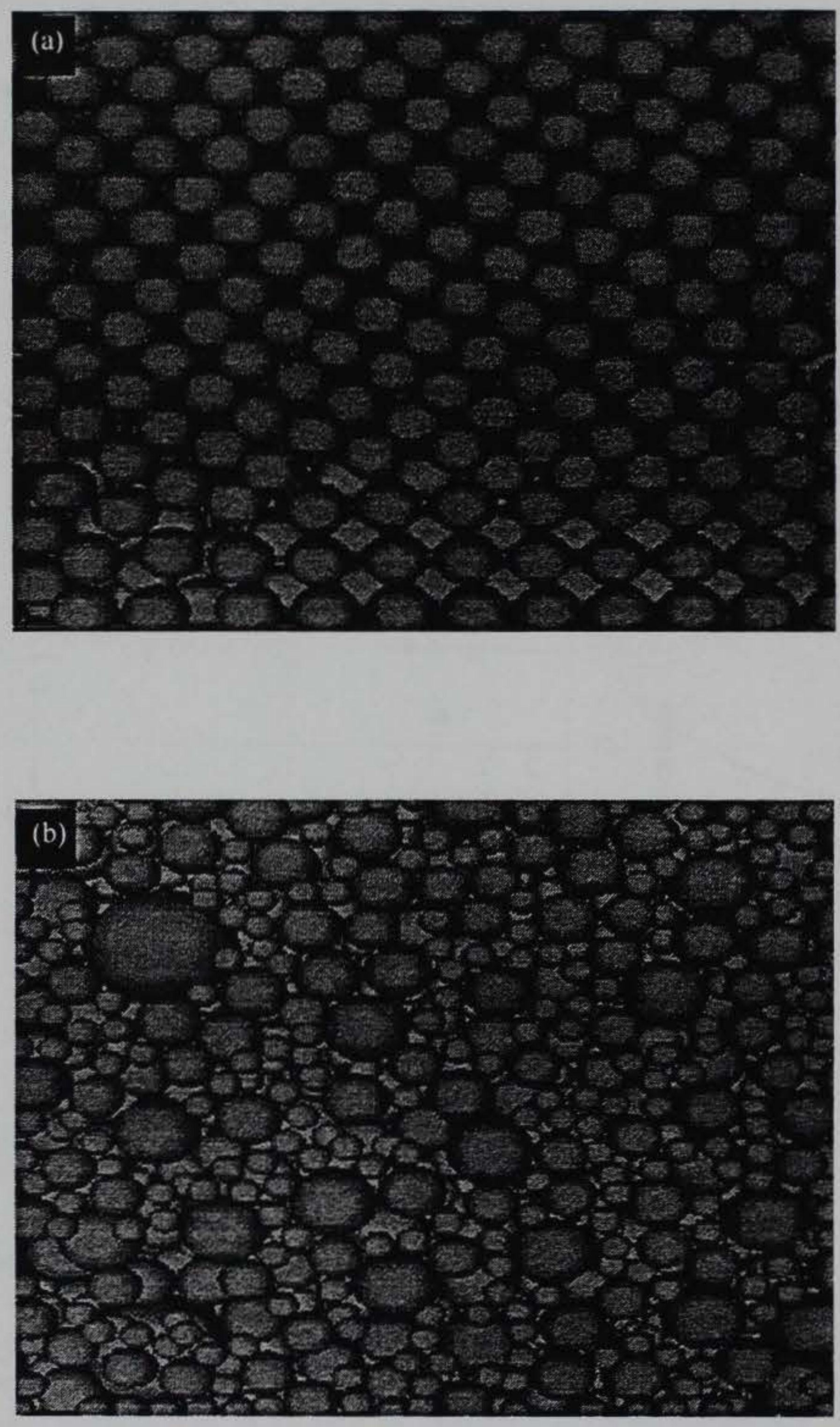

Figure 4.3: Particle Packing using (a) One Grain Size (b) Five Grain Sizes 


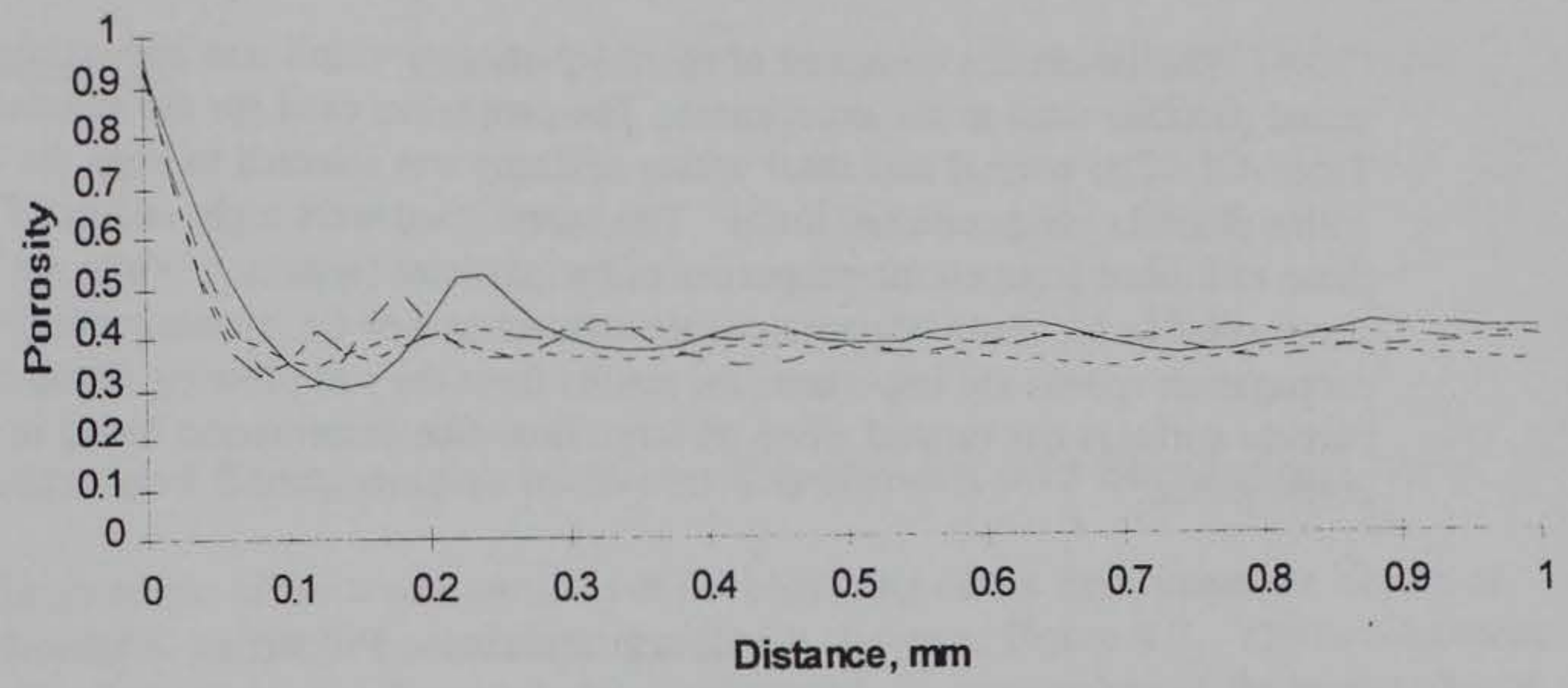

T1

T2 T5 T10

Figure 4.4: Effects of Representation of Grain Size Distribution on Porosity

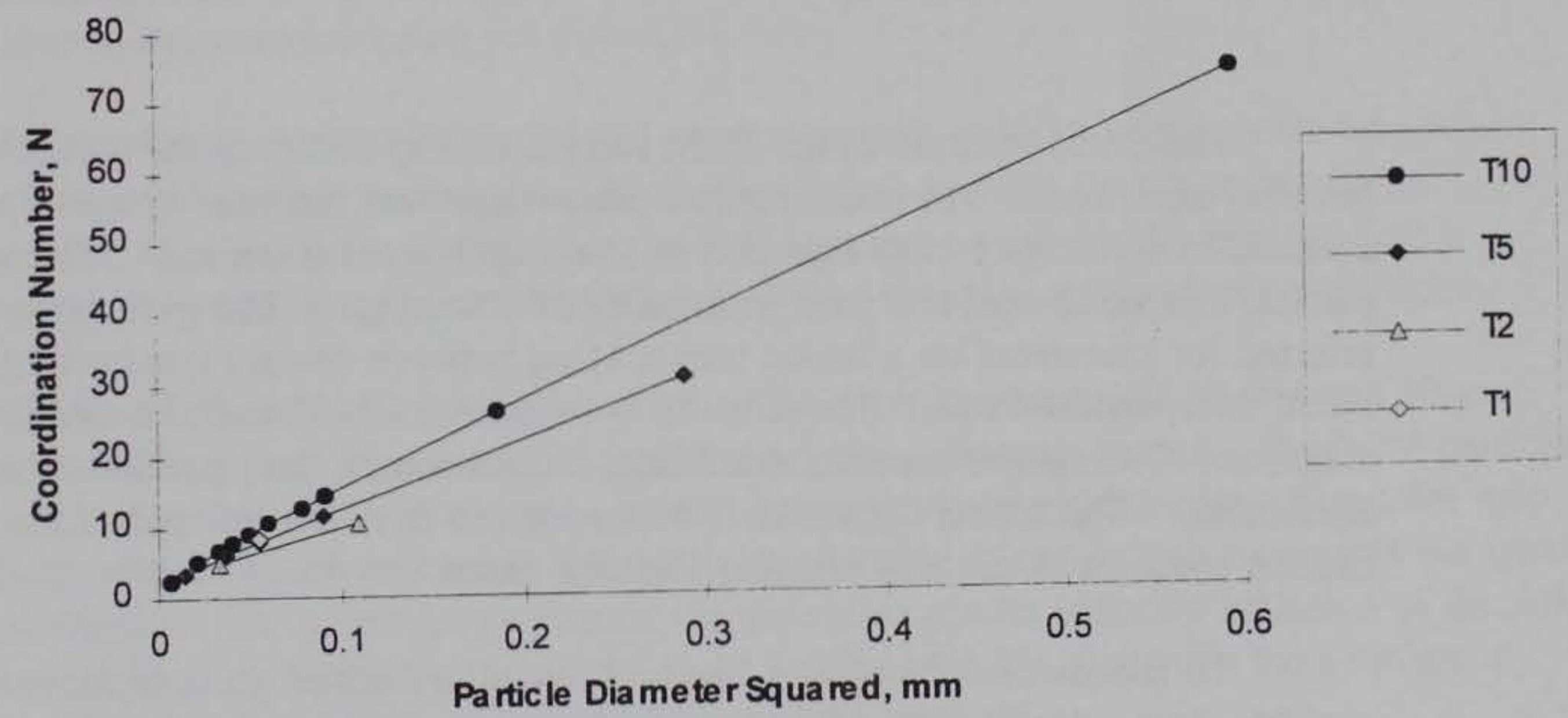

Figure 4.5: Effects of Representation of Grain Size Distribution on Coordination Number 


\subsection{Simulation Description}

The simulation consisted of 66,544 particles, which was approximately the number of actual particles used in the experiment. The properties used for the simulation are shown in Table 4.2. The normal and shear spring stiffness was selected to keep the critical time step within feasible computational limits. The values used were eight orders of magnitude less than those estimated from elastic properties of the particles (quartz). While the low particle stiffness is not suitable for study of particle-scale mechanisms or for dynamic computations where wave propagation speeds are important, the results from the soil plowing simulation indicated that particle stiffness has limited effect on large flow-like deformation found in the problems under investigation.

Table 4.2: Simulation Properties

$\begin{array}{ll}\text { Particle Shape } & \text { spherical } \\ \text { Specific Gravity } & 2.65 \\ \text { Contact Stiffness: } & \\ \text { Normal } & 1.4 \mathrm{~kg} / \mathrm{m} \\ \text { Shear } & 0.4 \mathrm{~kg} / \mathrm{m} \\ \text { Contact Friction Angle } & 15 \mathrm{degrees} \\ \text { Particle to Wall Friction } & 20 \text { degrees } \\ \text { Coefficient of Restitution } & 0.04 \\ \text { Plow Advance } & 2.5 \mathrm{~cm} / \mathrm{sec} \\ \text { Time Step } & 2 . \mathrm{E}-5 \mathrm{sec}\end{array}$

The initial creation of the DEM sample closely followed the procedures used in the physical experiment. An initial particle assemblage was obtained by randomly creating particles in accordance with Equation (4.1) using five particle sizes. Table 4.3 shows the particle diameters used and their probability of occurrence. The particles were then randomly selected for placement on a lattice with spacing between centers large enough to minimize initial Interparticle forces. The particles were then "rained" into the simulated rigid-wall container. The sample creation simulation continues until the particles achieve static equilibrium. The lateral constraint is then removed from the left end of the simulated test box causing particles to run out, creating a natural slope.

To reduce the size of data files and to depict discrete particle data as continuum field variables (e.g., density, velocity and velocity gradients, and stresses), data were mapped to a grid as weighted averages using the smoothing technique described in the Chapter 3 . After smoothed averages are computed for each grid location; data visualization is accomplished using standard finite element post processing software. 
Table 4.3:Discrete Ottawa 20-30 Grain Size Distribution

Percent Passing:

10

30

50

70

90
Particle Diameter

$.63 \mathrm{~mm}$

$.66 \mathrm{~mm}$

$.70 \mathrm{~mm}$

$.72 \mathrm{~mm}$

$.75 \mathrm{~mm}$
Probability of Occurrence

25.90

22.52

18.88

17.35

15.35

\subsection{Results and Comparisons between Experiment and Simulation}

The locations of the tracer particles at the beginning of the experiment, at $10 \mathrm{~mm}$ of plow displacement, and at $20 \mathrm{~mm}$ of displacement are shown in Figure 4.7. The development of a soil mound ahead of the advancing plow is observed. A comparison of the trajectories of tracer particles numbered 1,2, 20 and 21 (as identified in Figure 4.7) to the closest particle in the DEM simulation for the first $20 \mathrm{~mm}$ of plowing is shown in Figure 4.8.

The simulated particles and tracer particles follow a similar trajectory. However, the simulated particles have a smaller net displacement than the real soil particles, particularly in the vertical direction. This is in part due to the softness of the simulated particles. If the particles had greater stiffness, they would tend to move over each other rather than compress between each other. Analysis of contact information at $10 \mathrm{~mm}$ of plow movement indicated that $46 \%$ of the contacts of the total simulation had penetrations greater than $1 \%$ of the particle diameter and $0.8 \%$ of contacts had penetrations greater than $5 \%$ of the particle diameter. The highest penetrations occurred near the plow face.

Figure 4.9 shows the horizontal and vertical particle velocity components for several simulated-observed particle pairs located in the plow zone over the first $20-\mathrm{mm}$ of plow displacement. This plot demonstrates that the DEM particles are typically moving with a velocity magnitude that is approximately $5 \mathrm{~mm} / \mathrm{s}$ slower than the observed tracer particles.

Figures 4.10 and 4.11 reveal the shape of the plowed material at $10 \mathrm{~mm}$ and $20-\mathrm{mm}$ plow displacement. Since the individual particles are too numerous to show, the DEM particle data was smoothed. A color spectrum is used to represent the mass densities. The color map ranges from yellow, which represents relatively low density, to red, which represents the areas of highest density. The green fringe around the perimeter results from the inclusion of the void space outside the slope in the averaging. Figures $4.10 \mathrm{a}$ and $4.11 \mathrm{a}$ show that the simulation indicates a zone of high densification just in front of the plow tip. 


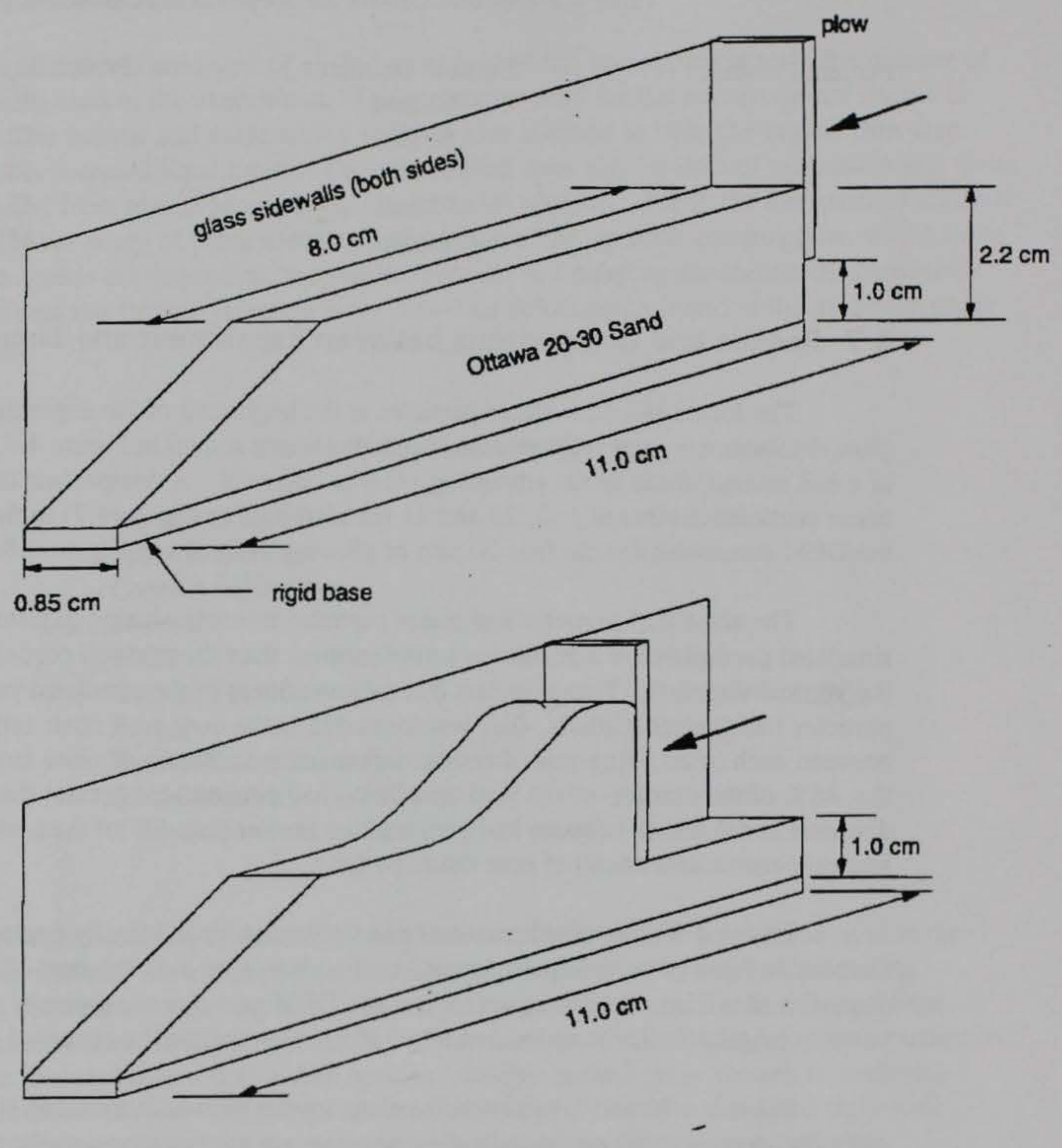

Figure 4.6: The Plowing Problem 
The contour lines shown on the interior of the slope in Figures 4.10 and 4.11 are contours of particle velocity magnitude in $\mathrm{mm} / \mathrm{s}$. A comparison of the experiment and simulation results reveals that the complex deformation patterns observed in the experiments in the vicinity of the plow are captured well by the simulation. As the plow moves into the soil, a zone of intense shearing forms at the tip of the plow and propagates diagonally upward to form a shear band. This zone of shearing moves laterally with the plow, forming a moving velocity discontinuity. The location of the shear band is identified by the closely spaced velocity contours. In the region to the left and below the $10 \mathrm{~mm} / \mathrm{s}$ contour, the particles are essentially stationary. In the region to the right and above the $23.5 \mathrm{~mm} / \mathrm{s}$ contour, sand is moving as a deforming plug ahead of the advancing plow. The location of the experimental contours is virtually identical to those obtained from the DEM simulations, indicating that the DEM model has predicted the location, width, and the motion of particles in the advancing velocity discontinuity well.

Plow force comparisons were made between experiment of the Ottawa 20-30 sand and the DEM simulation. An additional plowing experiment using glass beads was also used to compare plow forces. The glass beads had particle sizes similar to the Ottawa 20-30 sand and the DEM simulation. Figure 4.12 shows the resulting plowing forces from the simulation and the two experiments. The Ottawa 20-30 sand had the highest plowing force, while the glass bead experiment provided the lowest plowing force. The low plowing force obtained during the glass bead plowing experiment is attributed to the glass bead's great ability to roll compared to the Ottawa 20-30 sand. One interesting observation is that glass beads behave quite differently from the Ottawa 20-30 sand. Even though the Ottawa 20-30 sand is considered well-rounded sand, it appears that it does not have the same particle rolling characteristics as the glass beads. The simulation plowing force was less than that of the Ottawa 20-30 sand. This was attributed to low stiffness of the particles in the simulation.

The DEM simulation particle data was smoothed to compute the spatial velocity gradient tensor to examine zones of shearing and rotation within the simulation. Figure 4.12 shows the $D_{12}$ term of the rate deformation tensor at 1 and $2 \mathrm{~cm}$ of plowing. The rate deformation tensor was computed from the spatial velocity gradient and is defined as:

$$
D_{i j}=\frac{1}{2}\left(L_{i j}+L_{j i}\right)
$$

This figure shows that a zone of high shear is occurring just in front of the plow tip. Figure 4.14 shows the $w_{12}$ term of the spin tensor. The spin tensor is also computed from the spatial velocity gradient and is defined as:

$$
w_{i j}=\frac{1}{2}\left(L_{i j}-L_{j i}\right)
$$


It is interesting to note that even though individual particle rotations were prevented, the particulate system still had macroscopic rotations.

A final observation should be made regarding the shape of the slope face. In the experiments the slope face was linear, while in the DEM simulation it was slightly concave downward. This can be attributed largely to the fact that particle rotation was suppressed in the simulation. This suggests that simulations involving surface slope failures such as avalanches or hopper flow should include particle rotation.

\subsection{Trap Door Simulations}

\subsubsection{Description of Experiment}

The trap door experiment consisted of filling a narrow box of dimensions $8.5 \mathrm{~mm}$ thick by $50.8 \mathrm{~mm}$ wide by $89.3 \mathrm{~mm}$ high as shown in Figure 4.15 with 15 grams of dry Ottawa 20 30 sand. An experiment was also performed using glass beads. A small sliding gate located at the bottom of the box was removed, creating a small rectangular opening at the center of the base. This particular problem was chosen for study because the large deformations, development of shear bands, and slope instability are created by gravity flow, which differs from the failure mechanisms in the plowing simulations. Additionally, the trap door simulation creates zones where high velocity gradients occur with a relatively low density of particles. The trap door simulation allows for the evaluation of the smoothed system ability to capture these sharp changes in the velocity field in sparse particle systems.

\subsubsection{Comparison of Results}

The simulation consisted of 33,270 particles, which represented 15 grams of Ottawa 20-30 sand. The soil and interface properties in the trap door simulations were the same as in the plowing simulations except that the particle and wall friction angles were varied to evaluate their impact on the results. All simulations were run to at least one second of real-time. The simulation in which the interparticle friction was 25 degrees and the particle-wall friction was 22 degrees, which had the fastest flow rate, was taken out until material had flowed through the box. During the Ottawa 20-30-sand experiment, flow ceased at 2.6 seconds, while in the glass beads experiment flow ceased at 4.3 seconds. Inspection of the tracer data revealed that the glass beads had more horizontal movements than the Ottawa 20-30 sand and the angle of repose was much flatter than that of the Ottawa 20-30 sand. In all cases the DEM simulation's flow rate was significantly slower than that of the actual Ottawa 20-30 sand or the glass beads. Flow in the DEM simulation ceased at 5.8 seconds. This was expected because by preventing particle rotations in the simulation the overall particulate structure was more stable and flow though the trap door was very difficult to maintain. Figure 4.16 shows the surface profile from the experiments and the DEM simulation at two seconds. Clearly the simulation did not flow at the rate of the Ottawa 20-30 sand or the glass beads. However, the pattern of flow for the simulation was similar to the Ottawa 20-30 sand. Figures 4.17 and 4.18 show the evolution of 
the $D_{12}$ of the rate deformation tensor. This shows horizontal movements towards the trap door along the top surface. Additionally, there is a zone of high horizontal acceleration just above the trap door opening.

\subsubsection{Conclusions}

A comparison was made between laboratory experiments involving very large discontinuous deformations in sand and numerical simulations using a large-scale DEM computation. The magnitude of the simulation provides a unique opportunity to assess the validity of the DEM based on experimental results. The simulation captures the behavior of a particulate "continuum" while the small-scale test permits a one-to-one correspondence between particle gradation in the simulation and the test. The agreement between the experimental and simulated particle motions in the plowing experiment indicates that many details not captured by the simplistic particle interaction model may not be relevant in statistically large assemblies.

These simulations represent the present limiting scale at which DEM can be used on a one-to-one basis. Even with improved computing hardware and algorithms, simulation capacity can be increased by only factors of 10 to 100 . Such increases still do not translate into appreciably larger physical experiments. Yet it has been demonstrated that a middle ground does exist between computationally feasible DEM simulations and small-scale experiments that are applicable to engineering scale problems. 

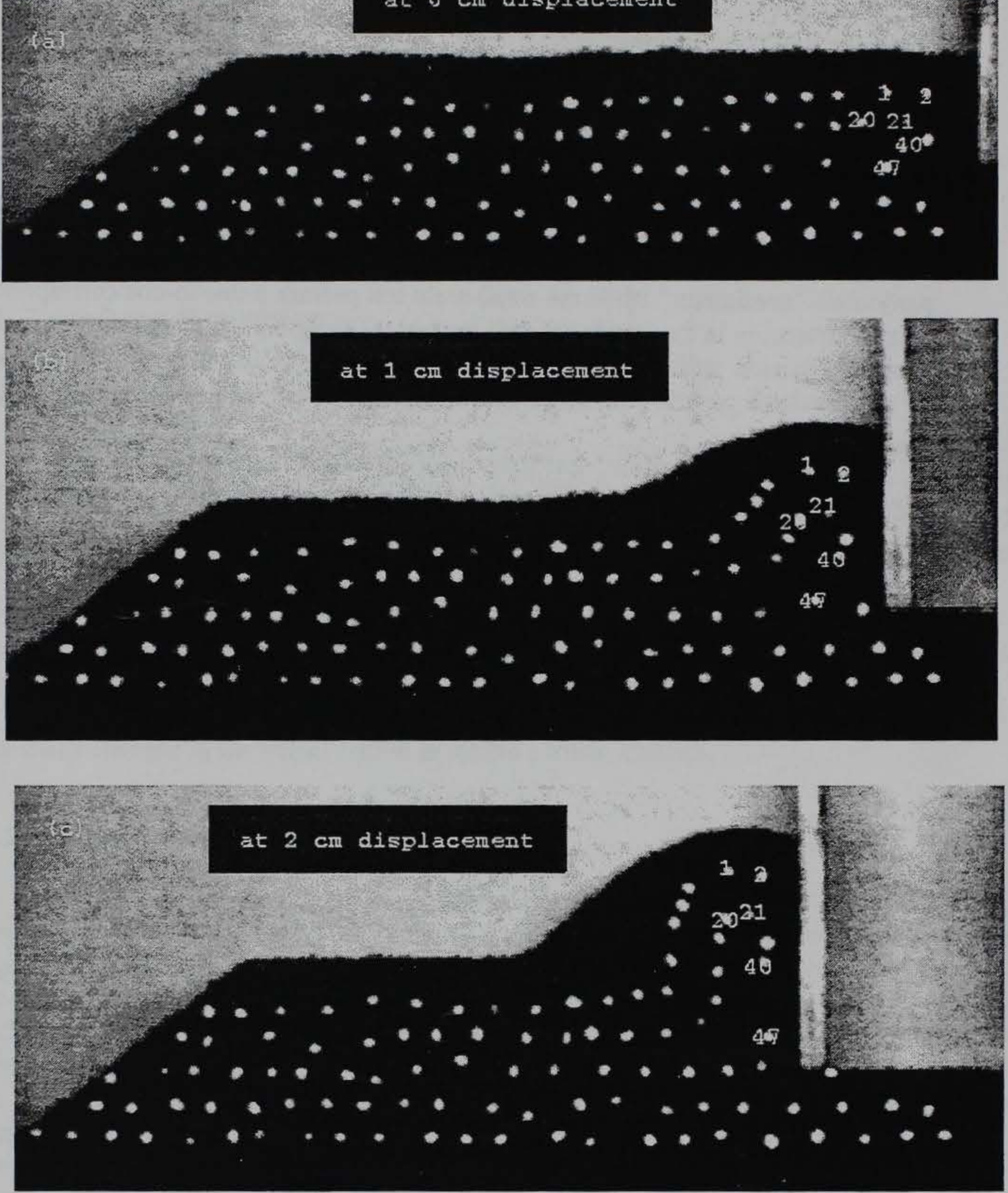

Figure 4.7: Plow Experiment at (a) $0 \mathrm{~cm}$ Displacement, (b) $1 \mathrm{~cm}$ Displacement, (c) $2 \mathrm{~cm}$ Displacement 


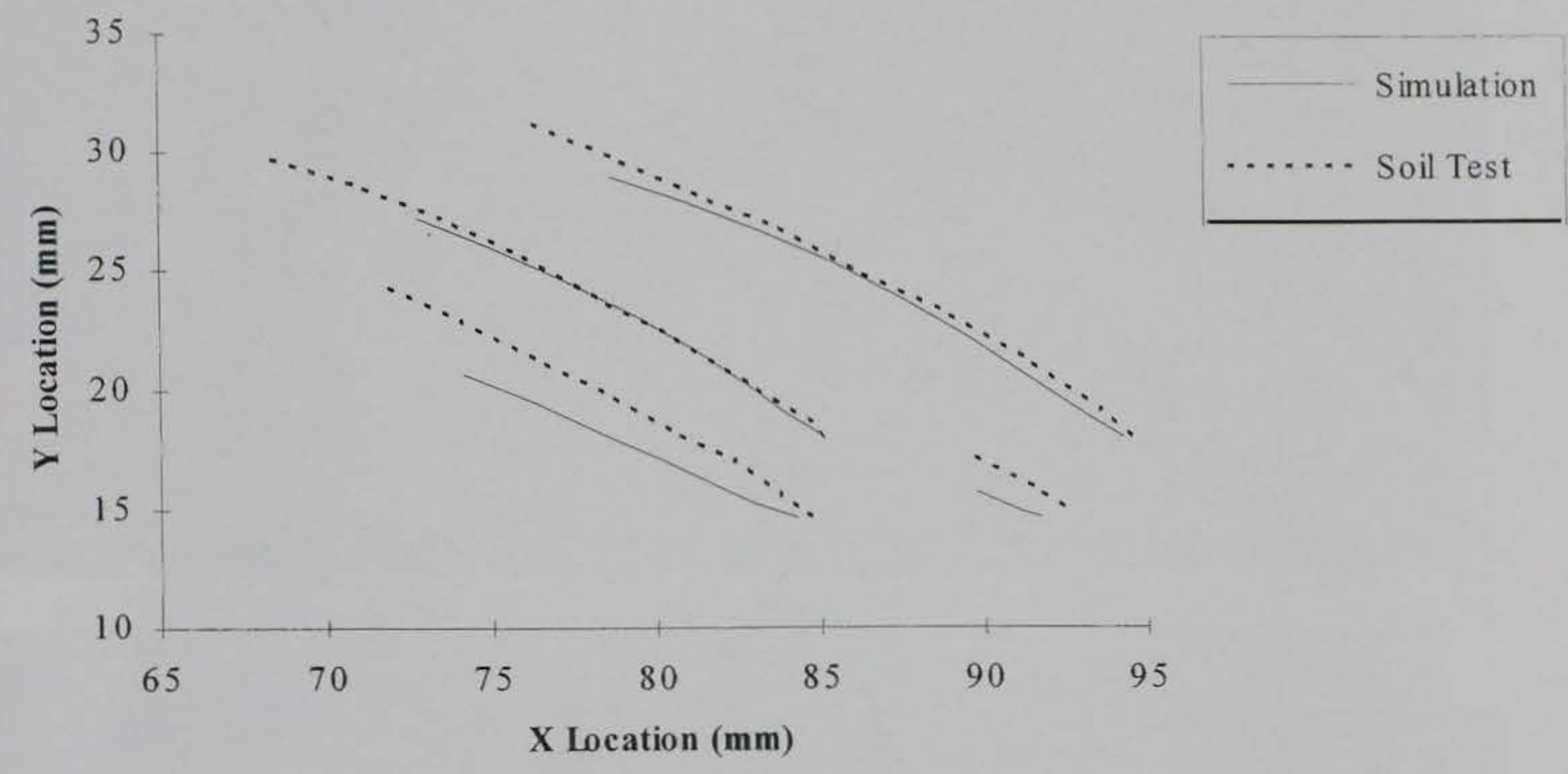

Figure 4.8: Comparison of Particle Traces over $2 \mathrm{~cm}$ of Plowing

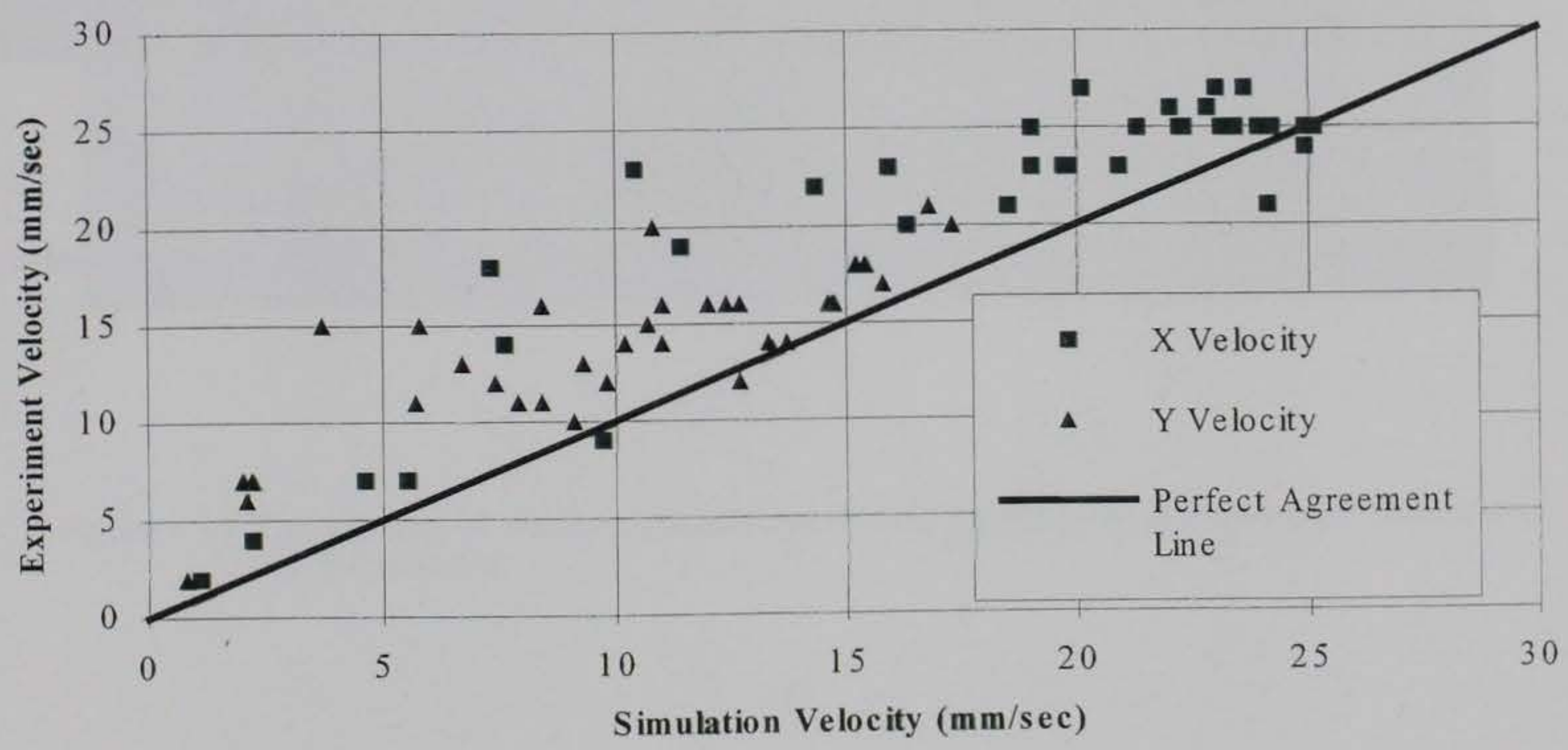

Figure 4.9: Experimental and Simulation Particle Velocities 

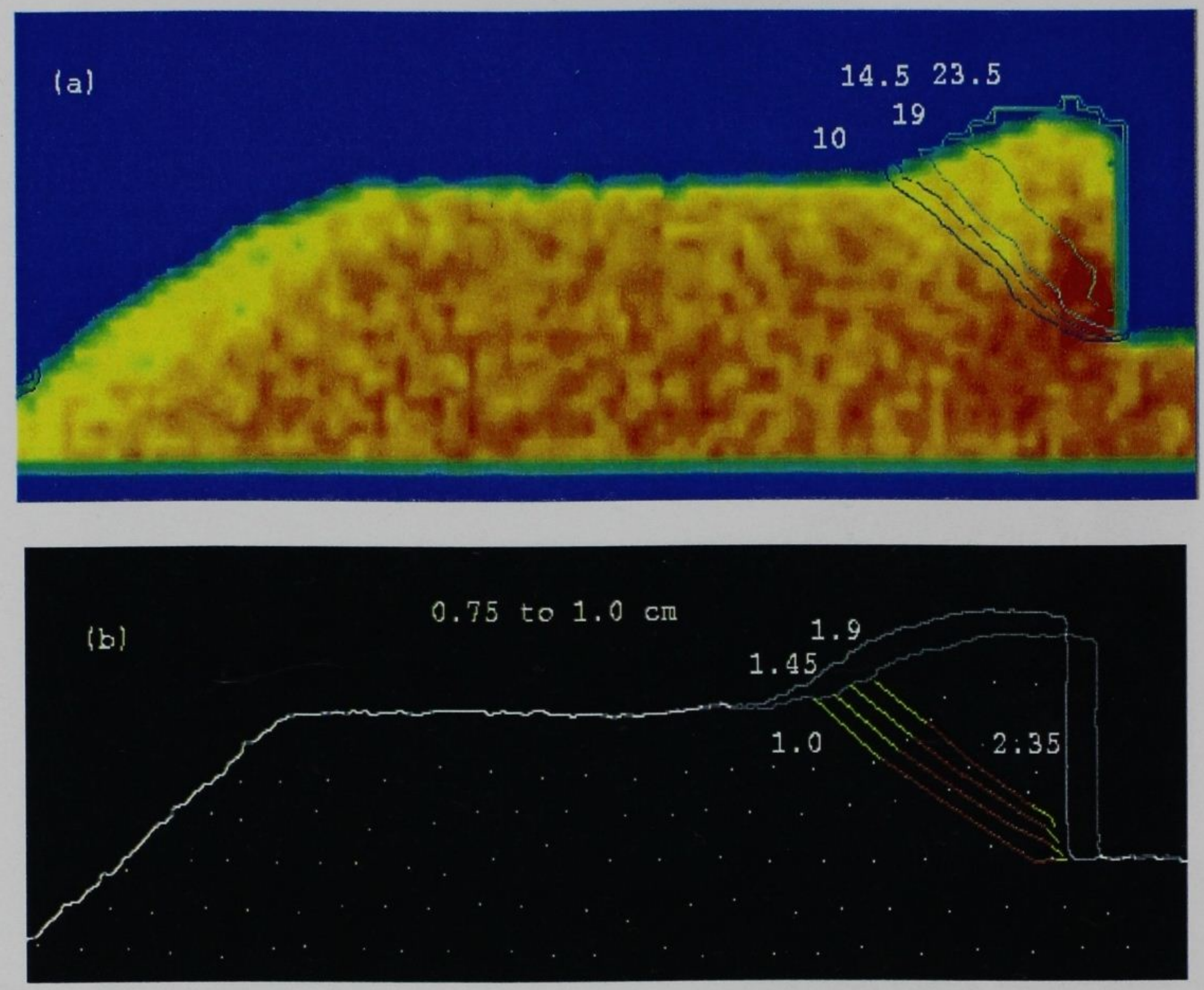

Figure 4.10: Comparison of Velocity Magnitudes Particle during $1 \mathrm{~cm}$ of Plowing (a) Simulation (b) Experiment 

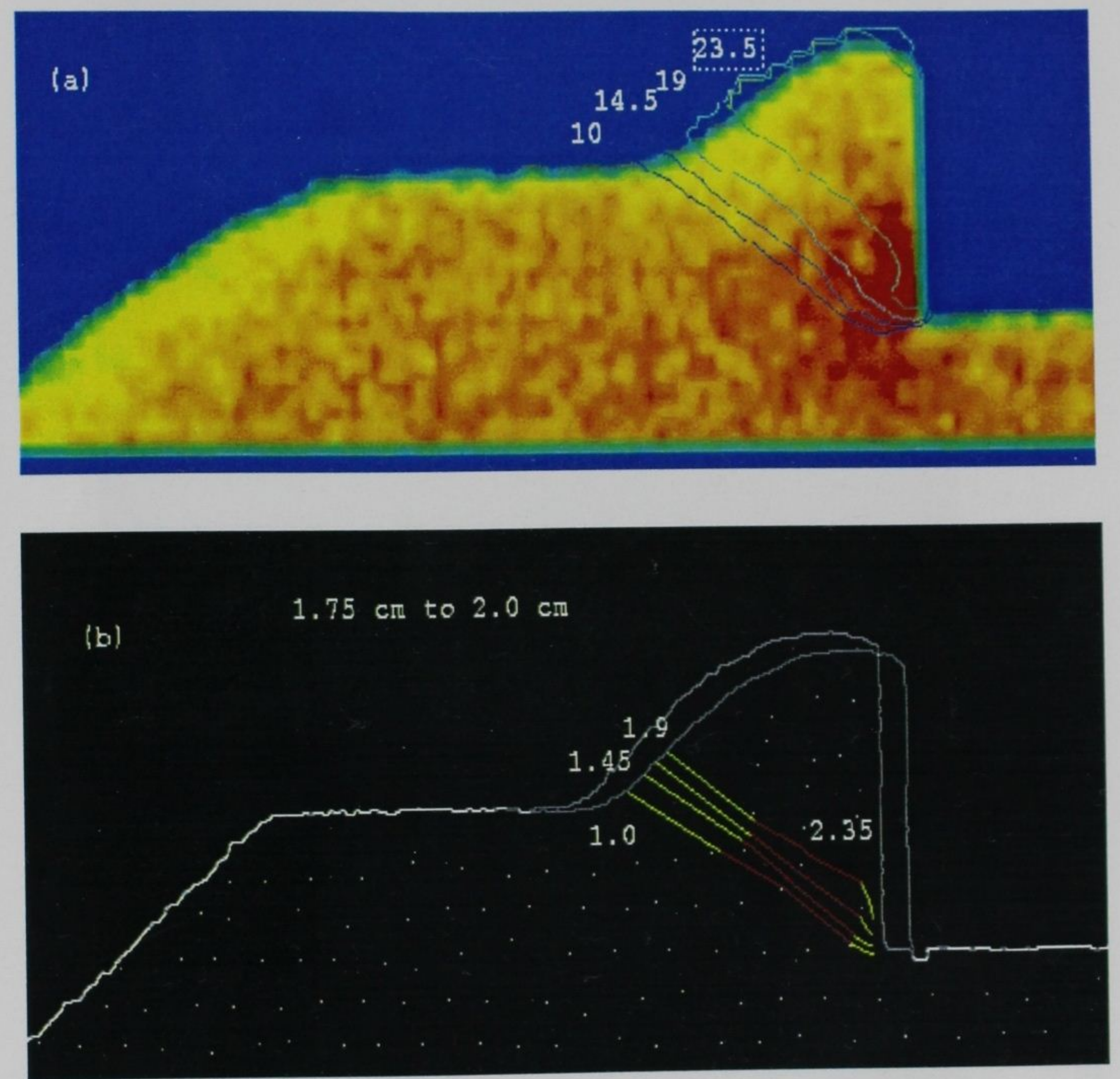

Figure 4.11: Comparison of Velocity Magnitudes Particle during $2 \mathrm{~cm}$ of Plowing (a) Simulation (b) Experiment 


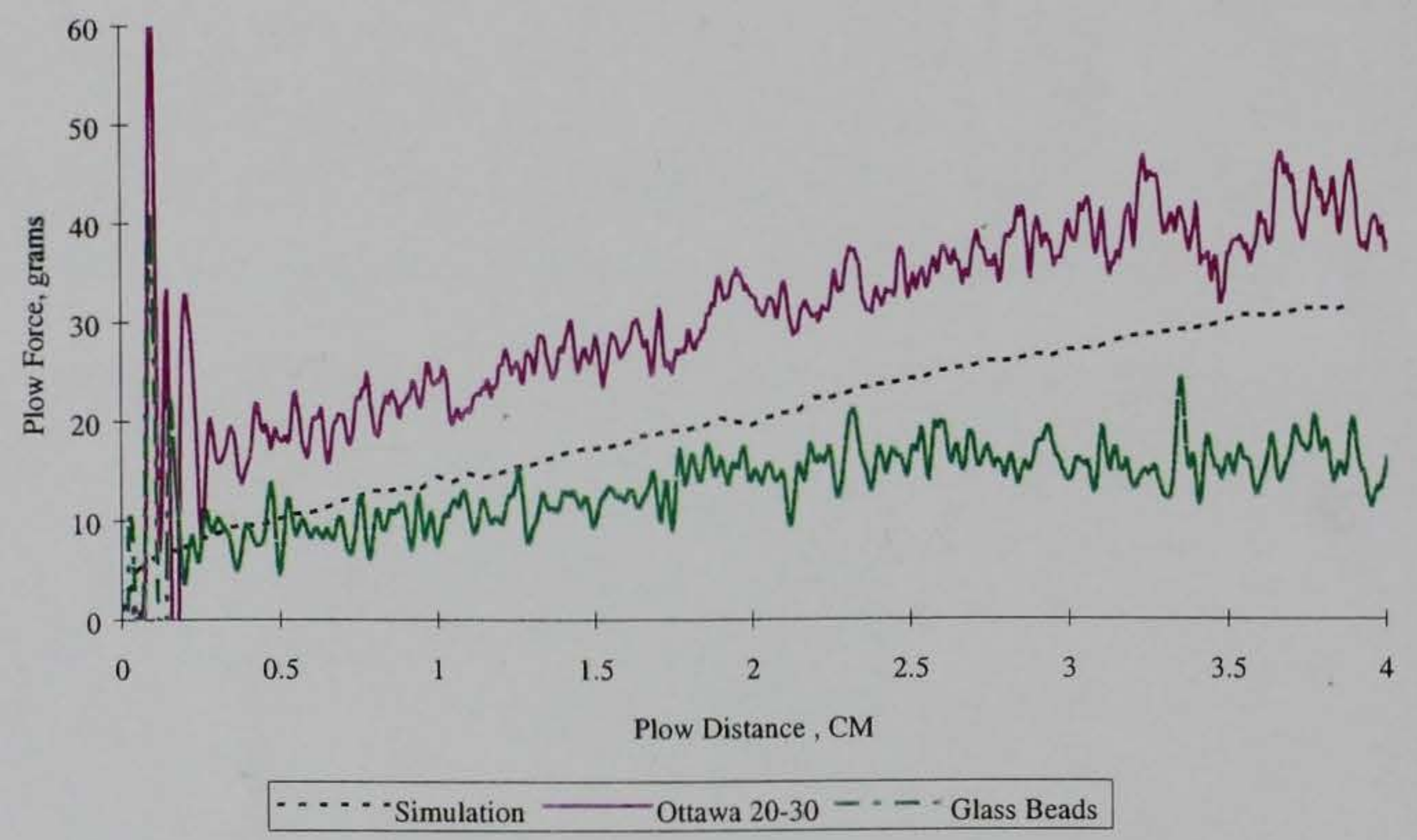

Figure 4.12: Plow Forces 

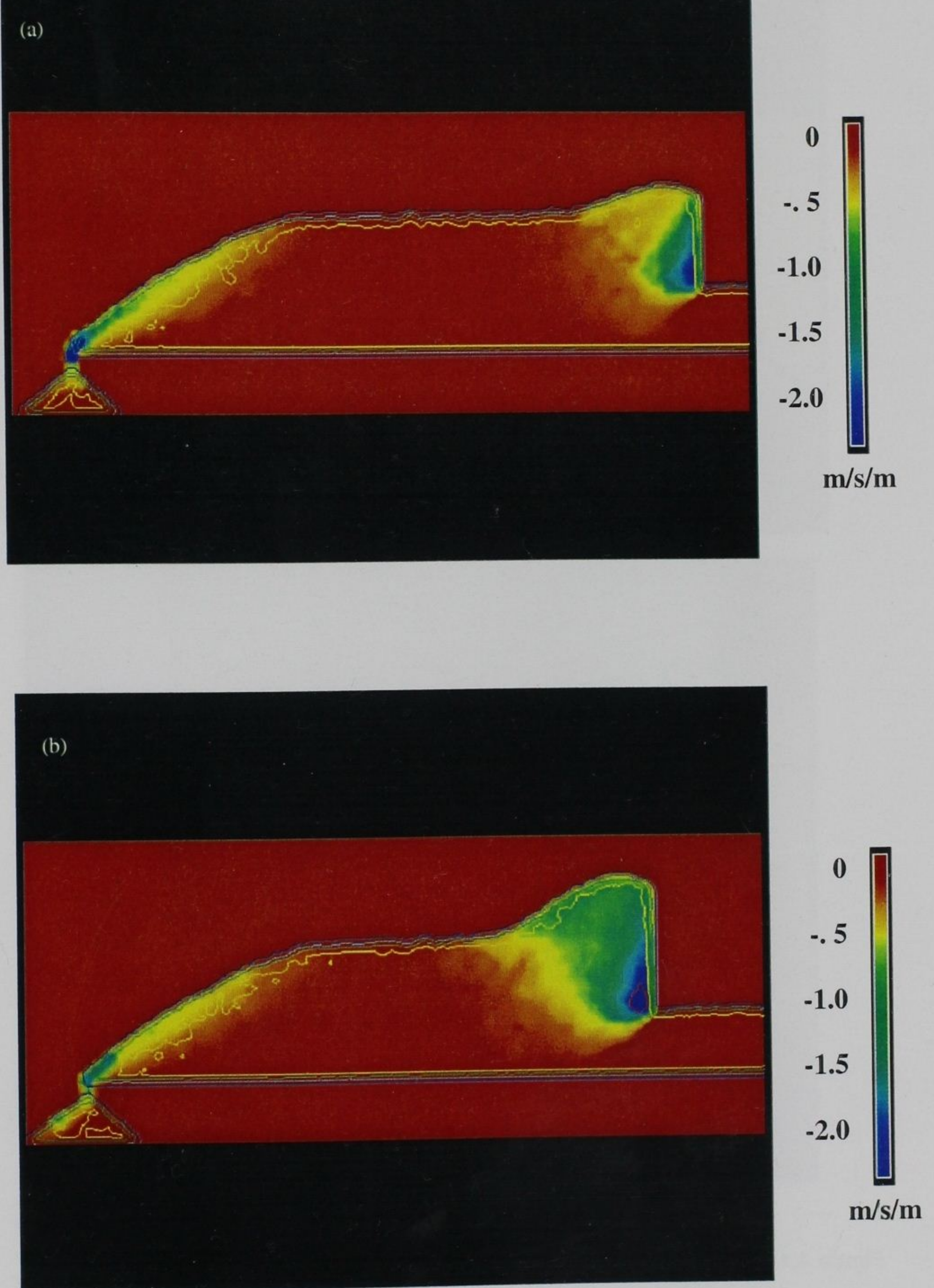

Figure 4.13: Shear Deformation Rate at (a) $1 \mathrm{~cm}$ of Plowing (b) $2 \mathrm{~cm}$ of Plowing 

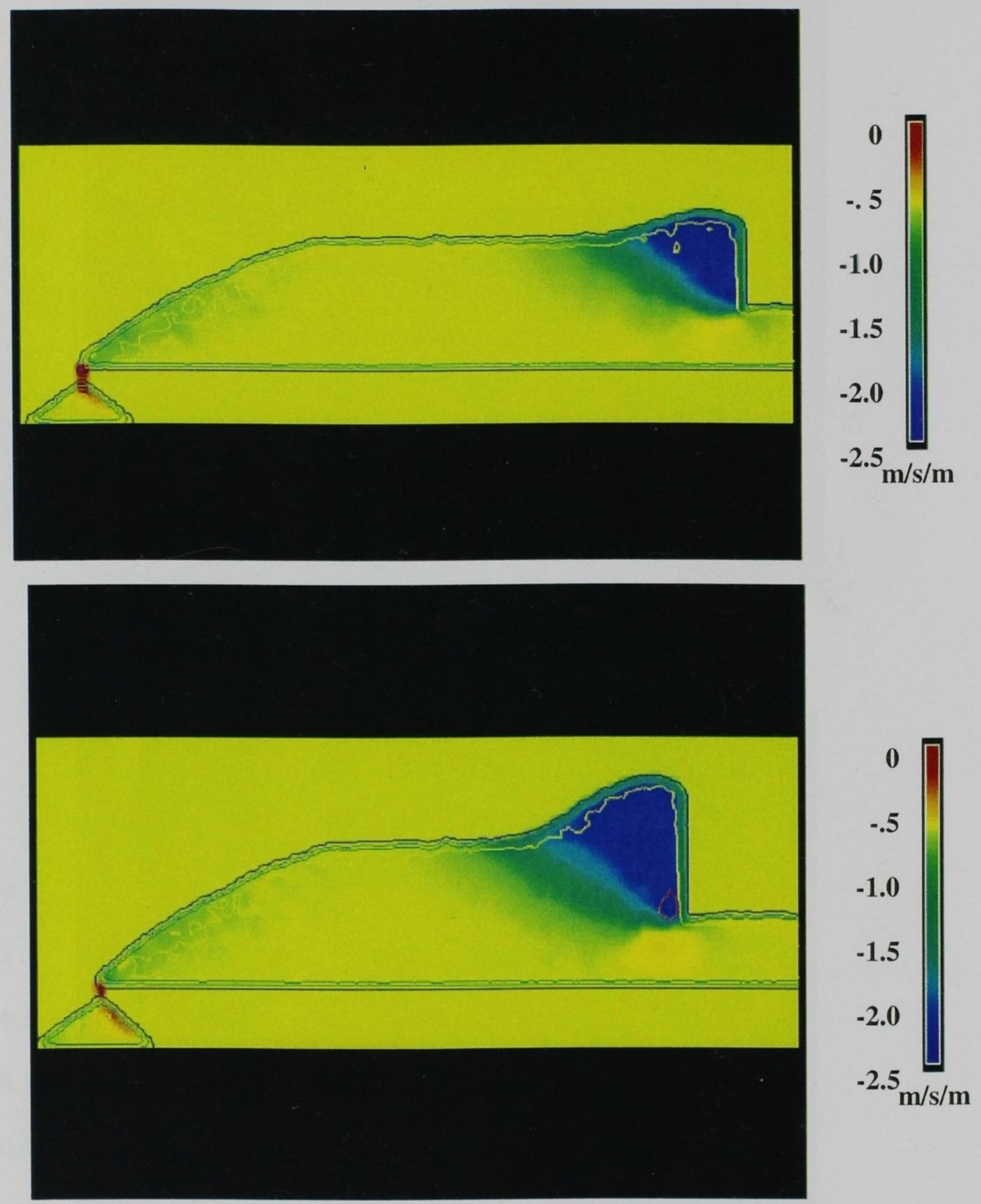

Figure 4.14: Rotation Rate at (a) $1 \mathrm{~cm}$ of Plowing (b) $2 \mathrm{~cm}$ of Plowing 

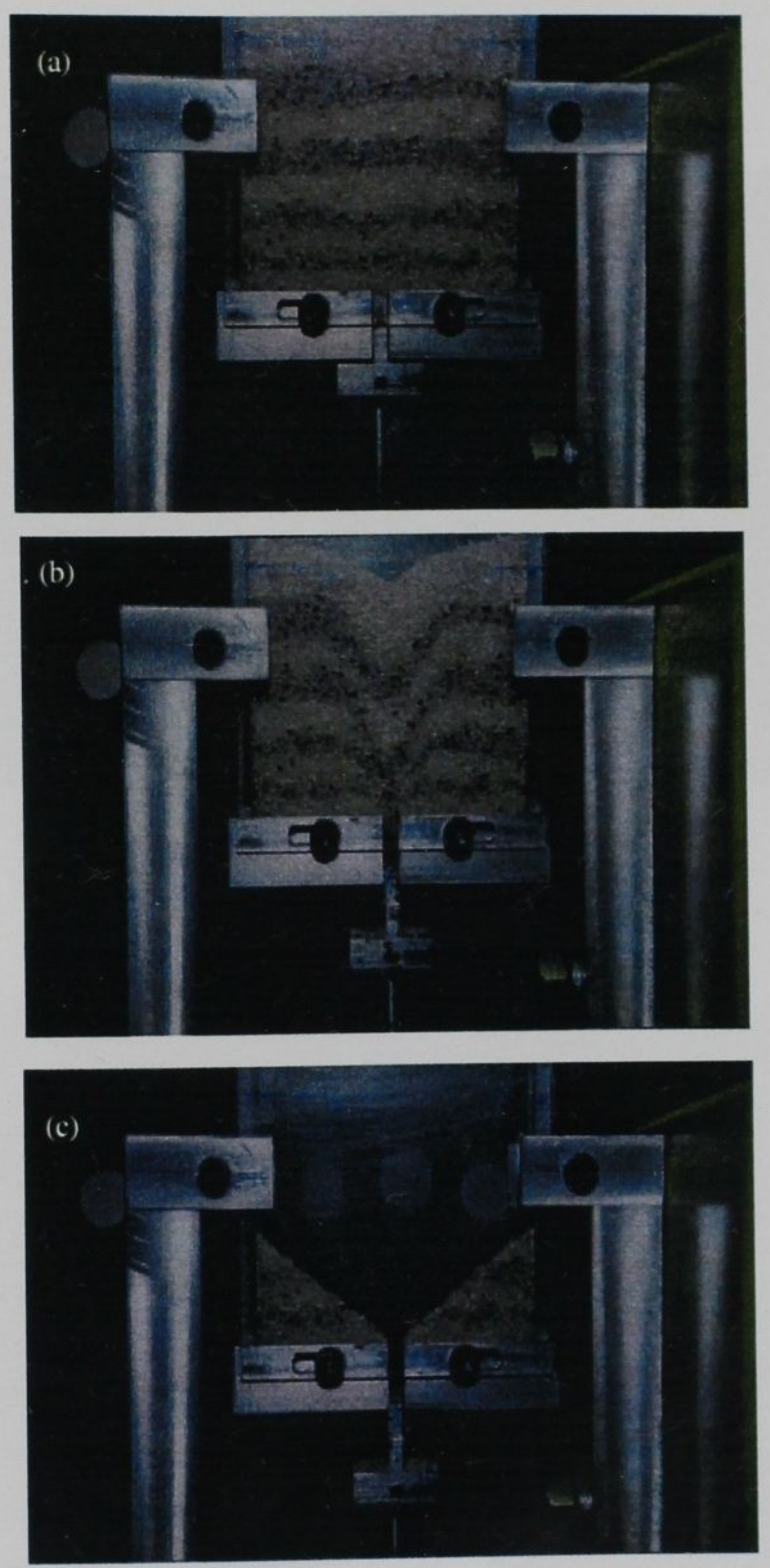

Figure 4.15: Trap Door Problem 

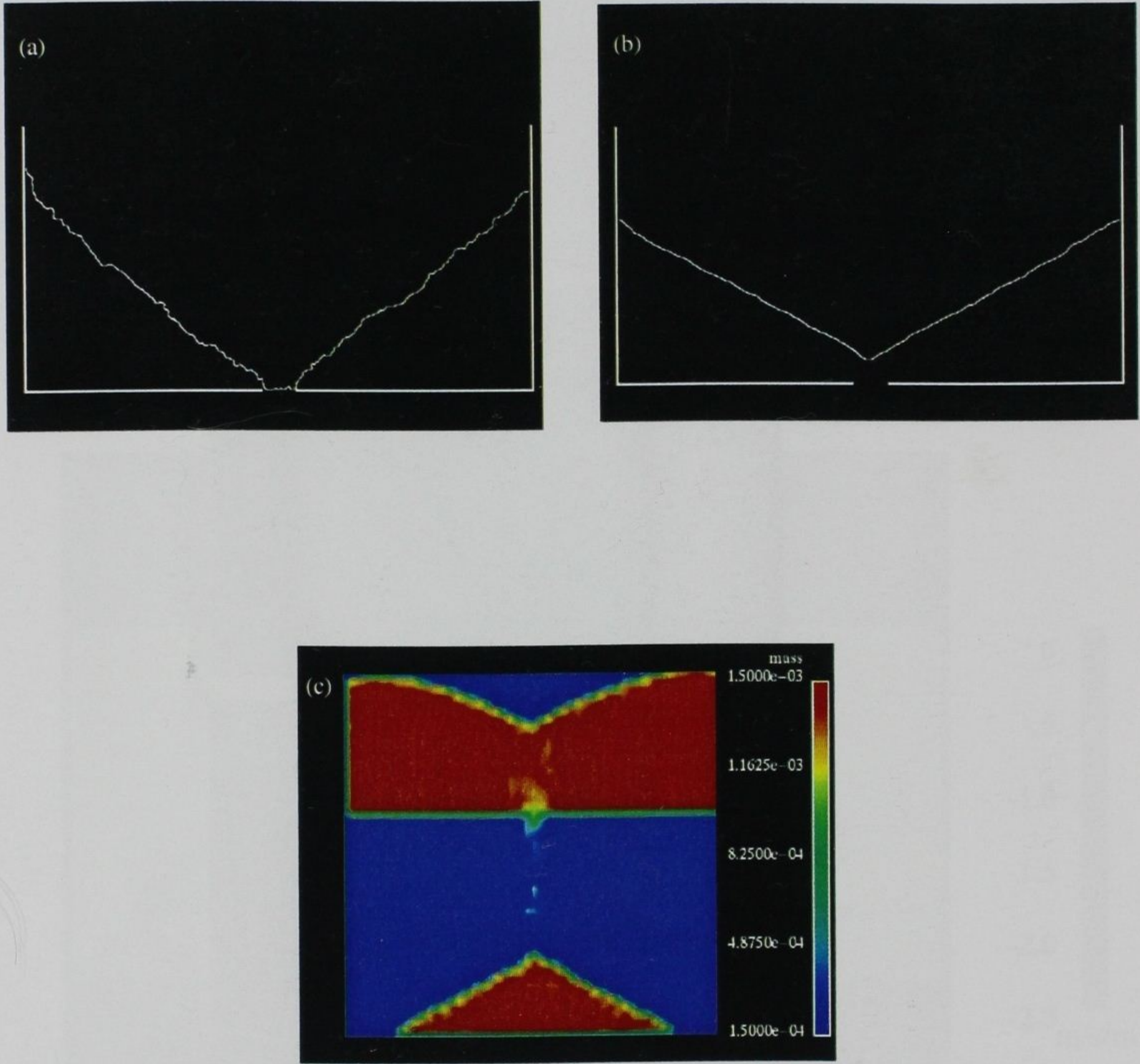

Figure 4.16: Surface Profile at 2 Seconds for (a) Ottawa 20-30 (b) Glass Beads (c) Simulation 


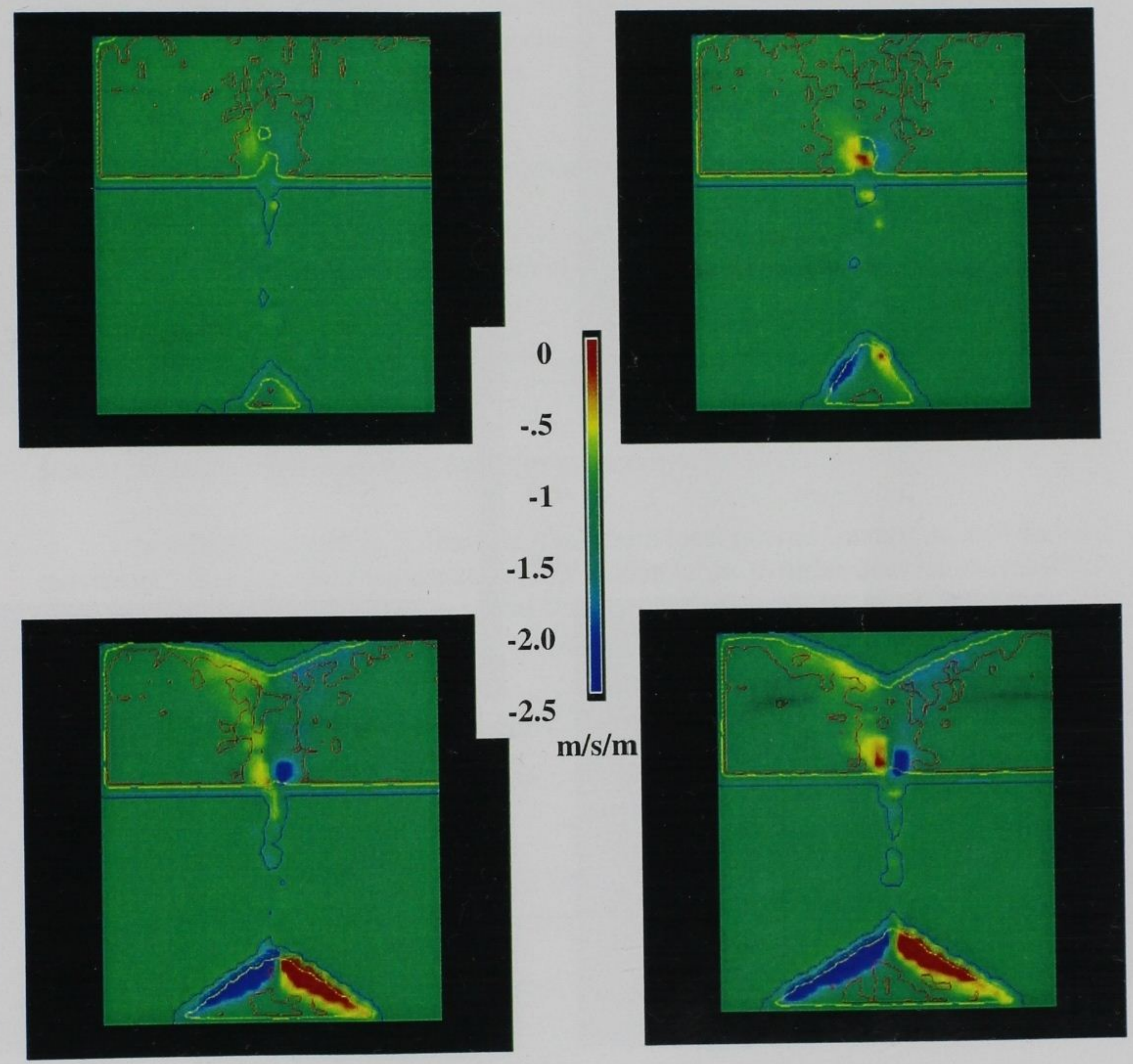

Figure 4.17: Horizontal Velocity Gradient with Respect to y at (a) 0.5 Seconds (b) 1.0 Seconds (c) 1.5 Seconds (d) 2.0 Seconds 

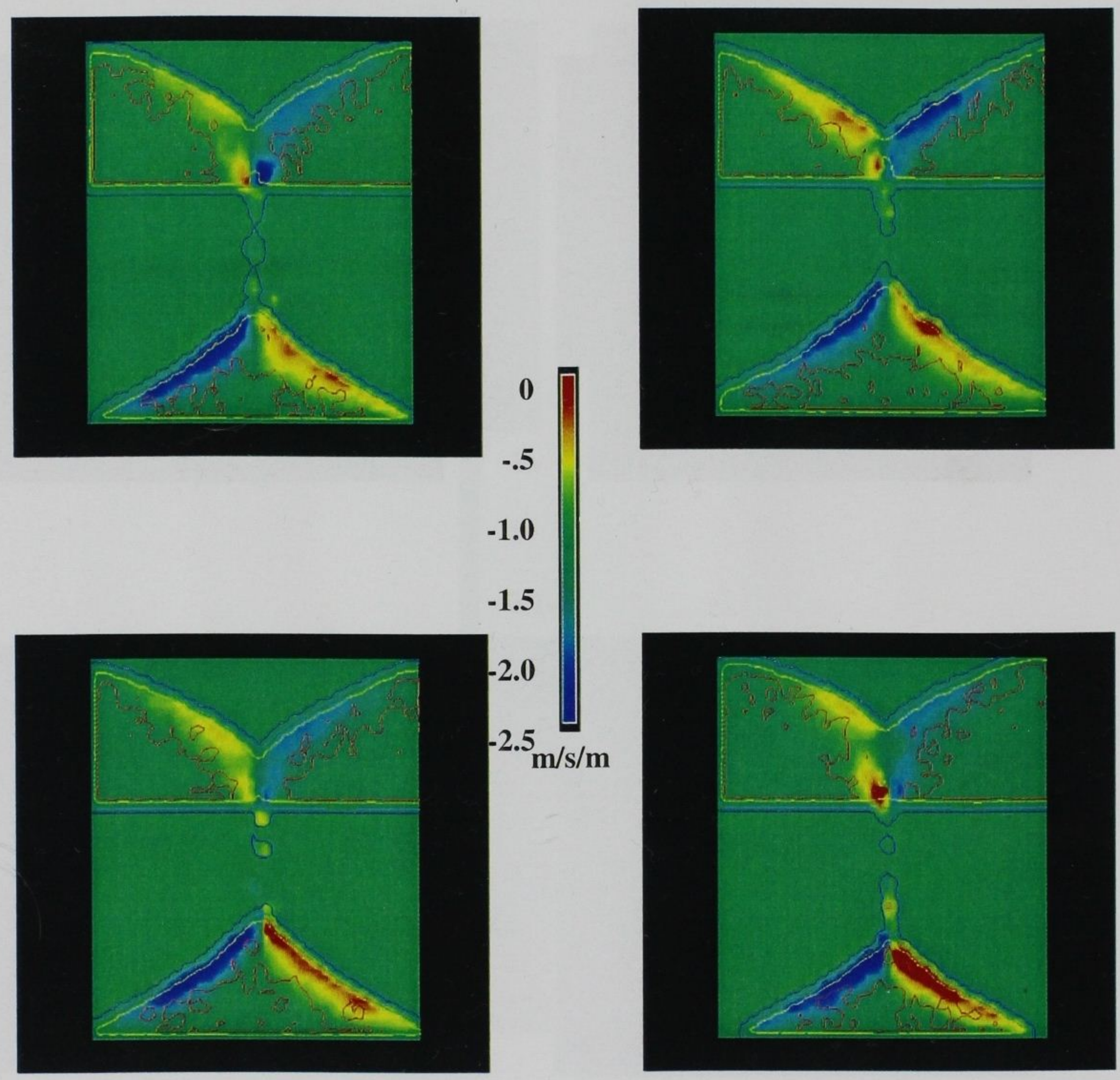

Figure 4.18: Horizontal Velocity Gradient with Respect to y at (a) 2.5 Seconds (b) 3.0 Seconds (c) 3.5 Seconds (d) 4.0 Seconds 


\section{CHAPTER V}

\section{Large Deformation Constitutive Law}

In Chapter 3, an averaging process using the concept of an implied averaging volume was described. This averaging technique is a methodology for obtaining continuum properties of a discrete system. In this chapter the descriptive averaging process is applied to the particle data from the soil plowing simulation described in Chapter 4. From this analysis a large deformation constitutive law for the smoothed continuum is developed based on a statistical description of the DEM particulate system.

\subsection{Governing Equations for Smoothed Particle System}

By viewing the particulate system as a continuum, the equation of motion for any material point within the continuum is written as:

$$
\frac{\partial \sigma_{i j}}{\partial x_{j}}=-\rho b_{i}+\rho \frac{\partial v_{i}}{\partial t}
$$

where $\rho b_{i}$ is the distributed body forces (e.g., gravity).

In a DEM simulation, forces that result from local particle contacts control the particle movement. In a smoothed representation, the motion of the particles does not necessarily obey the equation of motion. Rather the spatial average of the contact forces satisfies the equation of motion. Using the method of weighted residuals to average the equation of motion over a region, the following equation holds:

$$
\int_{R} M\left(x-x^{\prime}\right)\left[\frac{\partial \sigma_{i j}}{\partial x_{j}}+\rho b_{i}-\rho \frac{\partial v_{i}}{\partial t}\right] d y=0
$$

where $M\left(x-x^{\prime}\right)$ is a positive weighting function that weights the influence of a particle, at location $x$, as a function of its distance from the averaging point $x^{\prime}$.

By rearranging parts of the equation and applying integration by parts [55] to the stress gradient, the equation may be written as:

$$
\int_{R}-\frac{\partial M}{\partial x_{j}^{\prime}} \sigma_{i j} d x^{\prime}=\int_{R} M\left(x-x^{\prime}\right)\left[-\rho b_{i}+\rho \frac{\partial v_{i}}{\partial t}\right] d x^{\prime}+\int_{s} M \sigma_{i j} n_{j} d S
$$

This form of the equation is advantageous because the stress does not have to be continuous. The integration by parts produces the surface integral, which can be viewed as the traction 
boundary condition satisfied in a weighted sense. For the remainder of this discussion it will be assumed that sampling is being taken away from a boundary. Applying the mean-value theorem to the right-hand side of Equation (5.3) yields:

$$
M\left(x-\bar{x}^{\prime}\right) \int\left[-\rho b_{i}+\rho \frac{\partial v_{i}}{\partial t}\right] d V
$$

where $d V$ represents the volume of the region. If the region is small, the value of the integral can be approximated as the product of the average velocity gradient and the volume of the region:

$$
M\left(x-\bar{x}^{\prime}\right) \overline{\left[-\rho b_{i}+p \frac{\partial v_{i}}{\partial t}\right]} \Delta V .
$$

A similar procedure can be performed to the left-hand side of equation (5.3) to yield:

$$
\sigma_{i j} \frac{\partial M}{\partial \overline{x^{\prime}}{ }_{j}}=M\left(x-y^{\prime}\right) \overline{\left[-\rho b_{i}+\rho \frac{\partial v}{\partial t}\right]} .
$$

Equation (5.6) implies that the equation of motion for the system can be satisfied, on the average, by using a sampling of the particle interactions in view of

$$
\bar{\sigma}_{i j}=\sum \phi^{k} f_{i}^{k} n_{j}^{k}
$$

In the DEM simulations, the average particle stress is computed by summing the $k$ contact force vectors, $f_{i}$, around the particle

$$
\bar{\sigma}_{i j}=\frac{l}{V} \sum_{i=1}^{k} f_{i} n_{j}
$$

where $l$ is the average distance between particle centers that are in contact, $V$ is the volume of the particle, and $n_{j}$ is the contact normal vector.

If the coordination number, $\mathrm{C}$, is known then Equation (5.8) can be written as

$$
\bar{\sigma}_{i j}=\frac{1}{V} C \overline{f_{i} n_{j}}
$$

where $\overline{f_{i} n_{j}}$ is the average force vector acting on the particle. 
Equation (5.9) implies that the macroscopic behavior of the discrete system could be approximated from a sampling of the contacts. This observation suggests that it could be possible to replace the highly detailed discrete particle system with a simpler model that represented an average particle. The average particle would maintain the same average properties of the total discrete system.

The development of the smoothed particle constitutive law requires that a statistical description of the coordination number and the $\overline{f_{i} n_{j}}$ be developed.

\subsection{Analysis of DEM Plowing Simulation}

\subsubsection{Material Points}

Using the plowing simulation described in Chapter 4 , four material points were established around the plow face to monitor particle contact information during the plowing. The material point was defined as a point in space where descriptive averaging of the surrounding particle data was performed. A sampling radius of $2 \mathrm{~mm}$ was used. The material point moved with the average velocity field as computed for the surrounding particles. The velocity of a material point is determined using Equation (3.13). These material points provide a time history of each state variable (i.e. stress, strain) as related to the initial configuration of the material. Results from the plowing simulation were used to examine the effects of sampling size on calculation of state variables following the procedure of Chapter 3. Figure 5.1 shows the initial and final location of the four material points during the first 5-mm of plowing. Each point is labeled based on its initial coordinates in the $\mathrm{x}$ and $\mathrm{y}$ direction. The points were labeled using the notation of xxyy. Where $\mathrm{xx}$ is the initial location of the point in $\mathrm{mm}$ in the $\mathrm{x}$ direction and yy is the initial location in $y$. The four points are $9811,9310,9515,9505$.

Point 9310 was also placed in the shear zone, but farther away from the blade. Point 9515 was placed above the shear zone, in the region where motions were predominantly rigidbody-motions. Point 9505 was placed below the shear zone and was established as a point that had little or no movement associated with it. Each of these four sampling points exhibited significantly different loading characteristics, and it was expected that they would provide the range of information required to develop a generalized macroscopic model of the granular system.

Table 5.1: Sampling Sizes for Ottawa 20-30

Sampling Radius, h Approximate

(mm)

1

2

3

5
Number of Particles in Sample

30

250

820

3800 


\subsubsection{Sample Size}

Only material point 9811 was used to demonstrate the effects of sampling size. Four characteristic kernel lengths were used in this examination. The four sampling radii and approximate number of particles in the samples are shown in Table 5.1.

Lagrangian strain was computed for the material point by methods described in Section 3.2.4, and the Cauchy stress was computed using Equation (3.40). Figure 5.2 shows the change in the Jacobian determinant, which is a measure of relative volume change, as a function of shear strain $E_{12}$, for the four sampling sizes over the first $5 \mathrm{~mm}$ of plowing. The first observation is that the shear strain decreased as the sampling sized increased. This can be explained by observing that, as shown in Figure 5.3, for a condition of an abrupt shearing face, the change in horizontal velocity with respect to the vertical axis will decrease as the vertical length is increased. This illustrates the point that if the sampling size is set too large, localized failure phenomenon will be lost. The other interesting observation from Figure 5.2 is that all cases except the largest sampling size show the volume contracting. The three smaller sampling sizes all showed the same trend of volume contraction with increased shear strain. The largest sampling size differed from the others in that after an initial volume contraction the volume began to expand. It is concluded that, at the largest sampling size, the dilation zone above the shear zone is influencing the results. Some of the contraction is from the compression of the soft particles. It is expected that the particle compression would be fairly constant along the face of the plow. So it is expected that the effects of sampling size in this region would be significantly affected by particle compression.

Figure 5.4 plots the change in Cauchy shear stress, $\sigma_{12}$, as a function of Lagrangian shear strain. As expected, the maximum shear stress increased as the sampling size decreased. The two middle-sampling sizes have similar trends.

It is not the intent of Figure 5.4 to suggest that there is a relationship between the Cauchy shear stress and the Lagrangian shear strain. As shown in Figure 5.5 the Cauchy stress element is measured from the spatial coordinate system, while the Lagrangian shear strain is measured from the material coordinate system, which deforms in the view of a spatial observer. The use of the Lagrangian shear strain in Figure 5.4 is simply a convenient parameter to measure the progress of the system.

\subsubsection{Distribution of Particle Contacts and Forces}

By obtaining the spatial velocity gradient, $L_{i j}$, as described in Chapter 3, the average deformation field can be developed for a location within the granular media. Figure 5.6 shows the distortion of a unit square centered around Point 9811 during the first 5 -mm of plowing. It can be seen that the material at Point 9811 is compressing in the horizontal direction, extending in the vertical direction, and shearing. 
Sampling the contact data provides meaningful macroscopic information about the behavior of the granular media under extremely large deformation. Figure 5.7 shows the distribution of contact normal forces for all the contacts in the sampling region of Point 9811 at $5 \mathrm{~mm}$ of plowing. From this figure, it can be concluded that the force distribution for the granular material exhibits a large, random component. This should be expected because the granular material does not move with affine motions; but, in fact its motions are nonaffine and, for a region to exhibit a shear, particles must slide and move over each other.

To provide a statistical view of the data, the data were sorted in equally spaced bins, based on contact angles. Thirty-degree contact angle bins were arbitrarily selected for sampling the particle contact data, as shown in Figure 5.8.

Figure 5.9 plots the probability of occurrence of normal contact forces sorted by contact angle increment bins for plowing at $5 \mathrm{~mm}$. By examining the normal contact force in terms of the bins, a trend from the force data begins to appear as shown in Figure 5.9. The area under each of these curves would represent the average force for that particular bin of contact. Figure 5.10 shows the force data from Figure 5.9 in percentiles. It clearly illustrates that the largest average normal forces are occurring in the 0 to 30 -degree contact region.

Figure 5.11 shows the average contact force for each contact angle increment bin during plowing. From this figure, it is seen that the average force data quantitatively follows the trend of the smoothed deformations shown in Figure 5.6 in that the higher average forces are occurring in the horizontal direction where the maximum compression is occurring.

\subsubsection{Estimate of Coordination Number}

Several researchers have developed relationships between the coordination number and void ratio, based on experimental data. Chang et al. [10] presented several of these relationships. Figure 5.12 presents the coordination number as a function of void ratio for the results obtained from DEM simulation. Additionally, experimental data points from other researchers [30], [38], and [54], presented by Chang are shown in Figure 5.12. In general, the data obtained from the Discrete Element Model was similar to that obtained from experimental data. The DEM data tended to have a lower coordination number than that of the experimental data.

Analysis of the DEM has suggested that the mean stress may be a better predictor of the coordination number. Figure 5.13 shows the relationship of average coordination number with the mean stress of various material points during the plowing DEM simulation. The material points were selected in the regions just in front of the plow. For this simulation, the average coordination number can be predicted reasonably well by the mean stress of the material point. The data from the four sampling points shown in Figure 5.13 was fitted with both linear and non-linear regression. The linear regression yielded the best fit.

$$
C=6.41-0.00021 \bar{\sigma}
$$


(a)

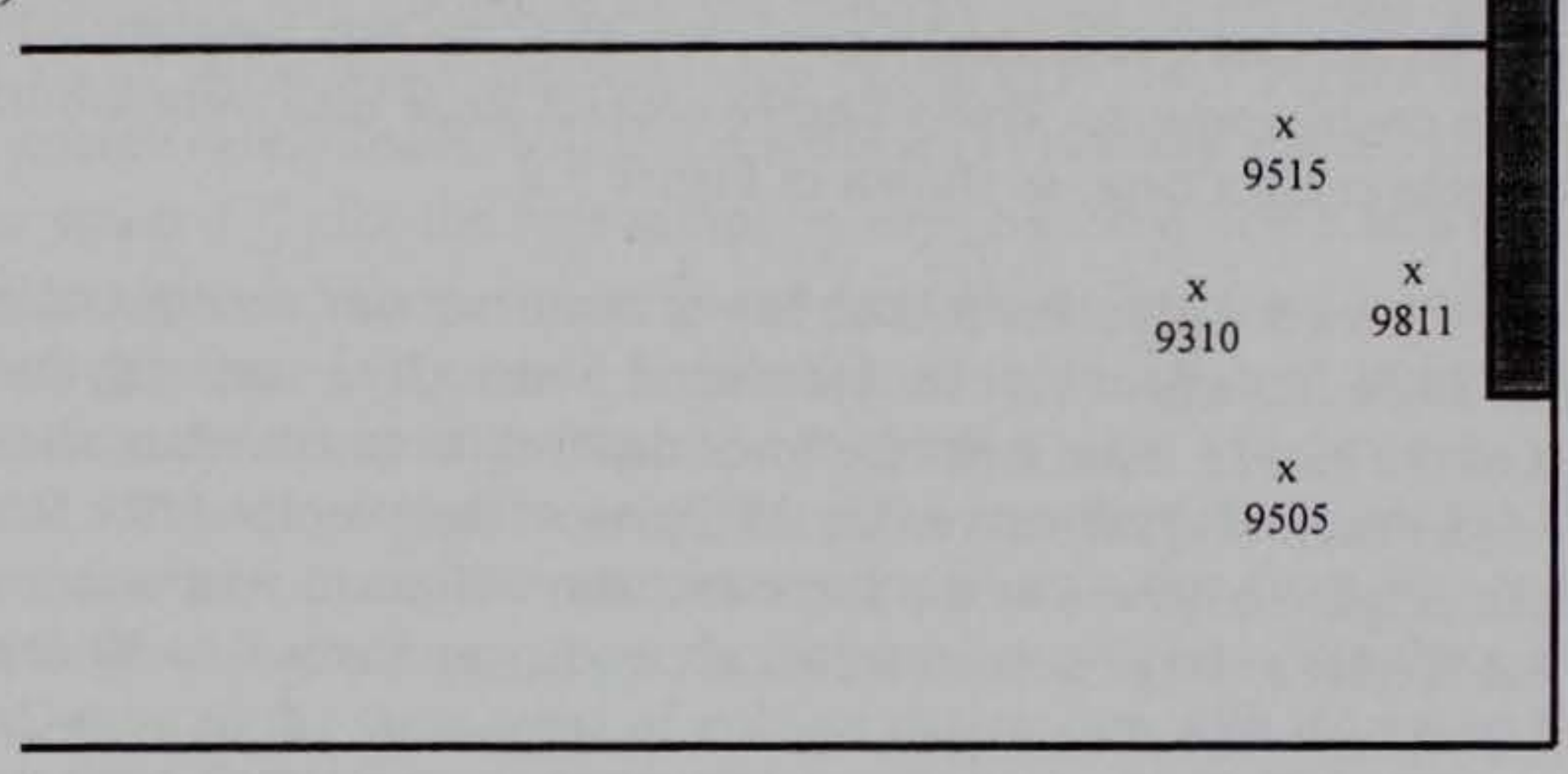

(b)

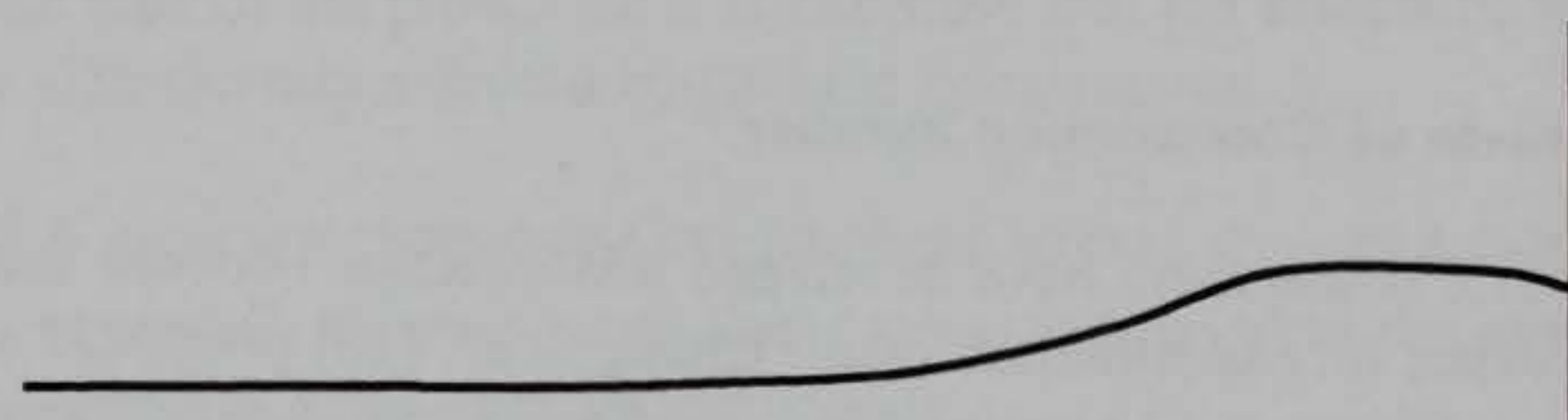

$\mathrm{x}$

9515

x $\quad x$

9310

9811

Figure 5.1: Initial Location of Material Points 


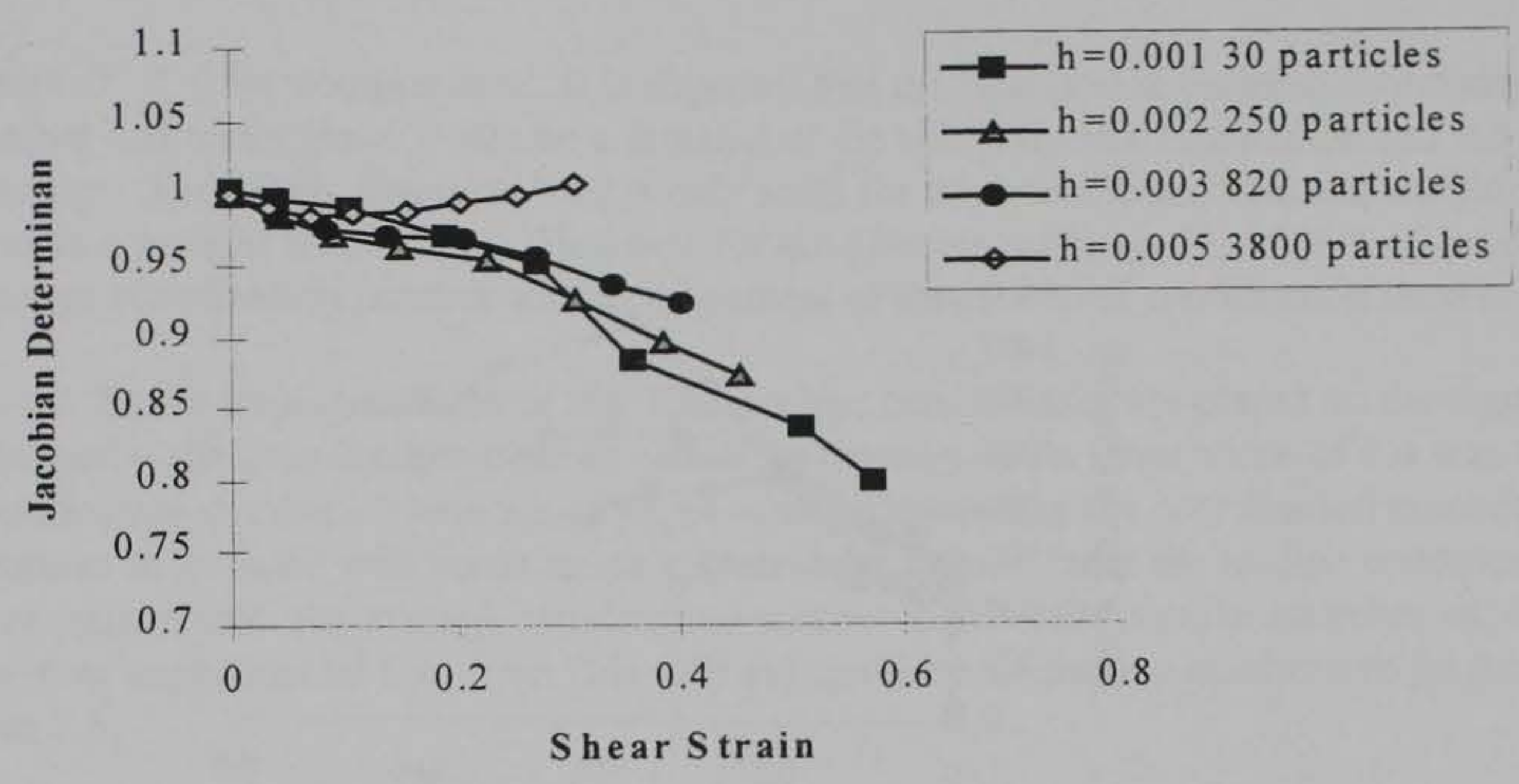

Figure 5.2: Effects of Sample on Jacobian Determinant

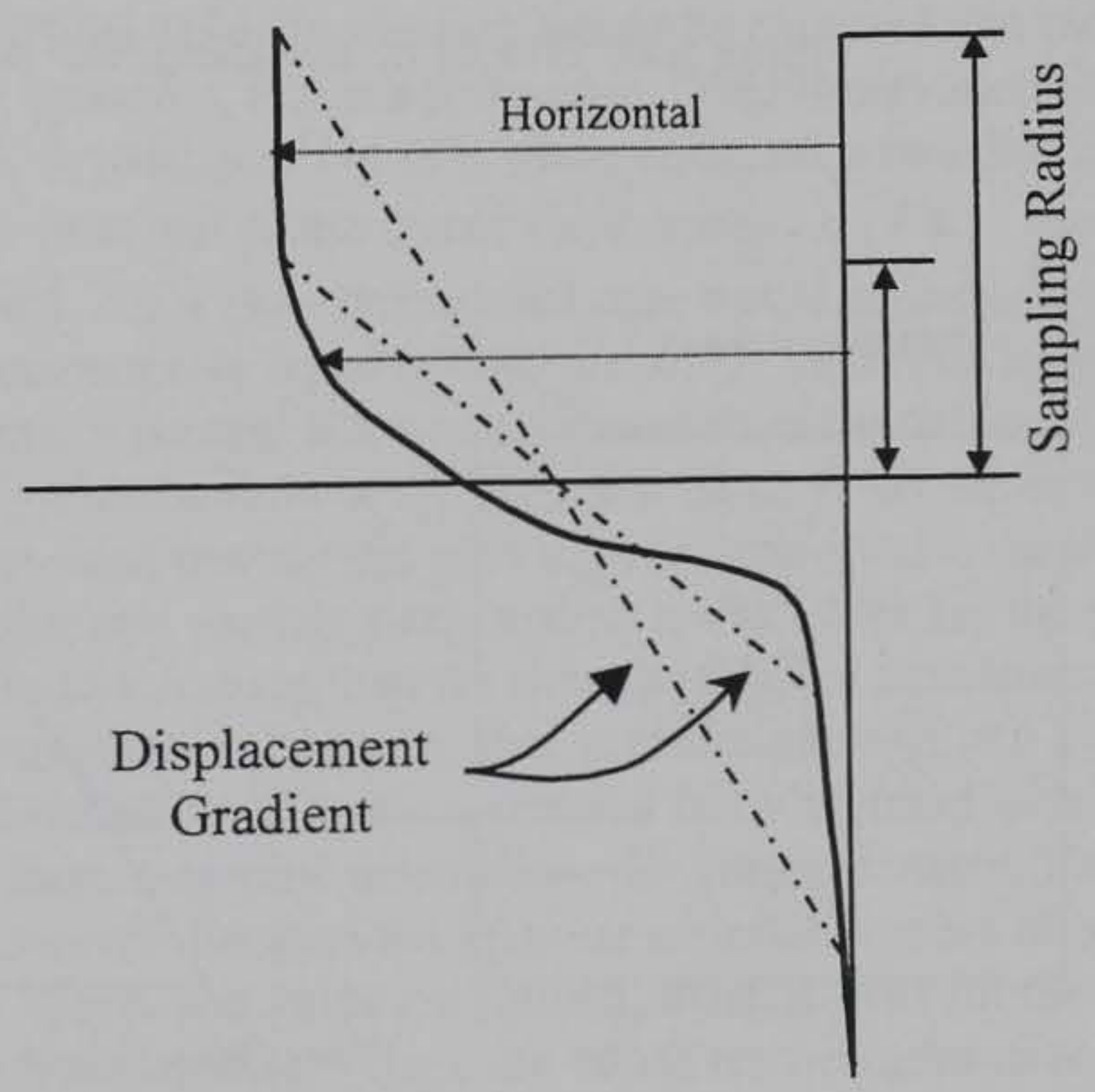

Figure 5.3: Effects of Sampling Size on Shear Strain 


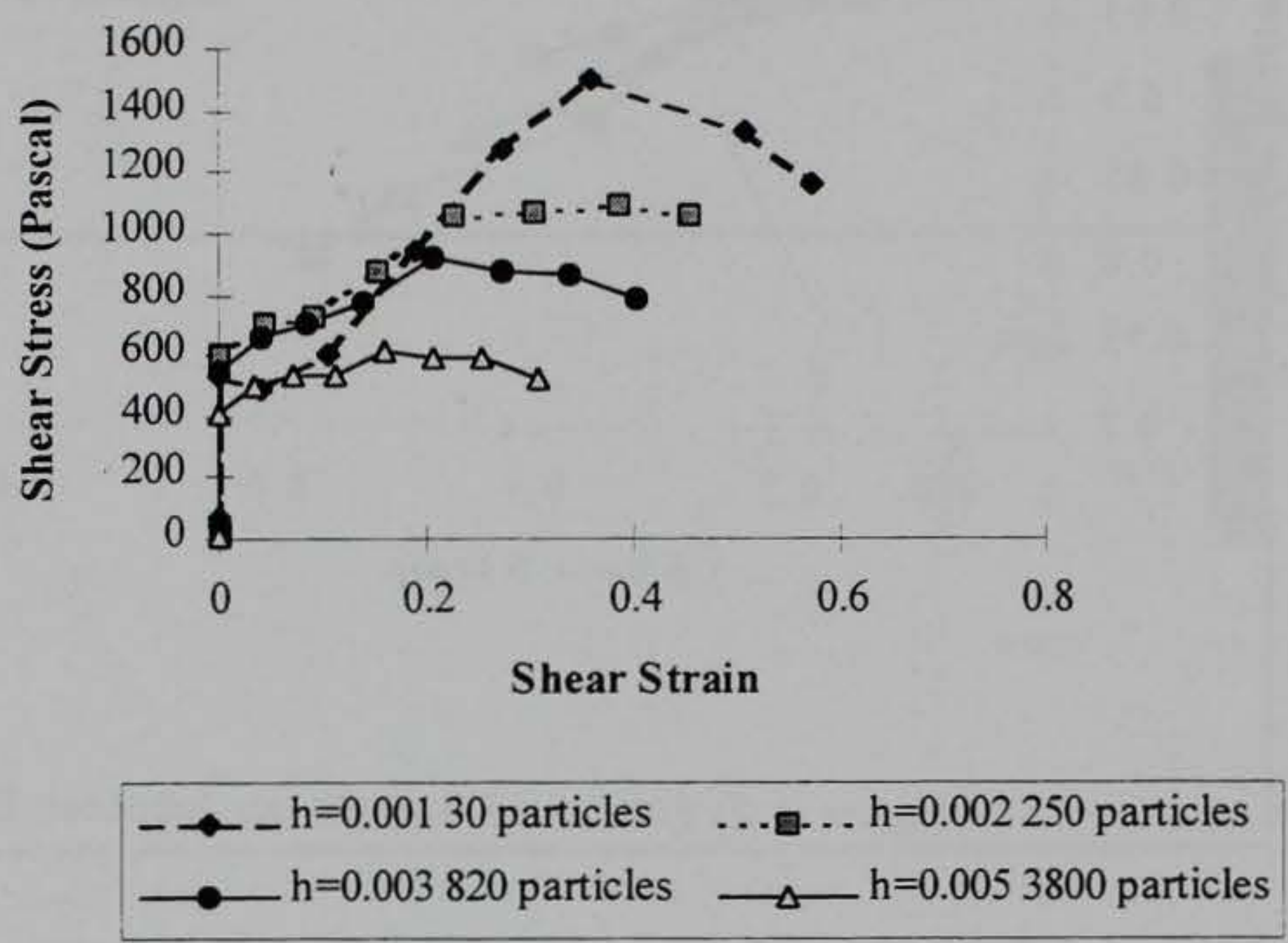

Figure 5.4: Effects of Sampling Size on Shear Stress

Undeformed Shape

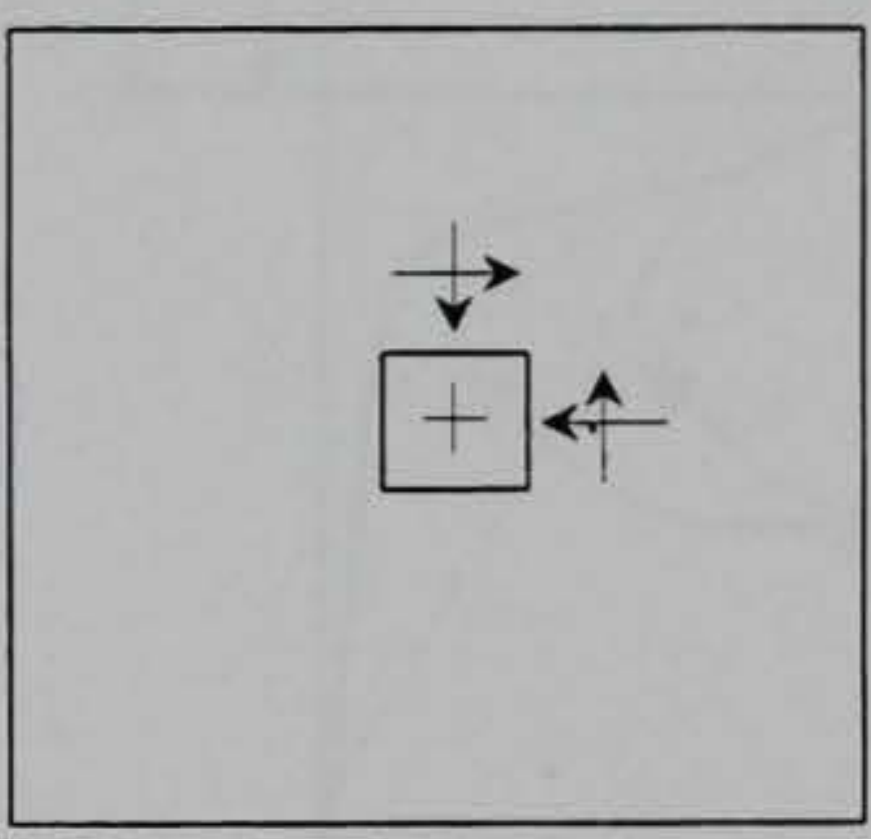

Deformed Shape

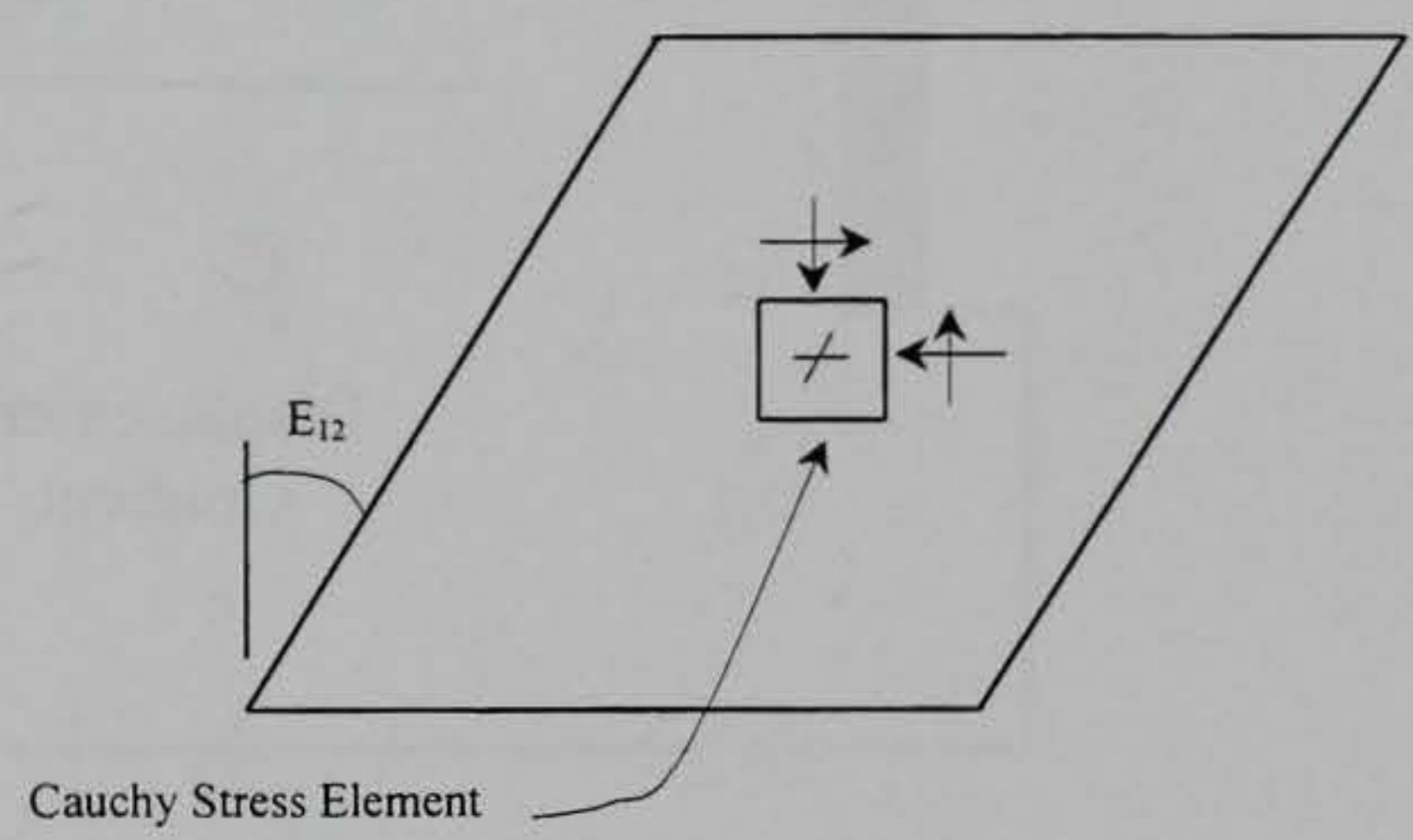

Figure 5.5: Spatial and Material Coordinate Systems 
where $\bar{\sigma}<0$ for compression. It is expected that the relationship between coordination number and mean stress would be a function of the soil grain size distribution and the initial porosity. Therefore, Equation 5.10 is only valid for this specific problem and should not be used as a general relationship. However for the plowing problem, Equation 5.10 predicted the average coordination number within 3.5 percent of the measured coordination number.

In the implementation of this relationship, certain limits are placed on the equation. Obviously, the coordination number cannot be negative and a lower value of 6.4 was set for the coordination number. There are physical considerations why the coordination number cannot continue to increase with linear stress and an upper limit. Under the loading conditions of the plow experiment, the average coordination number in the DEM simulation never exceeded 7.5, however experimental data from Oda [39] suggest the coordination number can go much higher than 7.5.

\subsubsection{Average Normal Vector}

An examination of the distribution of contact angles within the plowing simulation revealed that the contact angles are uniformly distributed, although the forces being transmitted are not. Figure 5.14 shows the distribution of contact angles at different amounts of plowing for the material point described in the previous section. What is interesting from this figure is that the distribution of contact angles remains essentially uniform even over large strains. If the granular material moved with affine motions, it would be expected that there would develop a preferred contact angle; however, this is not the case. This observation has been presented by other DEM simulations, in particular $\mathrm{Ng}$ [37] who performed a two-dimensional shearing test in which the ratio of the principal fabric tensor components, $n_{11} / n_{22}$, reached an upper limit of 1.2. This upper limit of 1.2 in a two-dimensional case would be the equivalent of having the principal fabric tensor components equal to 0.45 and 0.55 , these values correspond to distortional strain of about 5 percent if it was attributed to affine deformation. The results from the plowing simulation yielded a ratio of the principal fabric tensor to be less than 1.2. However, it should be pointed out that the plowing simulation was a three-dimensional simulation, using five different particle sizes, and so it was easier for the particles to arrange themselves in a more random fashion than the two-dimensional simulation of $\mathrm{Ng}$. The ultimate observation of both simulations is, however, that granular material will tend to reorient its contacts such that the distribution of contacts remains fairly uniform with direction. If the contact angles were to favor a specific orientation, the granular material itself would have to deform like rubber. However, because the granular material consists of essentially rigid objects, when shearing occurs and voids are formed, material will fill the voids and the uniform distribution of contacts is maintained. Thus, the fabric tensor varies in accordance with smoothed deformation at small distortions, but reaches a limiting value at a few percent strains. 


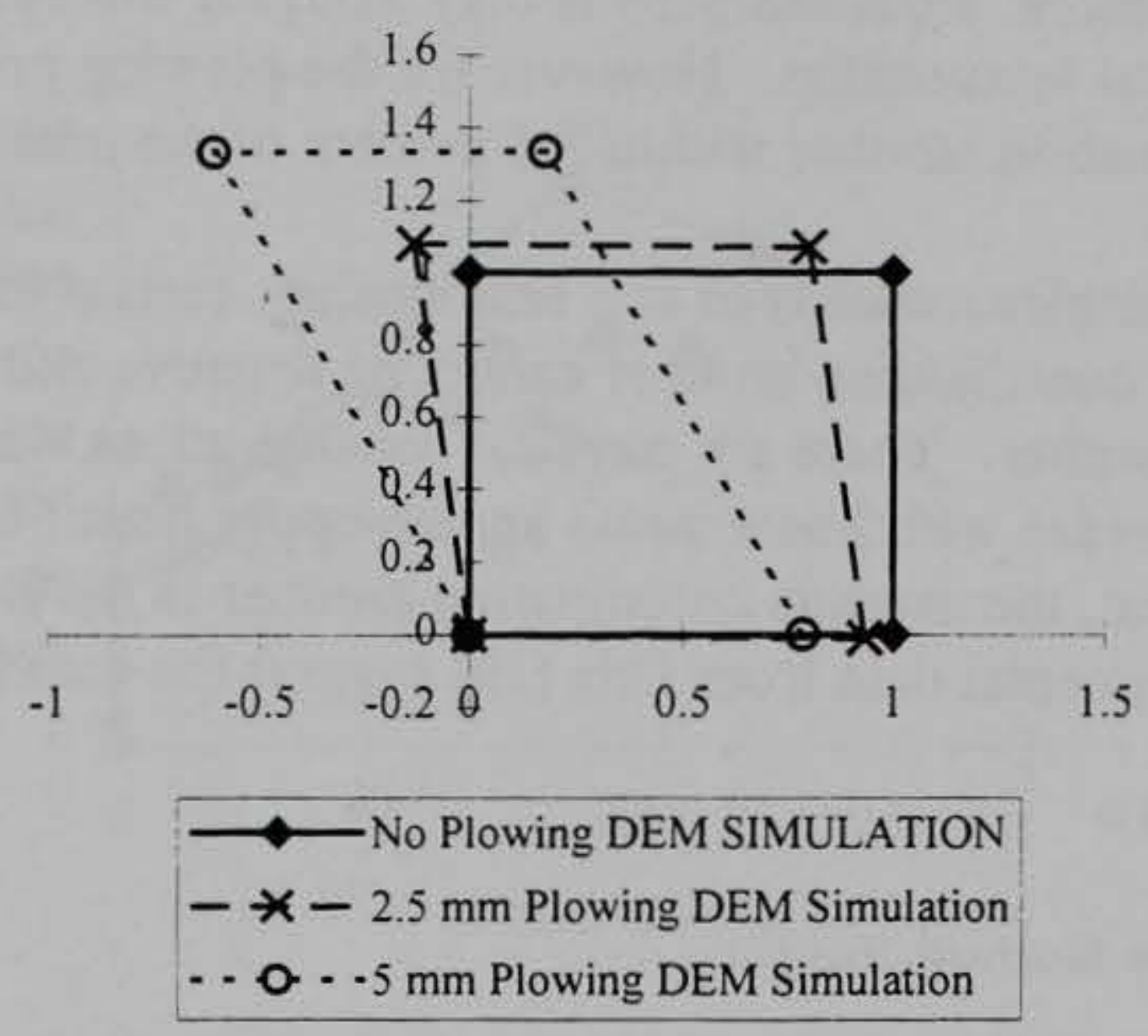

Figure 5.6: Smooth Deformations for Sampling Point 9881

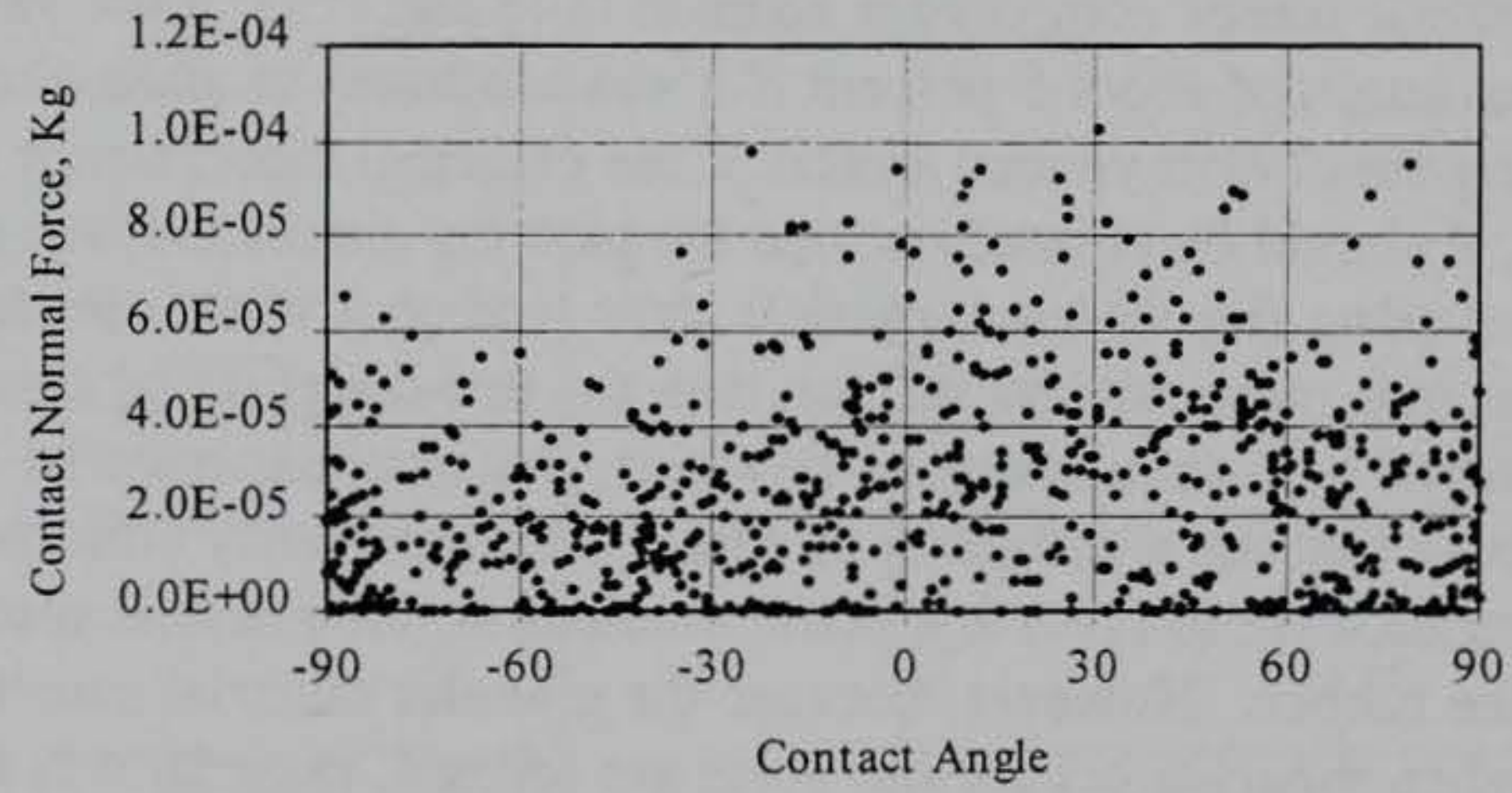

Figure 5.7: Distribution of Normal Forces by Contact Angle at $5 \mathrm{~mm}$ Plowing 
4 Direction of Plowing
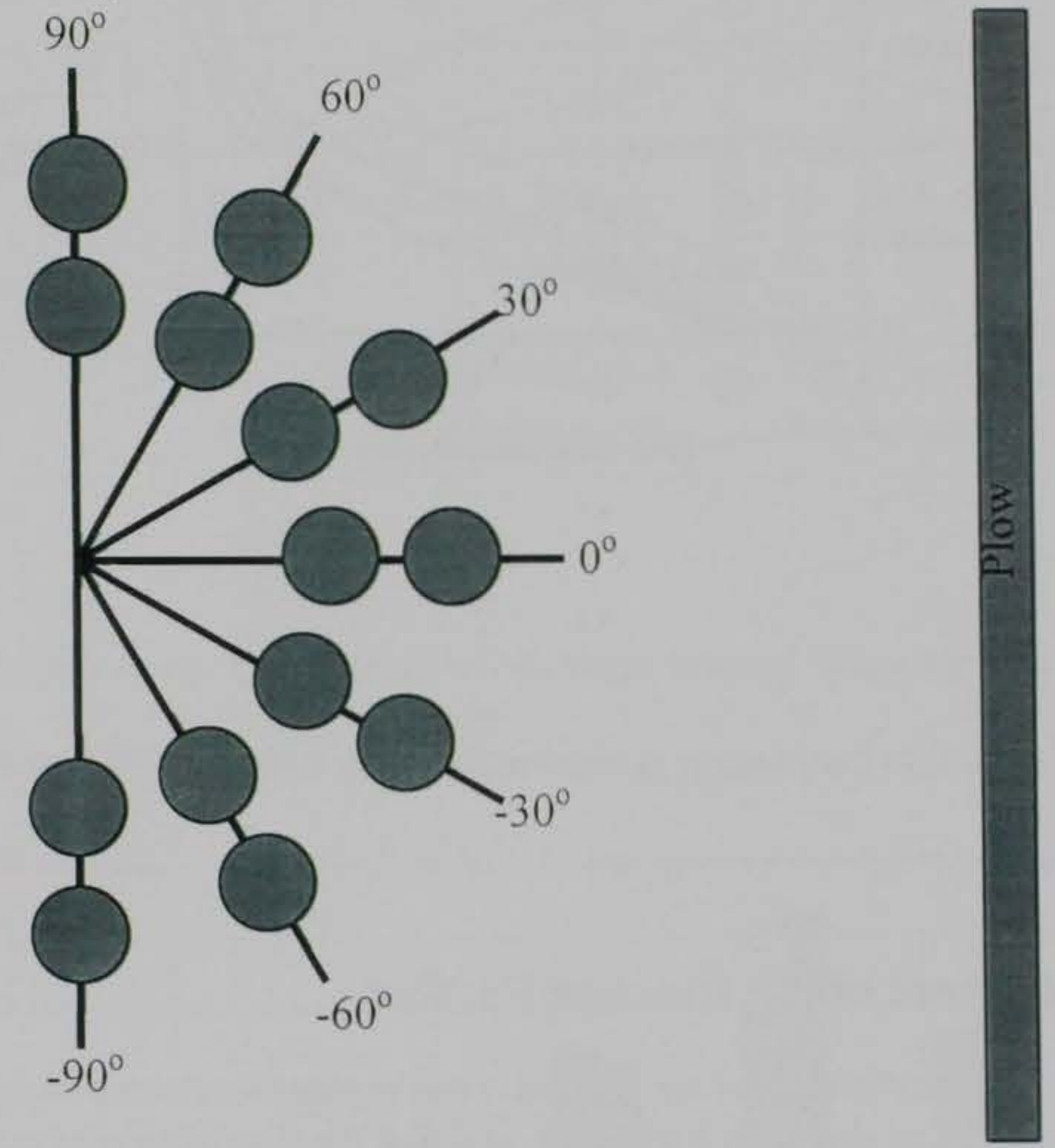

Particle Contact Angle

Figure 5.8: Contact Angle Sampling Bins 


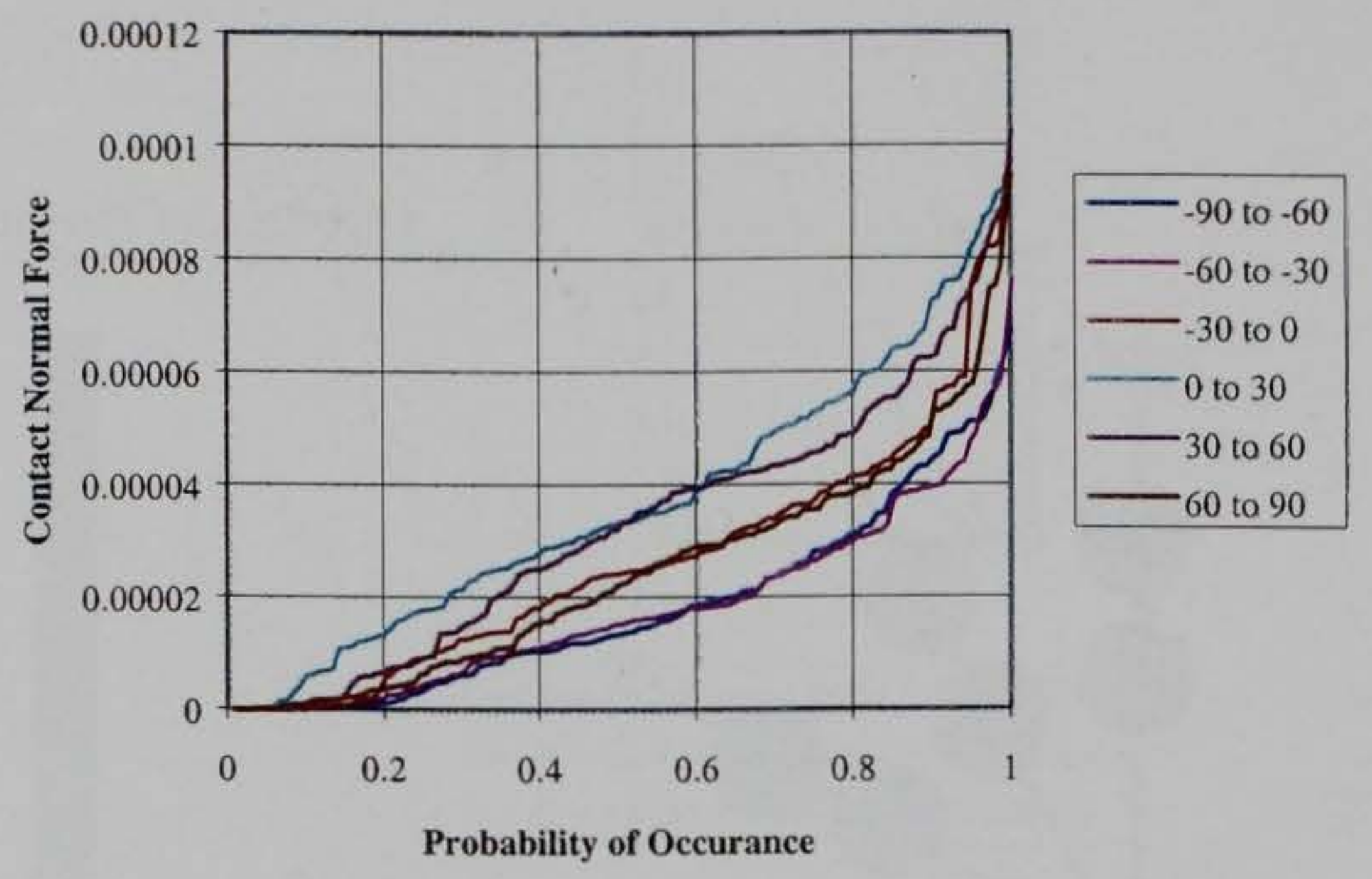

Figure 5.9: Distribution of Normal Force by Sampling Bins at $5 \mathrm{~mm}$ Plowing

\subsection{Development of the Average Particle}

Based on the fact that force data are derived when contact forces are averaged over a fixed contact angle space as shown in Figure 5.9, and that the distribution of contact angles is uniformly distributed during the simulation, one representation of the average granular system could be that of a spoke system as shown in Figure 5.15. If the sampling of the DEM particles is sufficiently large, it follows that an average particle could be represented as having equally spaced contacts as represented by the spoke model. The forces at the contacts can be defined from the deformation that occurs as a result of the smoothed velocity field as computed by the spatial velocity gradient. The smoothed velocity field for the average particle can be computed from the estimate of the current strain rate as described by Equation (3.25). The relative incremental displacement for a contact direction is computed as:

$$
\delta_{l}^{r}=\Delta t E_{I J} n_{J} l
$$

where $n_{J}$ is the contact vector in the initial (material) configuration and $l$ is an average distance between centers of particle contact pairs. 


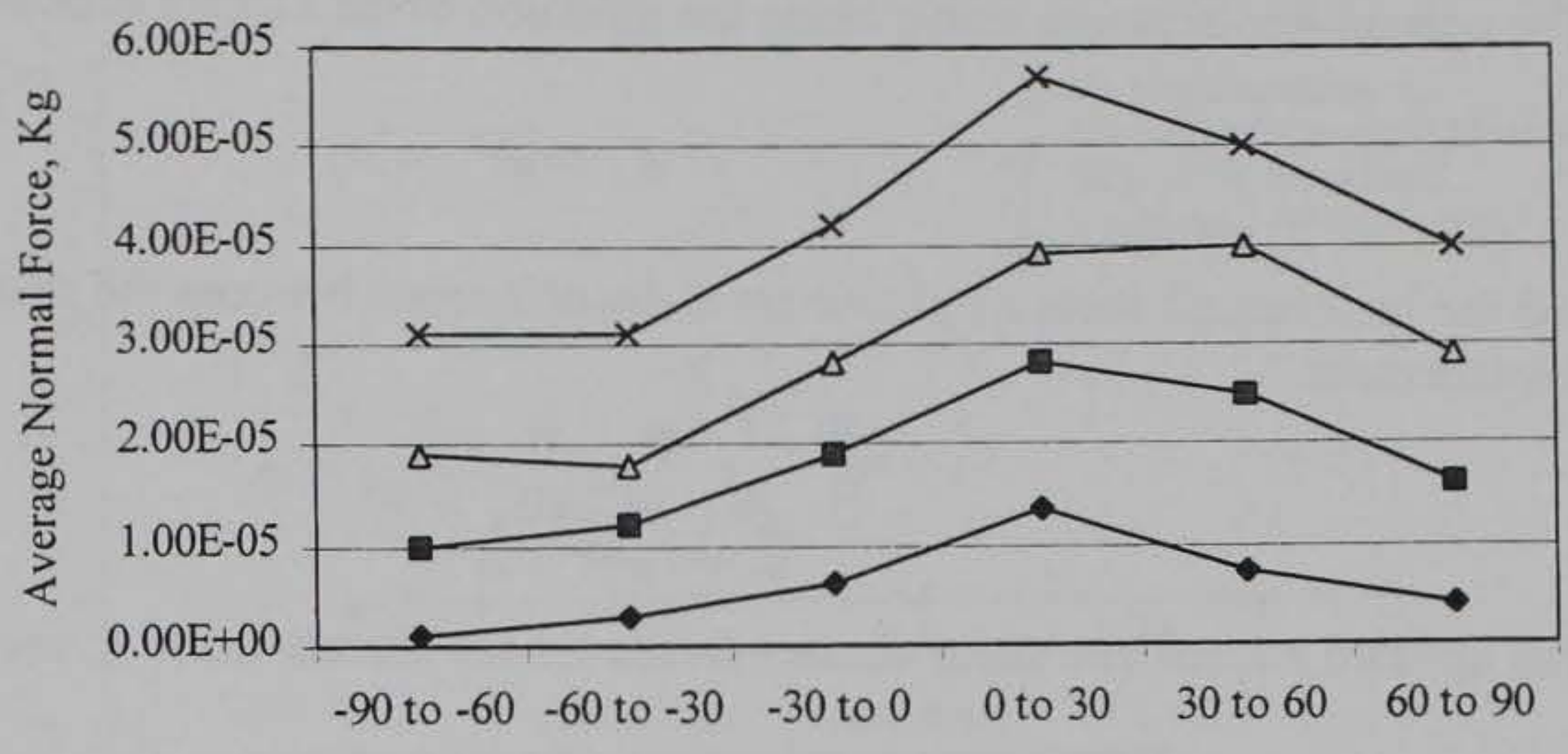

Contact Angle Bin

$\rightarrow$ 20th Percentile $\rightarrow$ 40th Percentile
$\rightarrow$ 60th Percentile $\rightarrow$ 80th Percentile

Figure 5.10: Distribution of Average Normal Force by Percentile

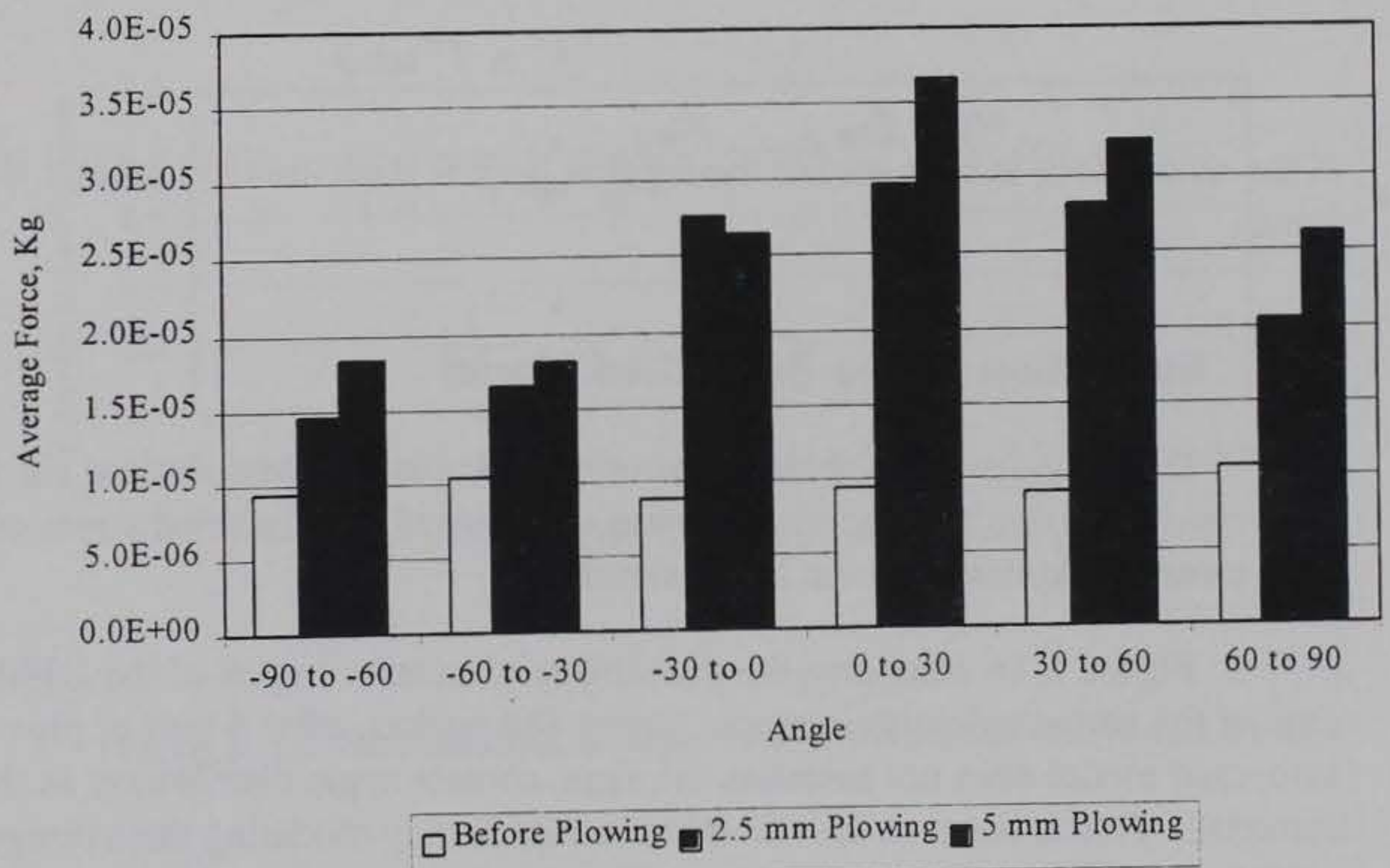

Figure 5.11: Average Normal Force by Contact Angle Bins During Plowing 
The normal displacement acting along the direction of the contact is determined by:

$$
\delta_{I}^{n}=\left(\delta^{r} \cdot n\right) n_{I}
$$

and the incremental shear displacement is the difference between the relative and normal displacement:

$$
\delta_{I}^{s}=\delta_{I}^{r}-\delta_{I}^{n}
$$

The updated normal and shear contact forces for the current time, $t$, are

$$
f_{I}^{n(t)}=f_{I}^{n(1-1)}+k_{n} \delta_{I}^{n}
$$

and

$$
f_{I}^{s(t)}=f_{I}^{s(t-1)}+K_{s} \delta_{I}^{s}
$$

A dry granular material does not allow tension forces, so the normal force must be checked for tension. If the normal force is found to be in tension, both the normal and shear forces for the contact are set to zero. If the normal force is found to be in compression, the shear force is evaluated against the friction limit:

$$
f^{s} \leq f^{n} \tan \phi
$$

If the shear force is set equal to the friction limit if shear force is greater than the frictional limit.

\subsection{Evaluation of the Smoothed Model}

Displacement data obtained from sampling the DEM results at the four material points were used to derive the smoothed system to compare the predicted stress of the smoothed model with measured stress from the DEM simulation.

Figure 5.16 compares the probability of contact angles of the DEM simulation and the data of the smoothed particle model, using 180 spokes, after $5 \mathrm{~mm}$ of plowing. Clearly, the smoothed model does not maintain the same contact angle distribution as the real granular material. This is because the smoothed model is only modeling the average displacements (i.e. modeling the granular material as an affine motion system). 


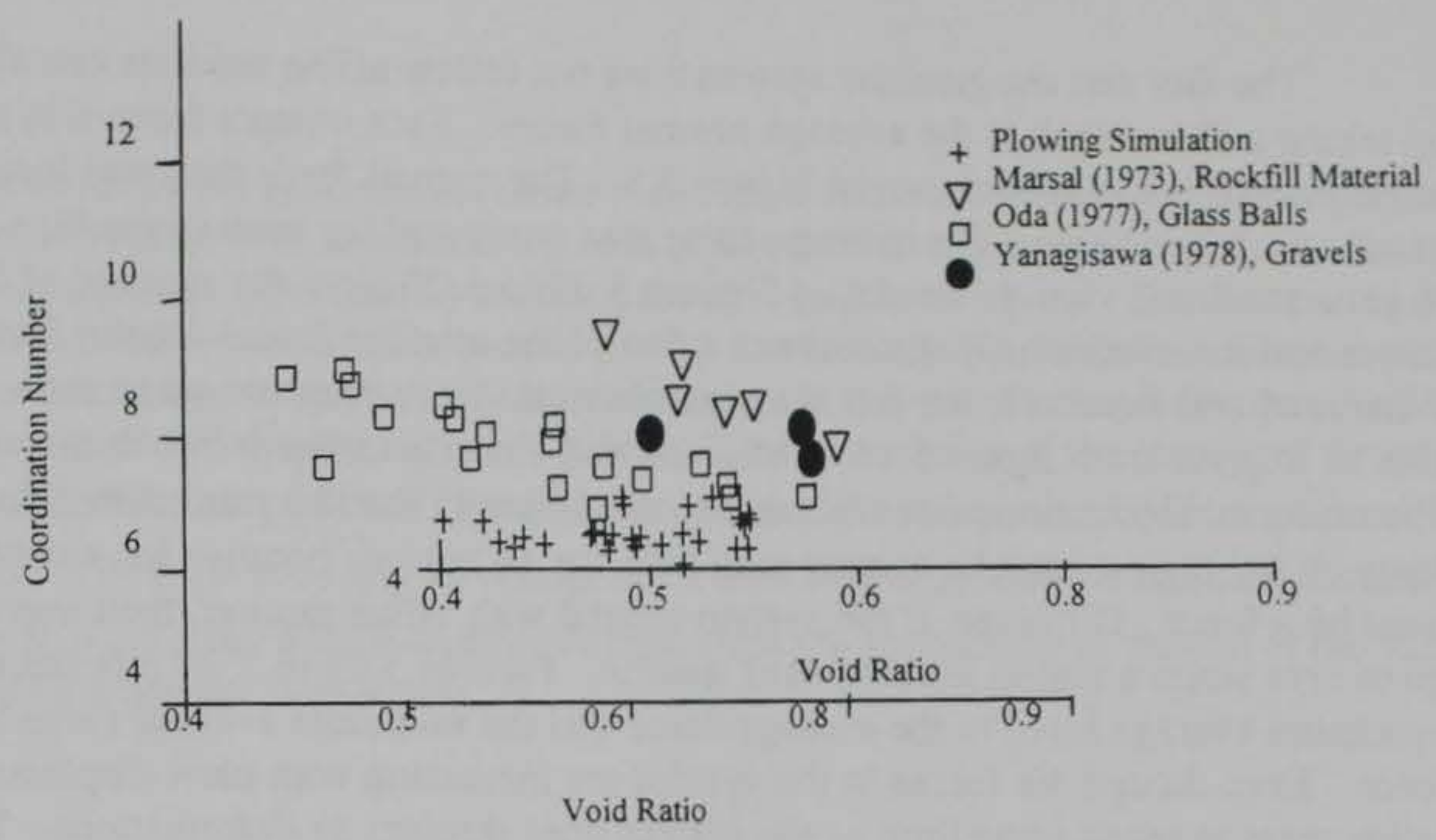

Figure 5.12: Coordination Number Vs. Void Ratio from Chang, 1989

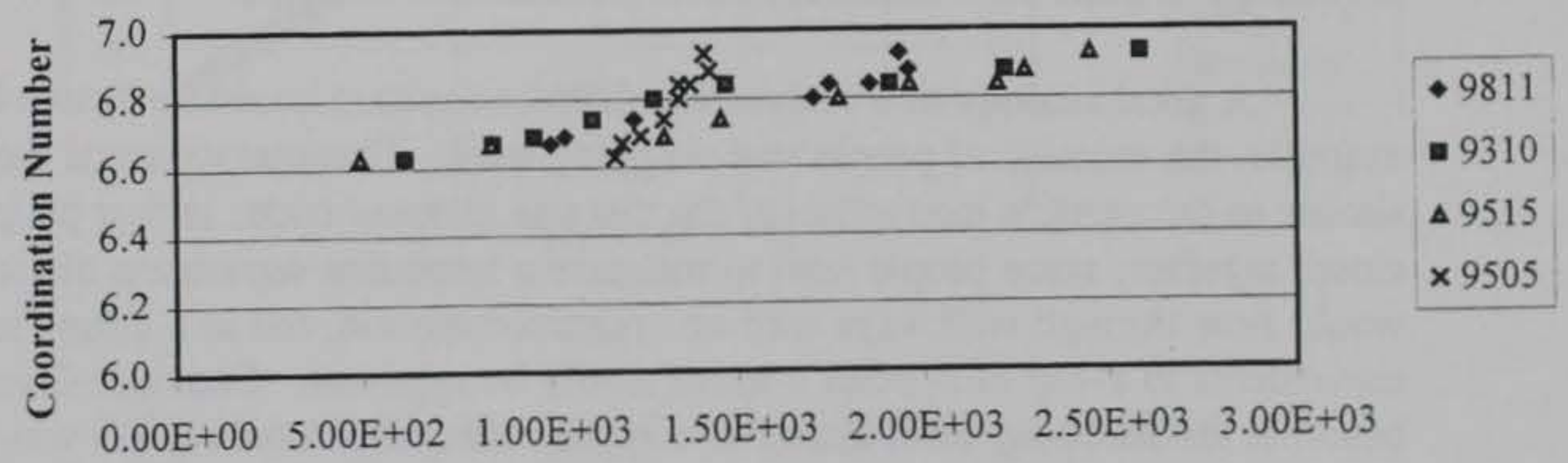

Mean Stress, Pascals

Figure 5.13: Relationship of Coordination Number and Mean Stress 
The fact that the granular system does not follow affine motions can also be observed by taking a closer look at the average normal forces. Each contact force was sorted into contact angle increments as was shown in Figure 5.8. The normal force data was lumped into six bins based on contact angle. The average force was computed for each of the bins. This was done to get a statistical view of the data. Figures 5.17 to 5.20 plots the average of the six average forces and the maximum and minimum value of the average forces for the four material points. What is of real interest is the fact that the minimum force does not go to zero. This fact could also be implied from figure 5.14, which showed a nearly uniform distribution of contact angles. The uniform distribution of contact angles would imply that the minimum values of average force in any region must be greater than zero for all regions because for a contact to occur there must be a force. However, if the system moved with affine motion, then average forces could go to zero when a region lends toward tension. Figures 5.21 to 5.24 plot the ratio of the maximum average force to the average force and the minimum average force to the average force. Even though the forces in the system are increasing with plow displacement, these two ratios seem to reach some limit as the system goes through its deformations. This could imply that the contact forces are being redistributed uniformly and proportionately in angle space as the granular system is deformed.

From the previous observations, it is apparent that there should be some mechanism that accounts for the forces and contact angles generated from the non-affine motions. One way of modeling the effect of the non-affine motions would be to treat this system as a convectiondiffusion system in which the physical properties being modeled are obtained from breaking the model into two parts based on average motion, plus a component that is a result of the deviation from the average motions. This type of approach is used to model many other systems, such as the change in pollution concentration in groundwater flow [5].

A good analogy of a convection-diffusion system could be drawn by using, as an example, the velocity of people in a shopping mall. The interaction of the people in the mall is similar to the particle interaction of the discrete element code, in that people avoid coming closer together, since people tend to maintain a minimum separation distance. The crowd would flow through walkways with an organized motion; but at a closer look, the random movements to avoid each other's space would be apparent. Consider if you were sitting on a bench in the shopping mall, acting as a spatial observation point, and you had a way of instantaneously sampling the velocity of each person passing you in the region. You could develop a spatial velocity gradient for your immediate area. If you wished to predict the location of a person over time, you could apply the average velocity obtained from your sample and integrate over time to estimate the location of the individual. Obviously, after a length of time, the accuracy of the estimate would degrade because no one is really travelling at the average velocity. The movements to avoid each person's space would create deviations from the average motion. Likewise, in the particle system, the use of only the smoothed velocity of the system will provide an increasingly poorer estimate of the current condition of the system with time. 
The following presentation of the convection-diffusion system was developed from a presentation by Bear [5]. In general the convection-diffusion system describes the change in the value of a parameter of interest by dividing the change into two parts. The first part is described by an average motion, $\mathrm{A}$, and the second part by a uniformly distributed random component, $\mathrm{B}$. For a given time step the probability that the change in the quantity is greater than $\mathrm{A}+\mathrm{B}$ or less than $\mathrm{A}-\mathrm{B}$ is zero. Within the region $\mathrm{A}-\mathrm{B}$ to $\mathrm{A}+\mathrm{B}$ the probability of the change of the quantity being $x$ is defined as $p(x)=B / 2$. Because the random component is distributed uniformly around $A$. The expected value of change for a time step is $A$ and the standard deviation would be $\sigma^{2}=B^{2} / 3$. After a large number of time steps the probability of change of the value can be described by a normal distribution:

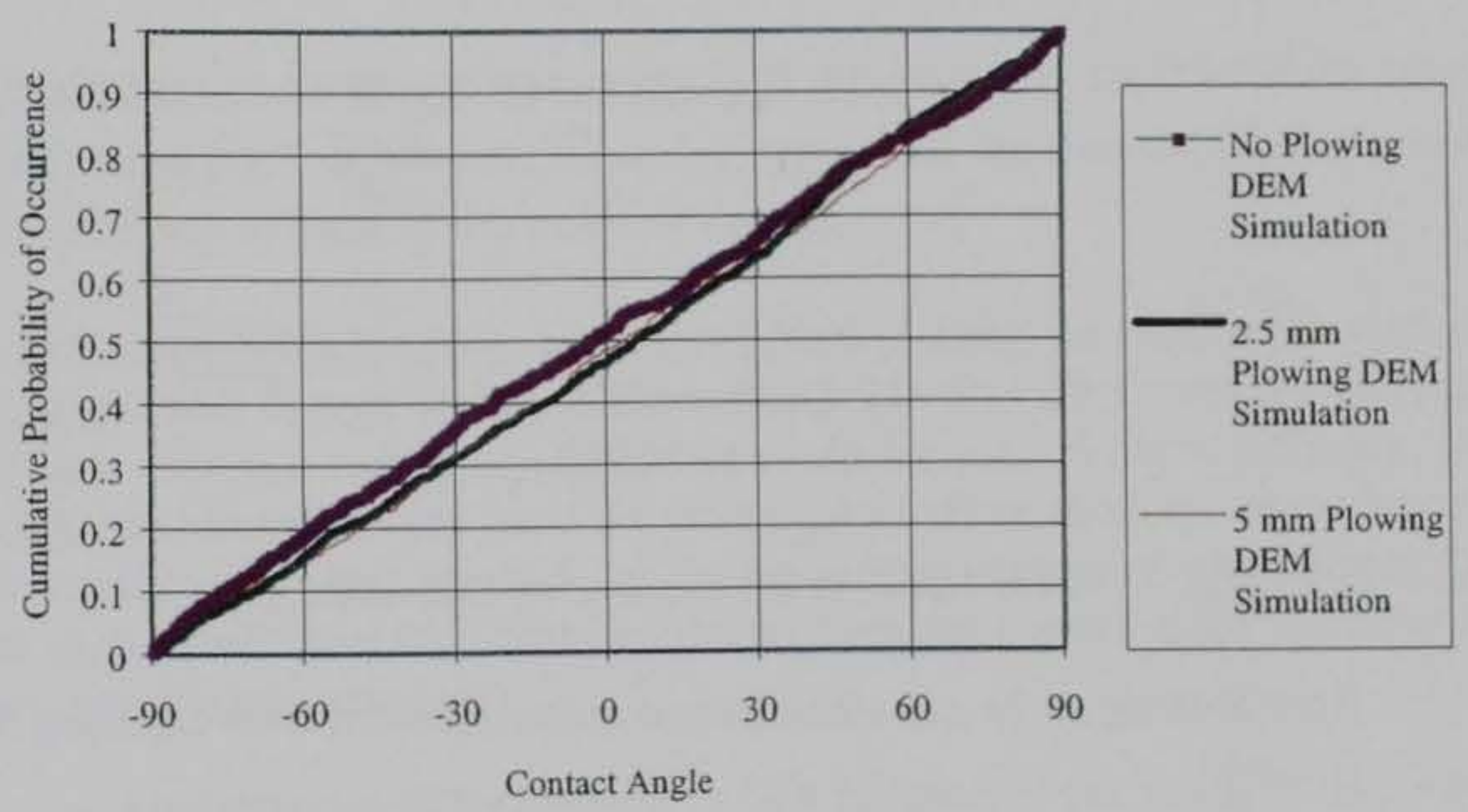

Figure 5.14: Distribution of Contact Angles During Plowing 

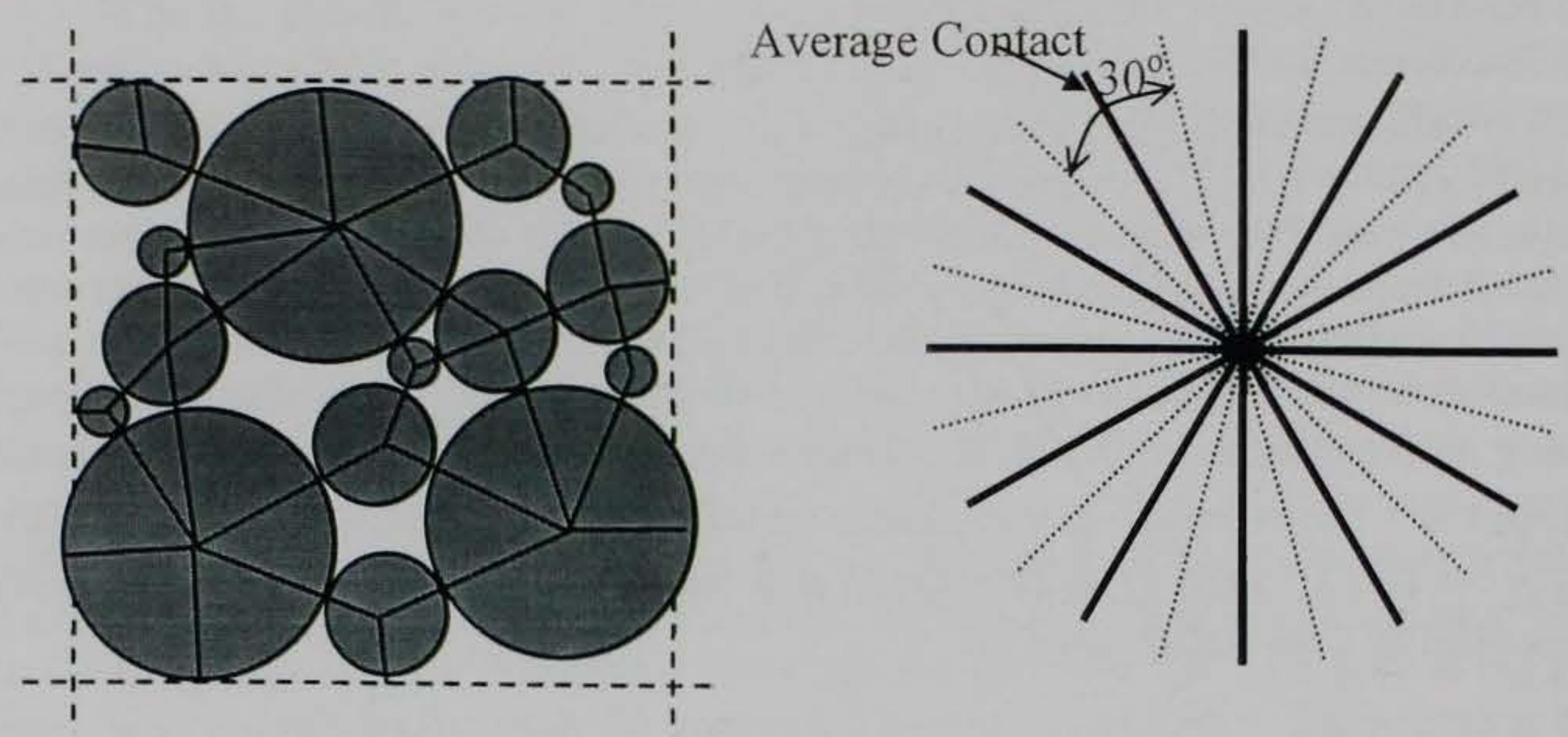

Figure 5.15: Conceptual Design of Spoke Model

$$
p(x)=\frac{1}{\sqrt{2 \pi} S^{2}} \exp ^{-\frac{(x-E V)^{2}}{2 s^{2}}}
$$

where $E V=T A, S^{2}=T B^{2} / 3, T$ is the number of time steps.

Now looking at the one-dimensional convection-diffusion equation that is described as:

$$
\frac{\partial c}{\partial t}=V \frac{\partial c}{\partial x}+\frac{\partial^{2} c}{\partial x^{2}}
$$

where $-V \frac{\partial c}{\partial x}$ is the change associated with the average flow of the system, $D \frac{\partial^{2} c}{\partial x^{2}}$ is the change associated with the random component of the system.

The solution to Equation (5.18) is

$$
\frac{c}{c_{o}}=\frac{1}{\sqrt{4 \pi D t}} \exp ^{-\frac{(x-V t)^{2}}{4 D t}}
$$


A comparison of Equation (5.19) and (5.17) shows that both solutions are of the same form and that

$$
\begin{aligned}
& E V=V t \\
& S^{2}=2 D t
\end{aligned}
$$

To compute the value of the quantity that results from the diffusion process, a onedimensional discrete version of Equation (5.18) can is used:

$$
\Delta c=\Delta t D \frac{c^{-}-2 c^{0}+c^{+}}{\Delta x^{2}}
$$

where $\Delta C$ the change in the property as a result of diffusion. $c^{0}$ represents the smooth value of the property at the point of interest. $c^{-}$and $c^{+}$represent the smooth value property at points just in front of and just in back of the point of interest.

The diffusion coefficient, $D$, is obtained from plotting the standard deviation of the quantity of interest with time as shown in Equation (5.21). Particle forces are carried on contacts that have certain a certain orientation or contact angle. Without diffusion, the change in contact angles would result only from the smoothed or affine motions. In order to capture the change in contact angle that results from the non-affine motions, the deviation of the true contact angle as sampled from the DEM simulation from the contact angle resulting from smoothed deformation was measured.

Because the deviations in forces tend to be a function of the strain of the system, the time variable in Equation (5.21) must contain more than just a temporal measurement of the system. This situation is similar to the concept of intrinsic time has been used in endochronic theory to better describe the state of system [51]. Intrinsic time is defined as a monotonically increasing scalar of strain and time. The time intergrated magnitude of the rate of deformation tensor was used at the intrinsic time variable for this system. Figure 5.25 show the relationship between the standard deviation of the average contact angle and the time intergrated magnitude of the rate of deformation tensor, $D_{i j}$. In general, the deviation of the contact angle increases with an increase of the magnitude of the rate of deformation tensor. In view of Equation (5.21). This would imply that the diffusion model is a good analogy for describing the random component of forces of the granular system. The slope of a line passing through the data points in Figure (5.25) represents the diffusion coefficient for the system. 


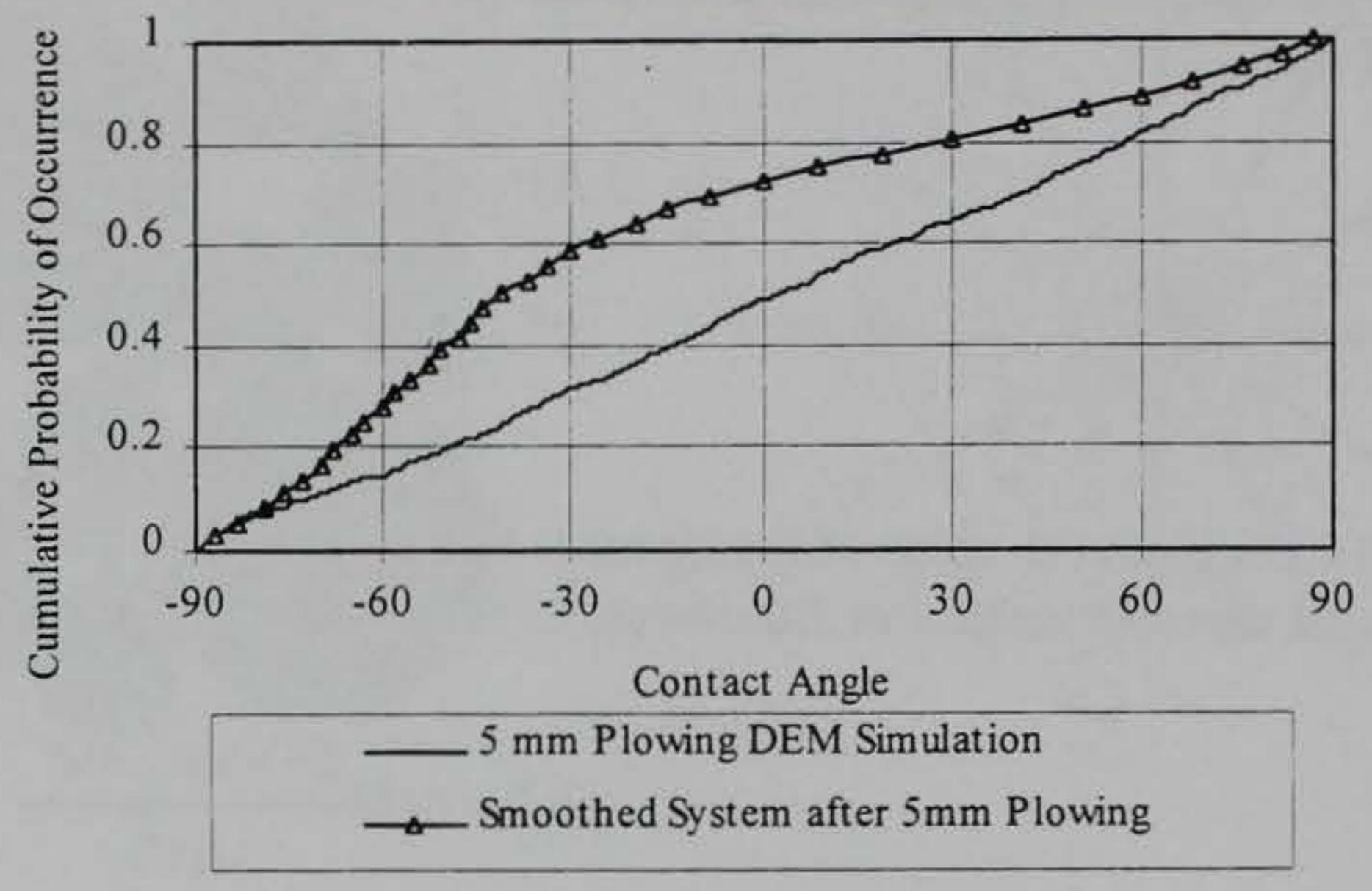

Figure 5.16: Distribution of Contact Angles from Smoothed Spoke System after $5 \mathrm{~mm}$ of Plowing

By adding the diffusion to the smoothed system, the smoothed system shows more realistic behavior. In Figure 5.26, the ratio of the maximum average force to the average force and the minimum average force to the average force for the DEM simulation, plots smoothed system without diffusion and smoothed system with diffusion. Clearly the addition of the diffusion component has made the force ratio of the smoothed system behave more like that of the DEM simulation. Figure 5.27 compares the cumulative probability of occurrence of contact angle of the 12 spoke smoothed system with diffusion, a 180 spoke smoothed system without diffusion, and the DEM simulation. From this plot, it is observed that the smoothed system now maintains a contact angle distribution similar to that of the real granular system.

Figure (5.28) to (5.31) are the results from the DEM simulation and the smoothed system for shear stress to shear strain for the four sampling points. The smoothed system has good agreement with the results of the DEM simulation. For both points 9811 and 9310 , the smoothed system produces a yield stress that is similar to the DEM simulation. For point 9515 which is a point at which yielding has not occurred, the smoothed system matches the increase of shear stress with shear strain. Even the smoothed system has good agreement at point 9505 with the DEM simulation even though this point has been subjected to only small strain.

Figure (5.32) to (5.39) compare the p-q plots of the DEM simulation and the smoothed system simulation. $\mathrm{p}$ is defined as:

$$
p=\frac{\sigma_{1}+\sigma_{3}}{2}
$$


and

$$
q=\frac{\sigma_{1}-\sigma_{3}}{2}
$$

It is expected that the worst fit when comparing the DEM to the smoothed system would be in the p-q plots because the simulations are strain driven. Therefore, the quality of the model predictions is based on the ability to replicate stress. The shape of the plot for point 9811 is predicted although there are differences in detail. For point 9310 , both DEM and the smoothed system drift up to the correct $q$ in about the correct range of $p$. For point 9515 , the stresses are both over-predicted; although the ratio $q / p$ is correct. Hence the good prediction of stress ratio. This is at a low stress range and error is probably magnified. Point 9505 has a good prediction, especially considering the small strain. This is a very different stress path, yet was predicted well. In general, it is doubtful that any constitutive model could have done as well. The results are especially amazing considering that the parameters come from micro-measurements.

Figures 5.36 to 5.39 compare the development of the stress ratio for the DEM simulation and the Smoothed system simulation. The stress ratio from the smoothed system compared very well with the measured results from the DEM simulation.

Based on these results, it appears that the smoothed model captures most of the significant microscopic statistics and macroscopic mechanical behavior obtained from the DEM simulation. The sampling points selected for evaluating the model represented a wide variant in imposed strain history, but in each case the smoothed system provided the correct trend. 


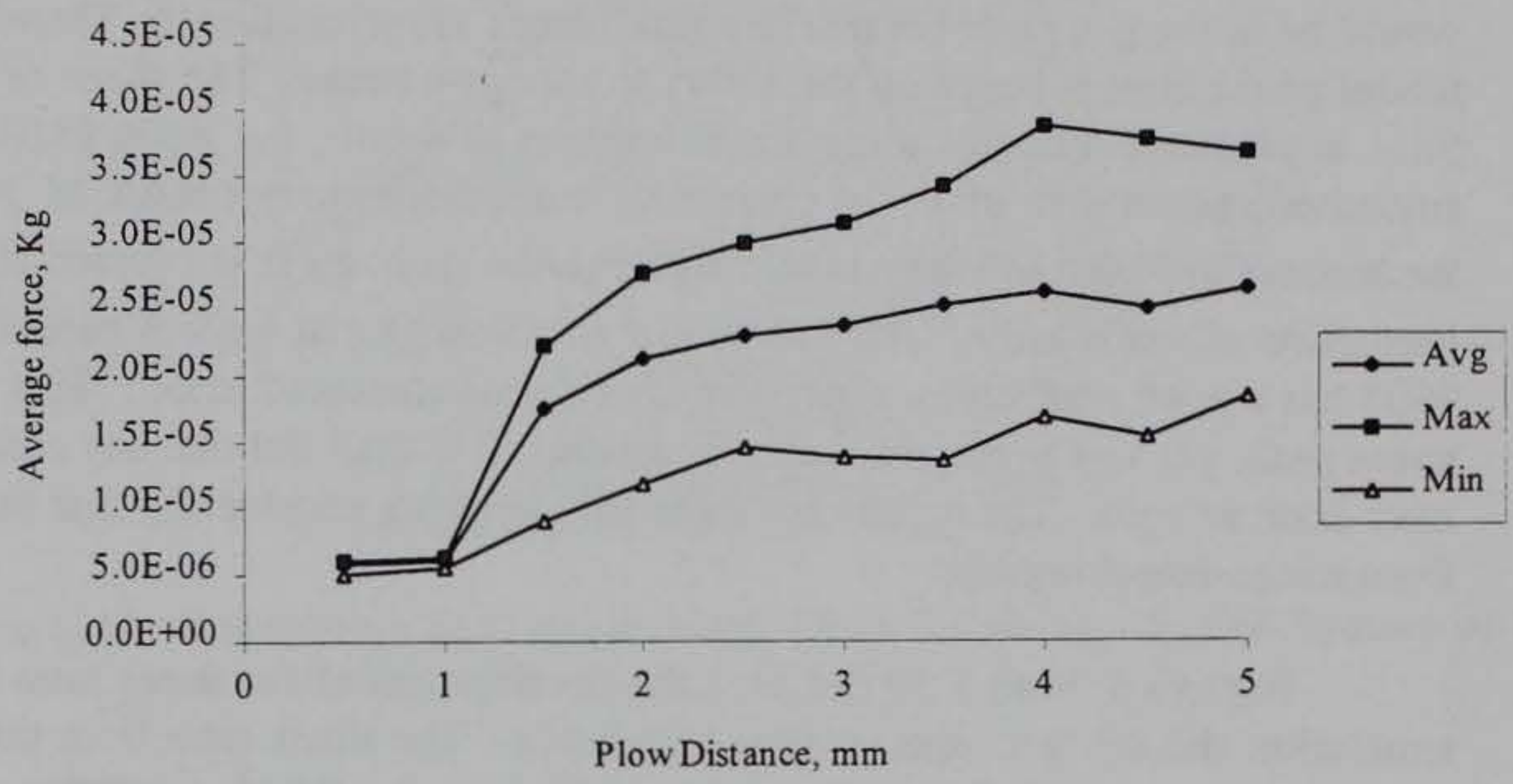

Figure 5.17: Average Normal Force for Material Point 9310

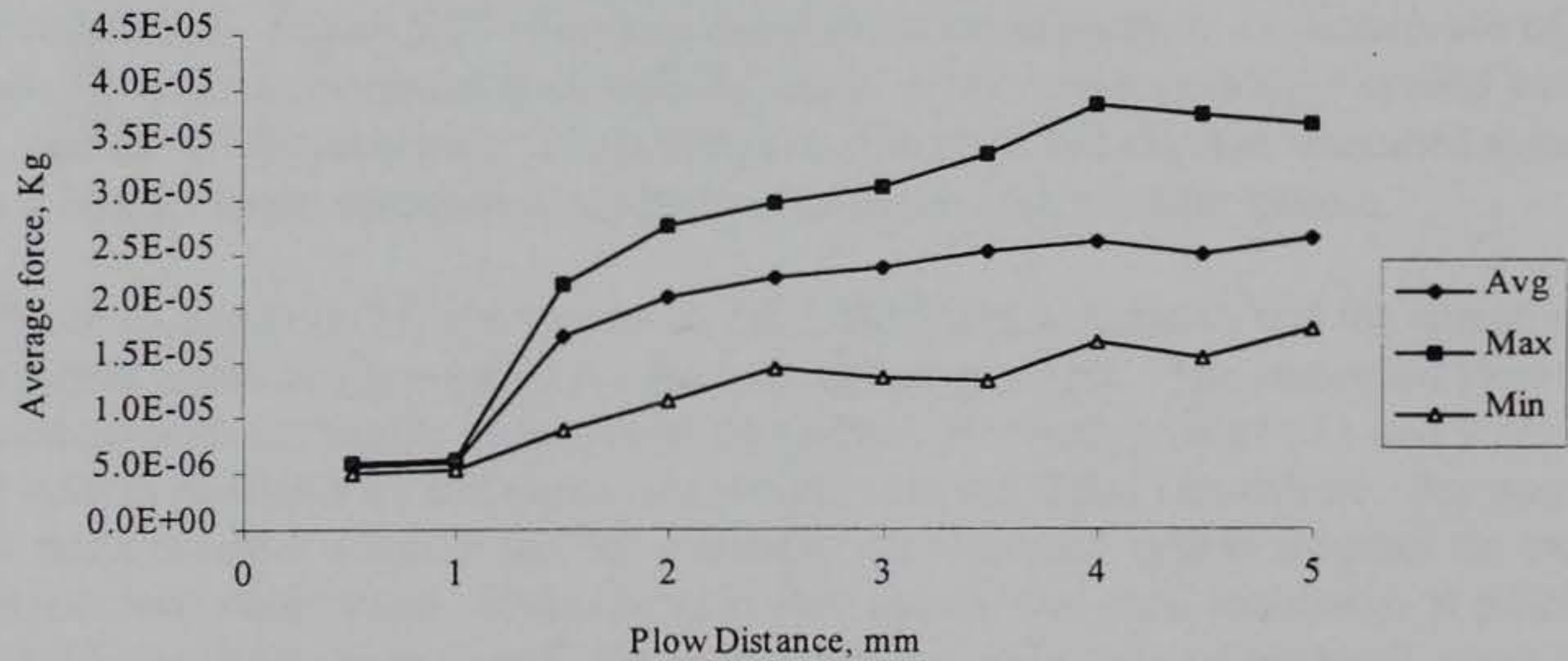

Figure 5.18: Average Normal Force for Material Point 9811 


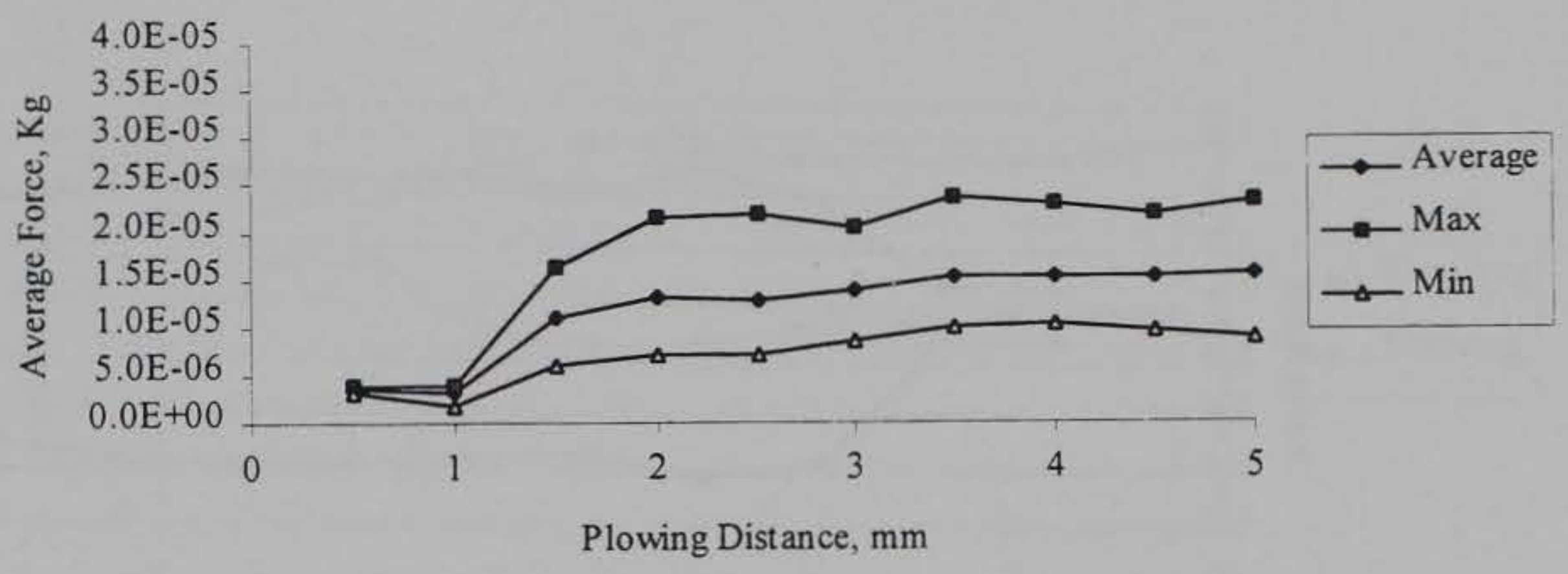

Figure 5.19: Limits on Average Normal Force for Material Point 9515

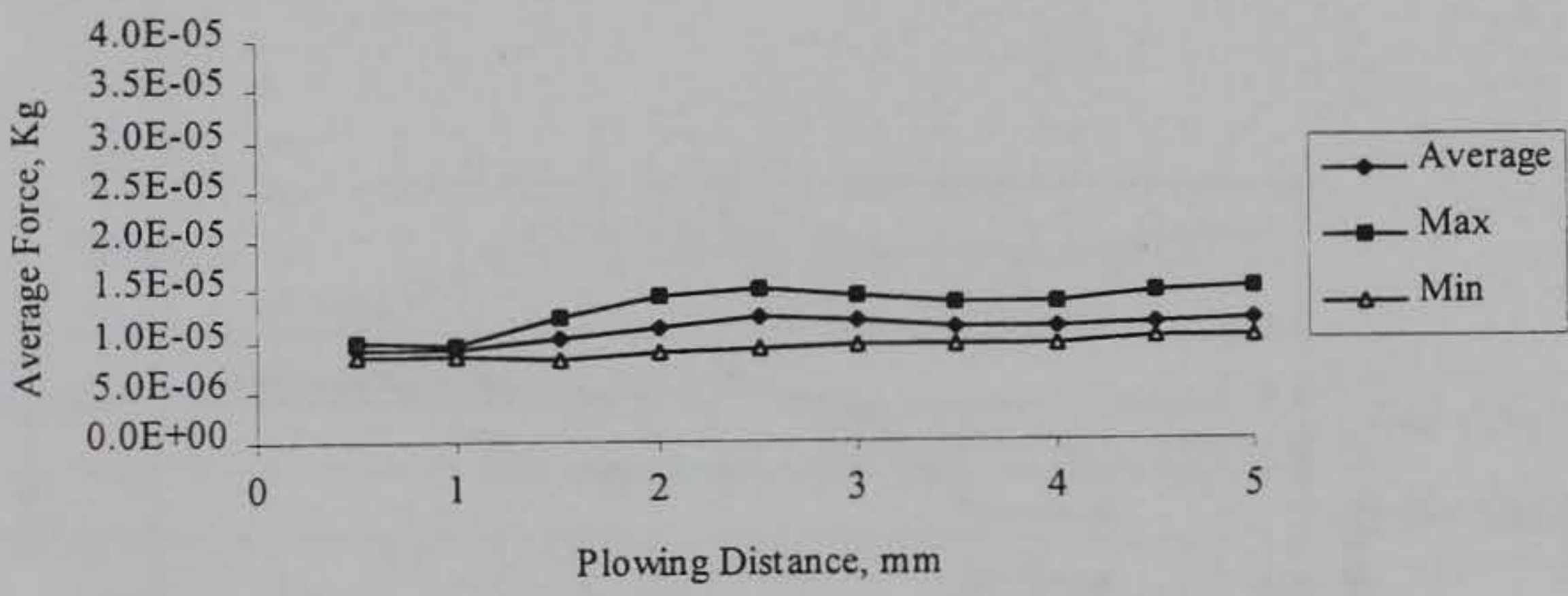

Figure 5.20: Average Normal Force for Material Point 9505 


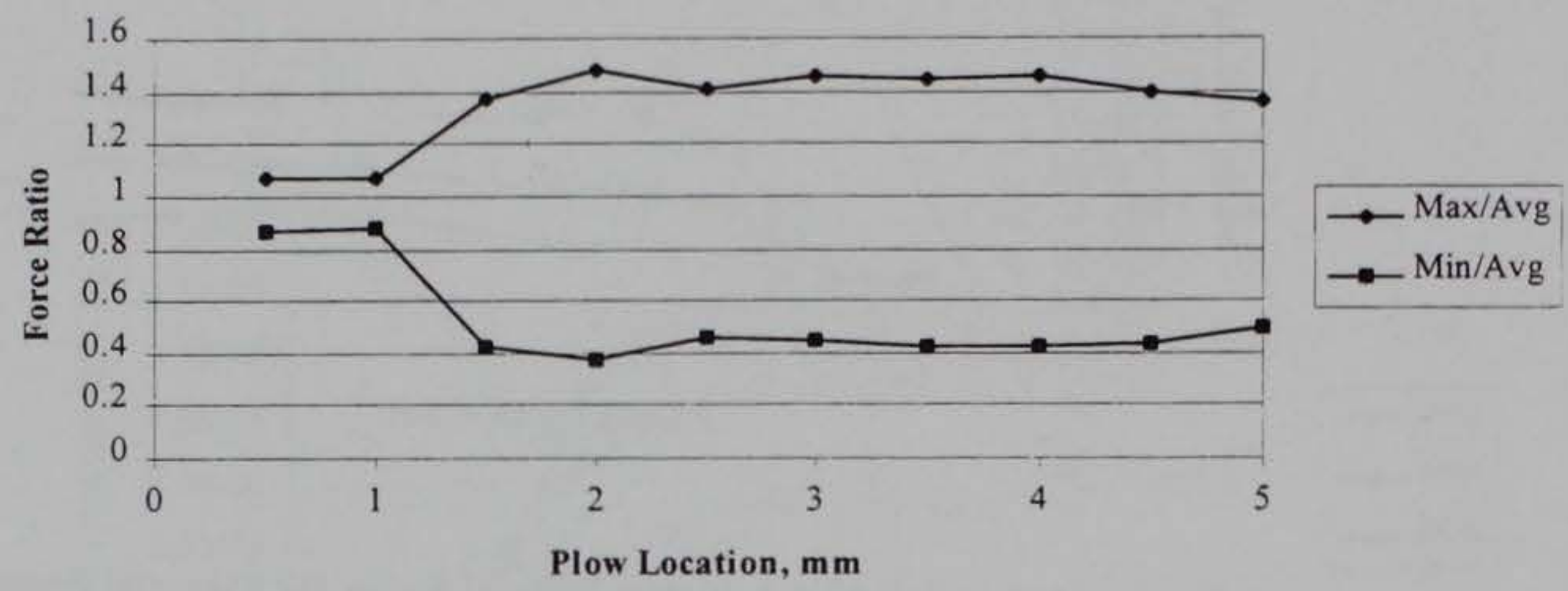

Figure 5.21: Limits on Average Normal Force for Material Point 9310

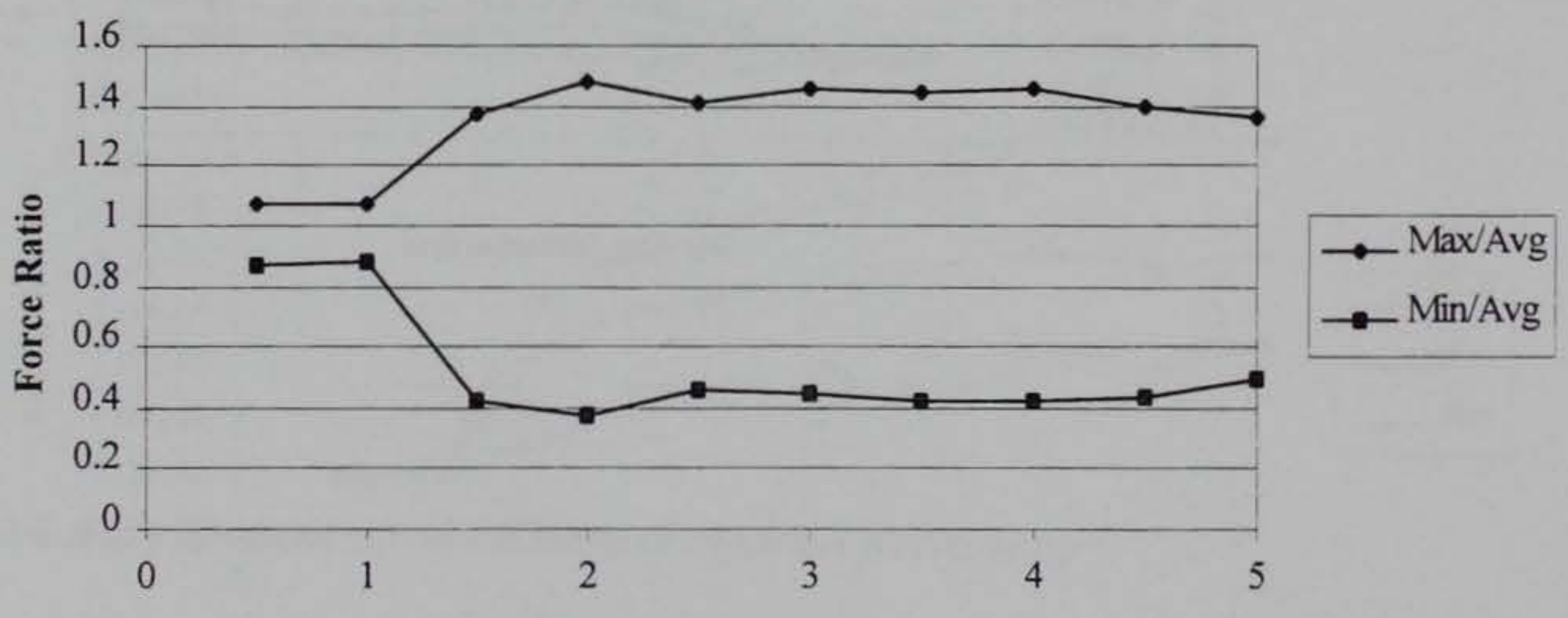

Plow Distance, $\mathrm{mm}$

Figure 5.22: Limits on Average Normal Force for Material Point 9811 


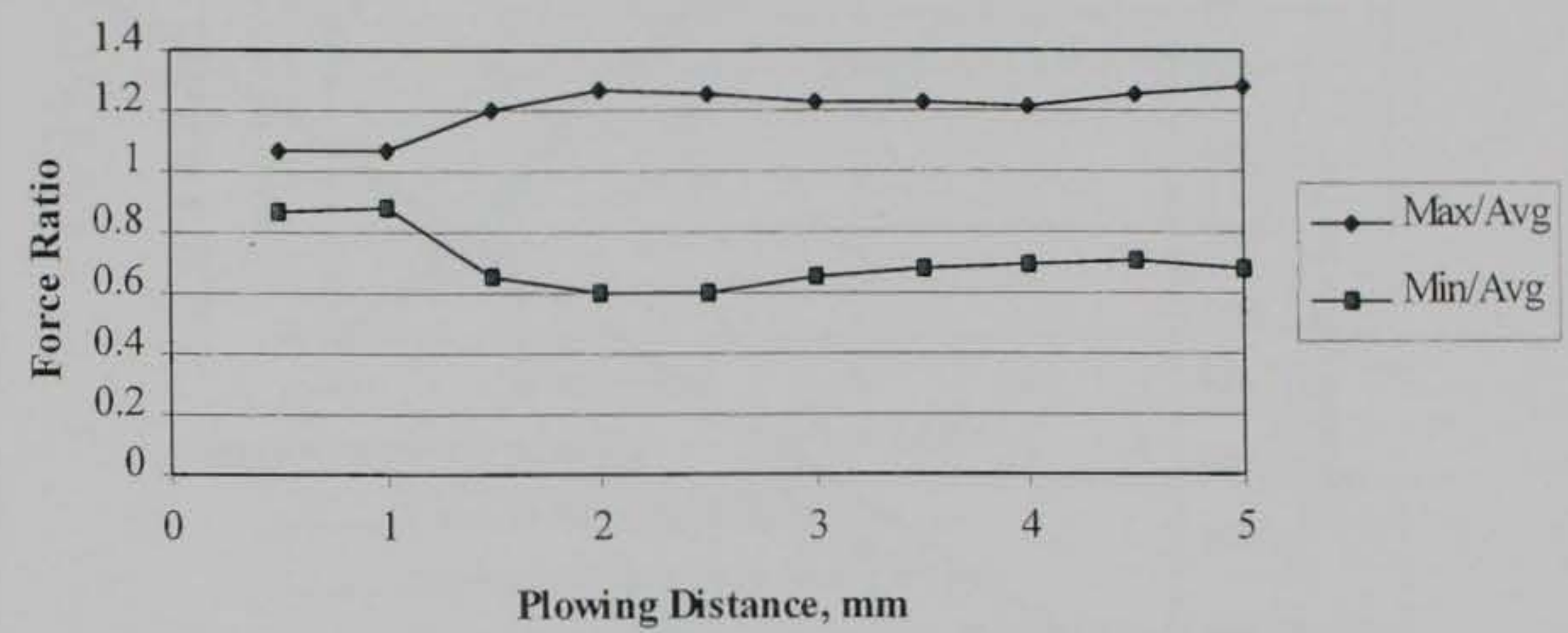

Figure 5.23: Limits on Average Normal Force for Material Point 9515

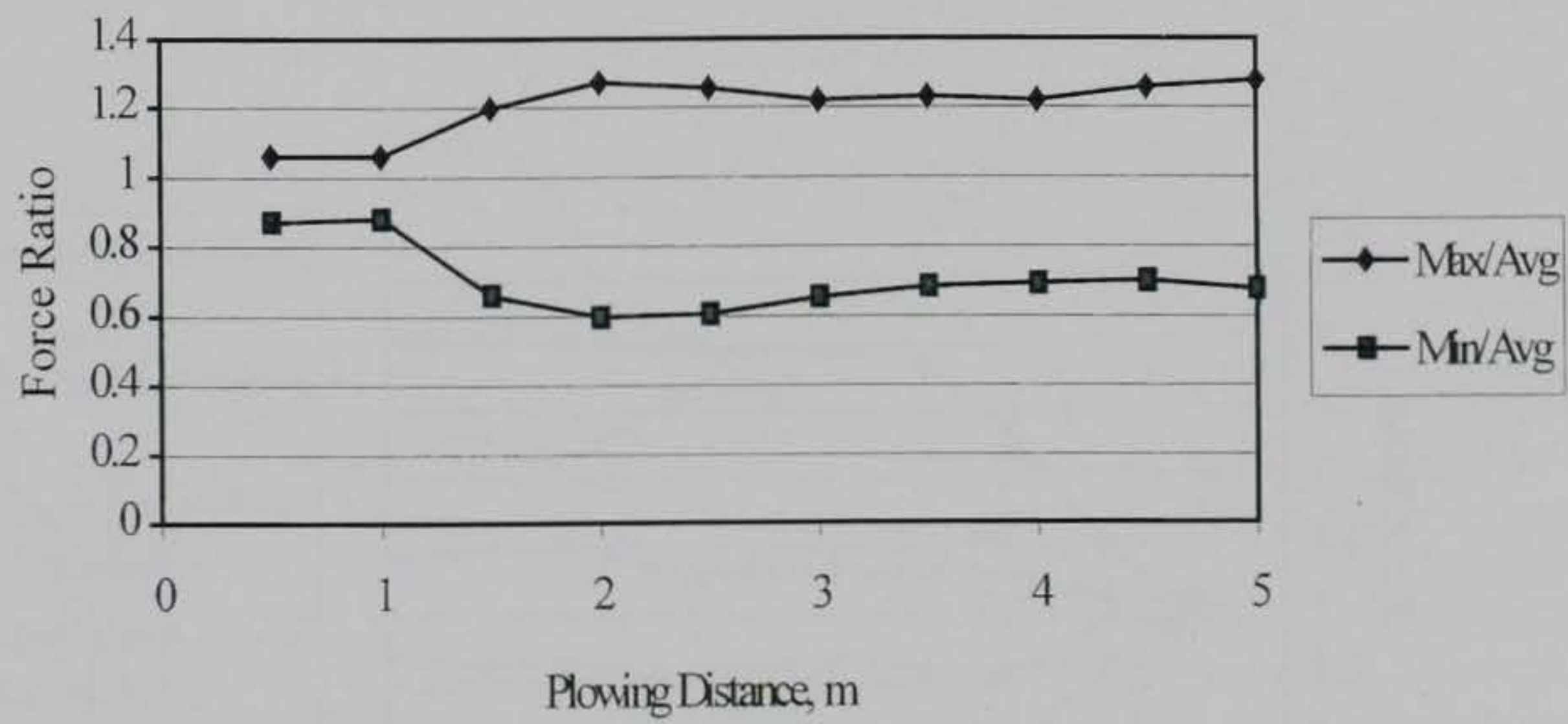

Figure 5.24: Limits on Average Normal Force for Material Point 9505 


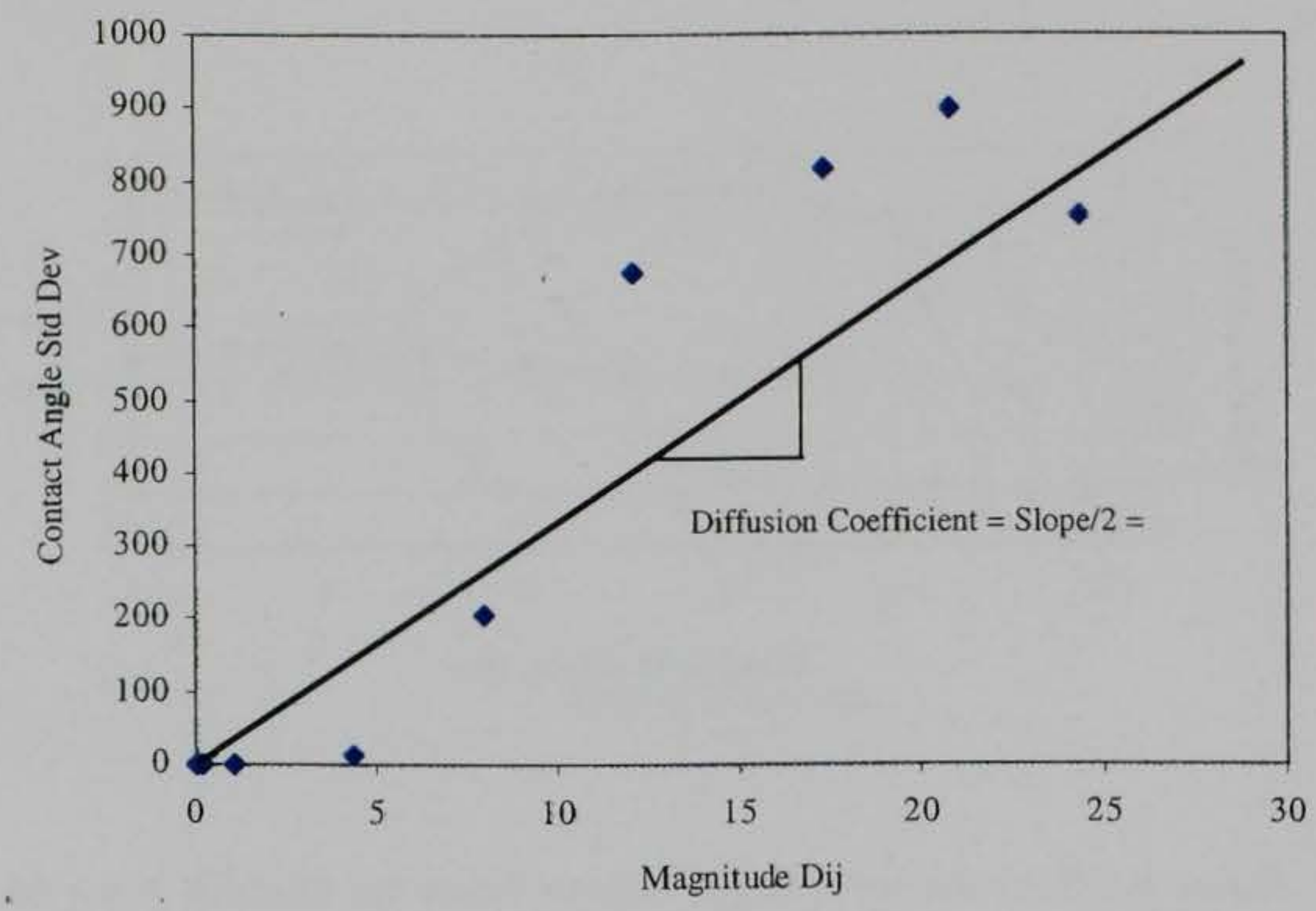

Figure 5.25: Contact Angle Diffusion
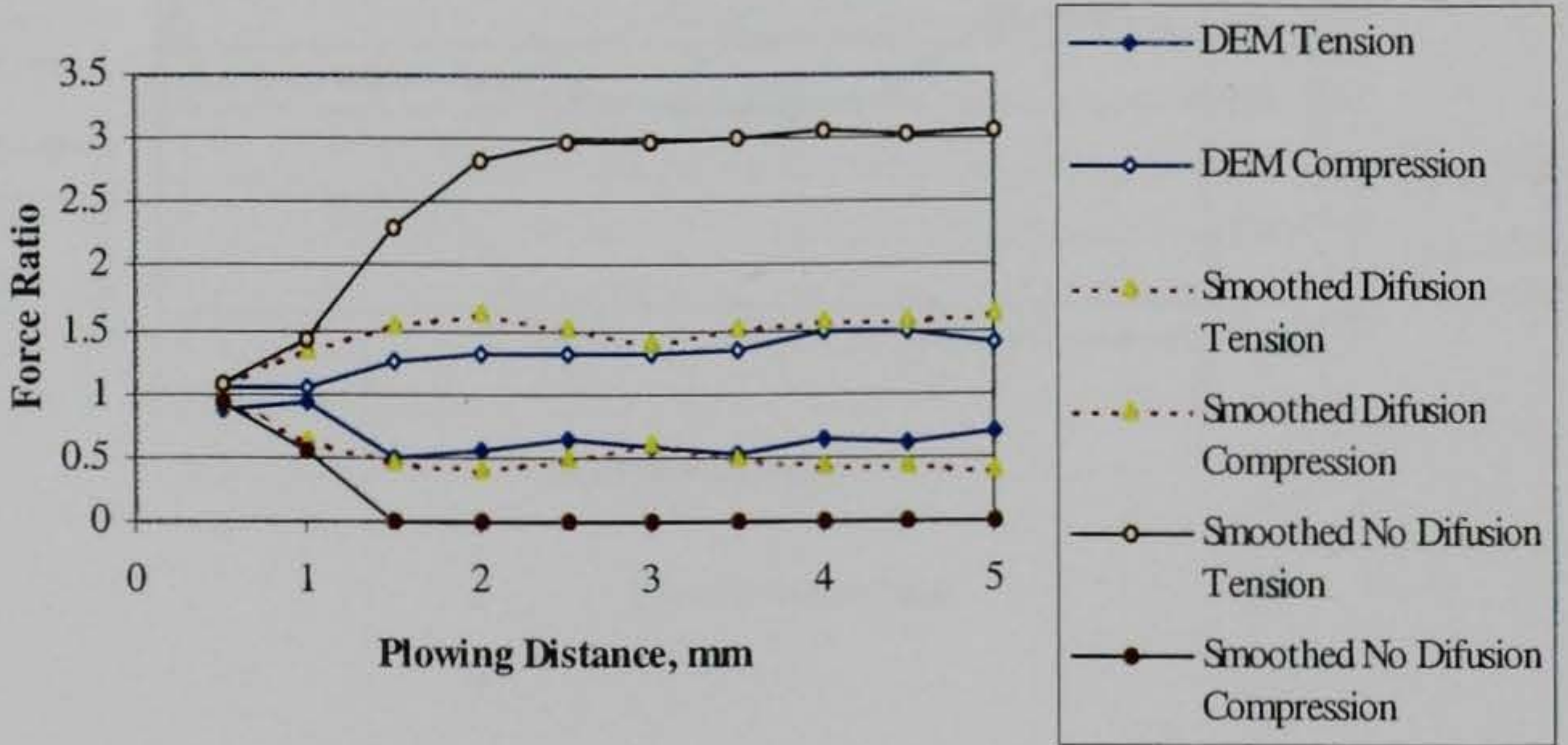

Figure 5.26: Comparison of Force Limits for DEM and Smoothed System, Point 9811 


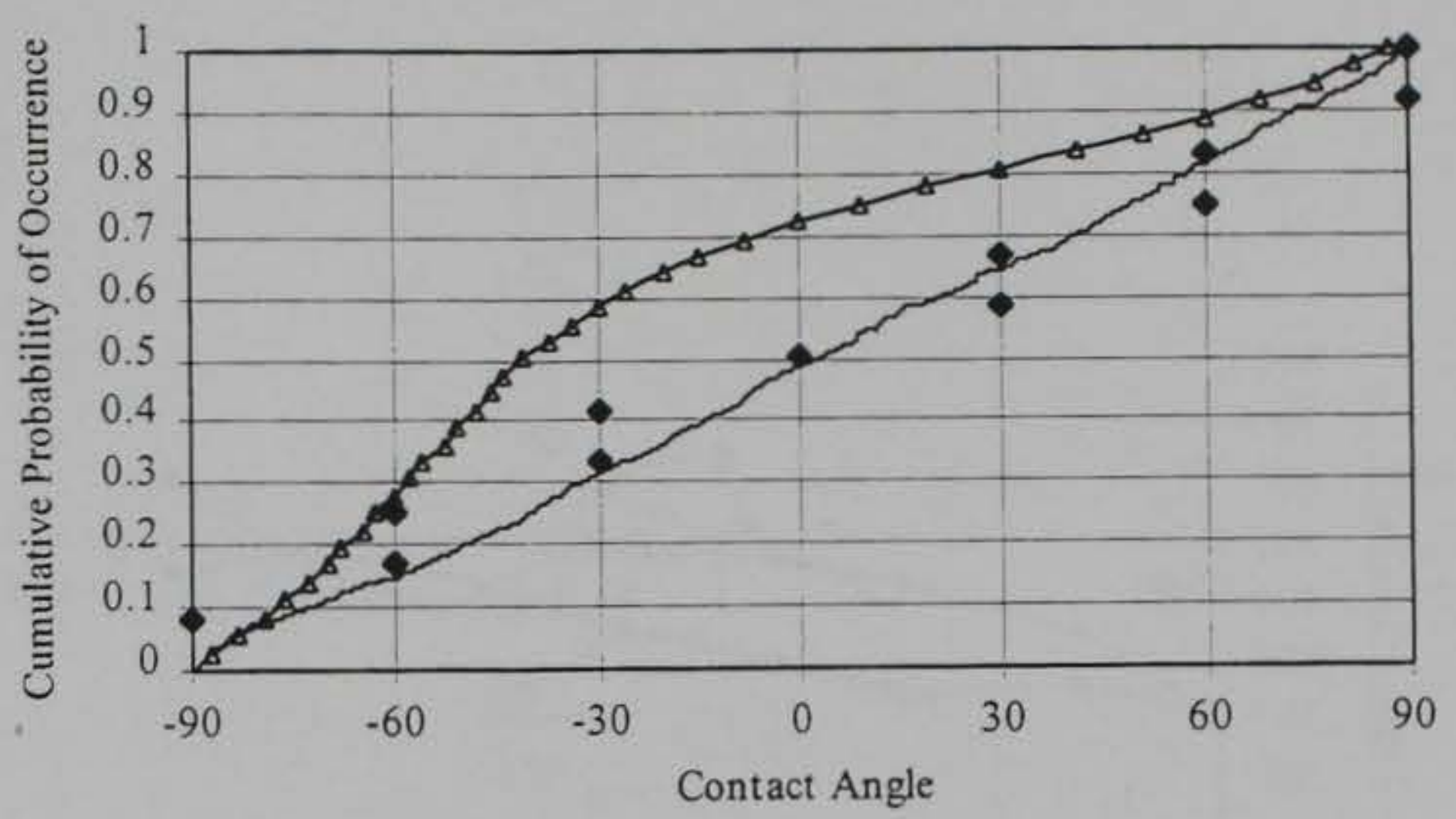

$5 \mathrm{~mm}$ Plowing DEM Simulation

$\longrightarrow$ Smoothed System after $5 \mathrm{~mm}$ Plowing

- 12 Contact Smoothed System with Difusion after $5 \mathrm{~mm}$ Plowing

Figure 5.27: Distribution of Contact Angles from Smooth System with Diffusion

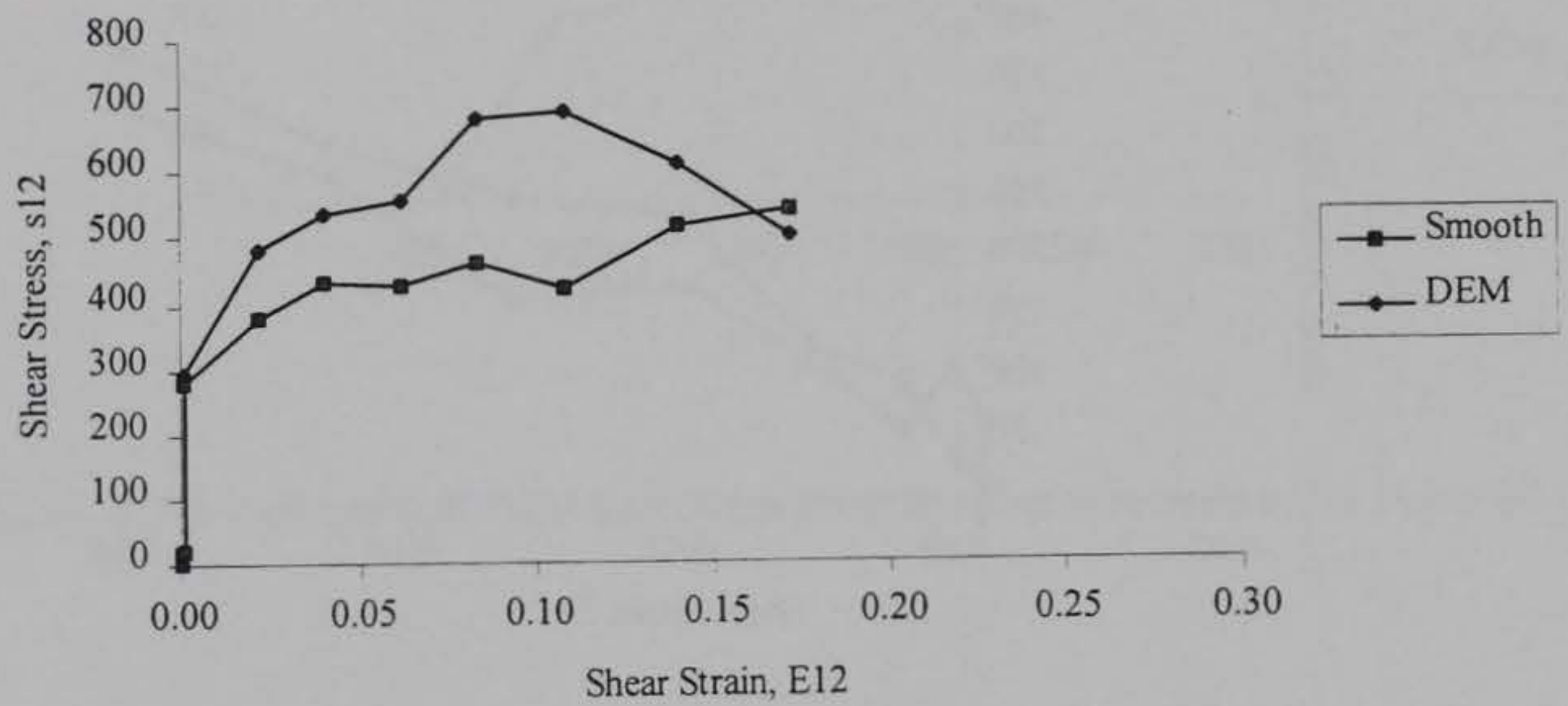

Figure 5.28: Comparison DEM and Smoothed System Shear Stress for Point 9310 


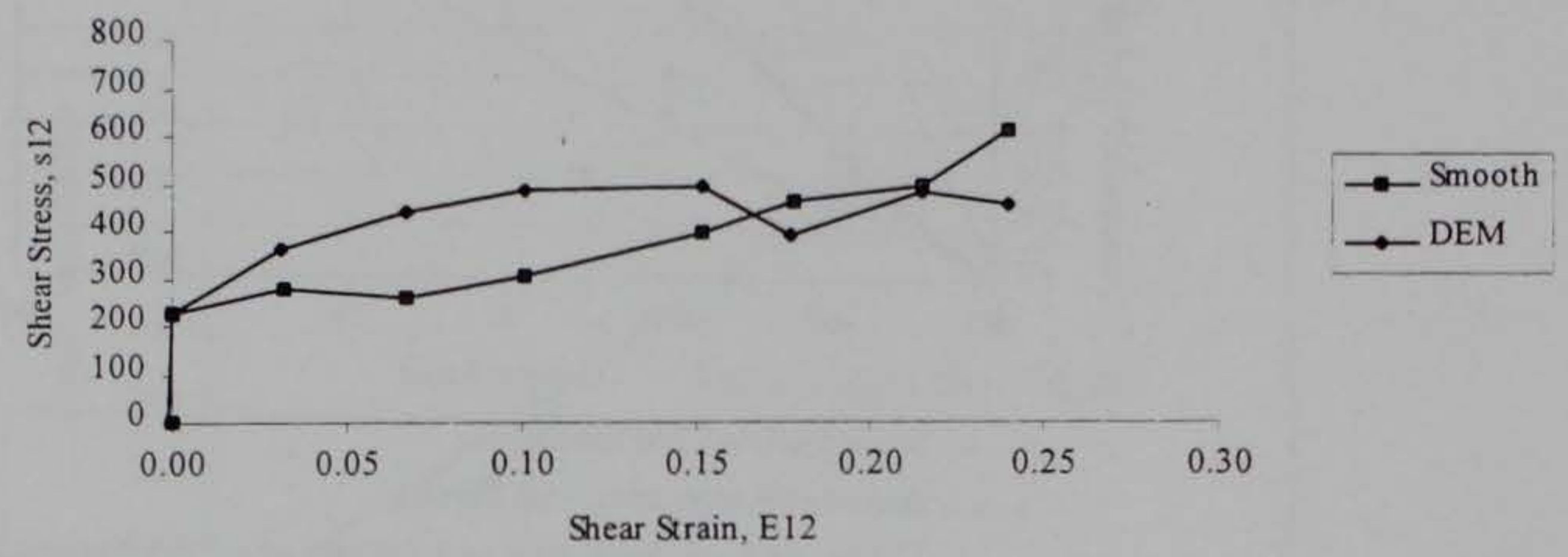

Figure 5.29: Comparison DEM and Smoothed System Shear Stress for Point 9811

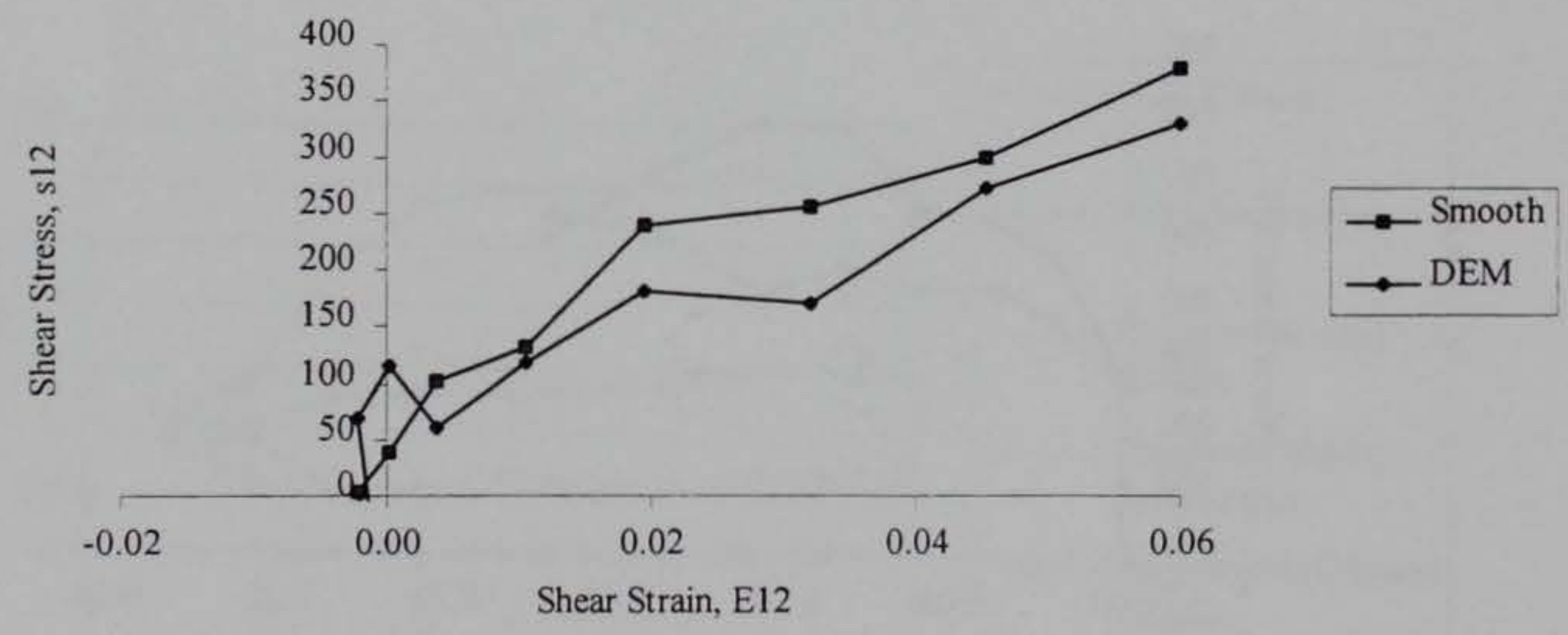

Figure 5.30: Comparison DEM and Smoothed System Shear Stress for Point 9515 


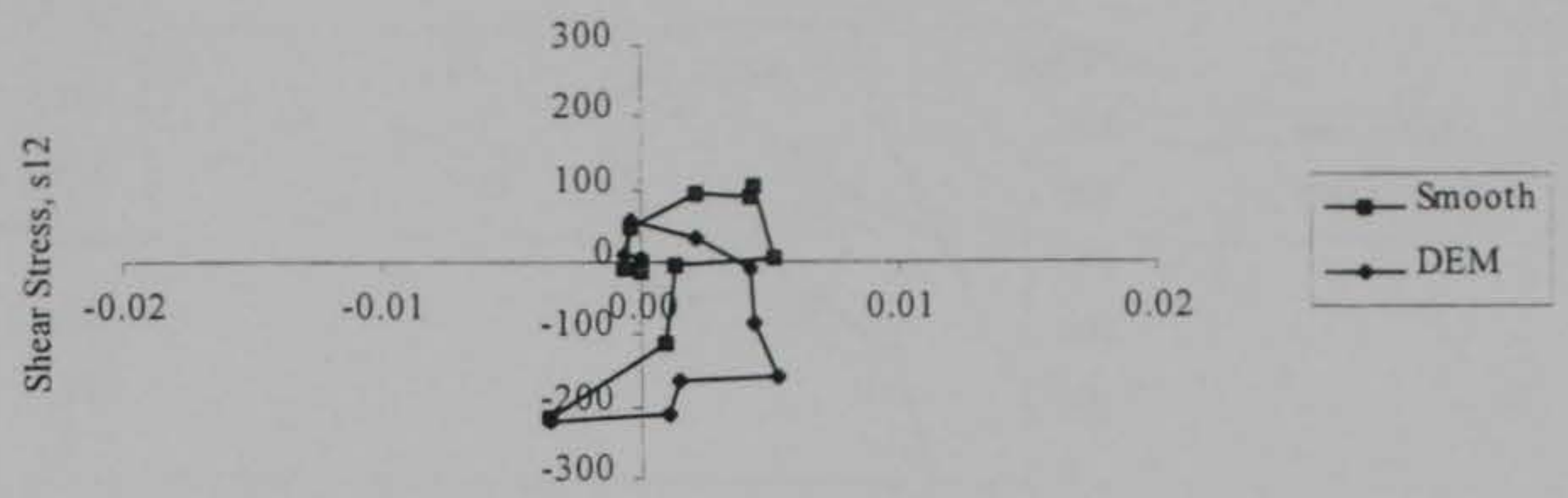

Shear Strain, E12

Figure 5.31: Comparison DEM and Smoothed System Shear Stress for Point 9505

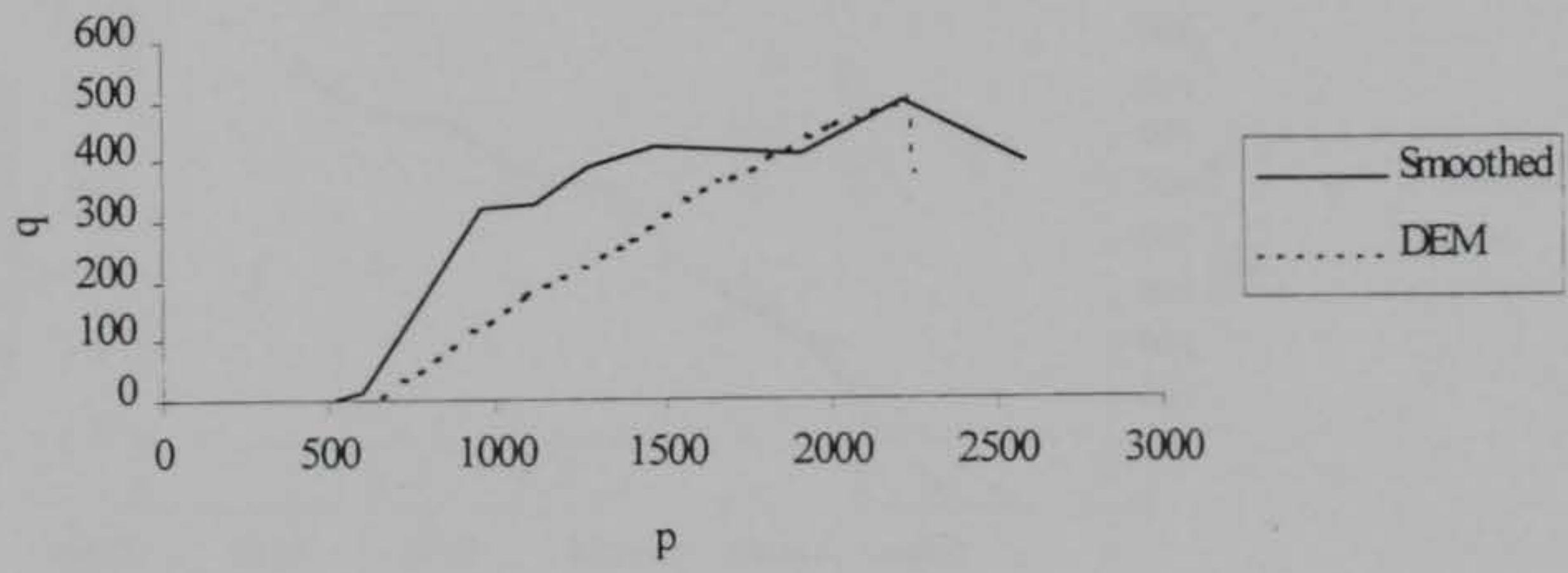

Figure 5.32: Comparison DEM and Smoothed System Stress Path for Point 9310 


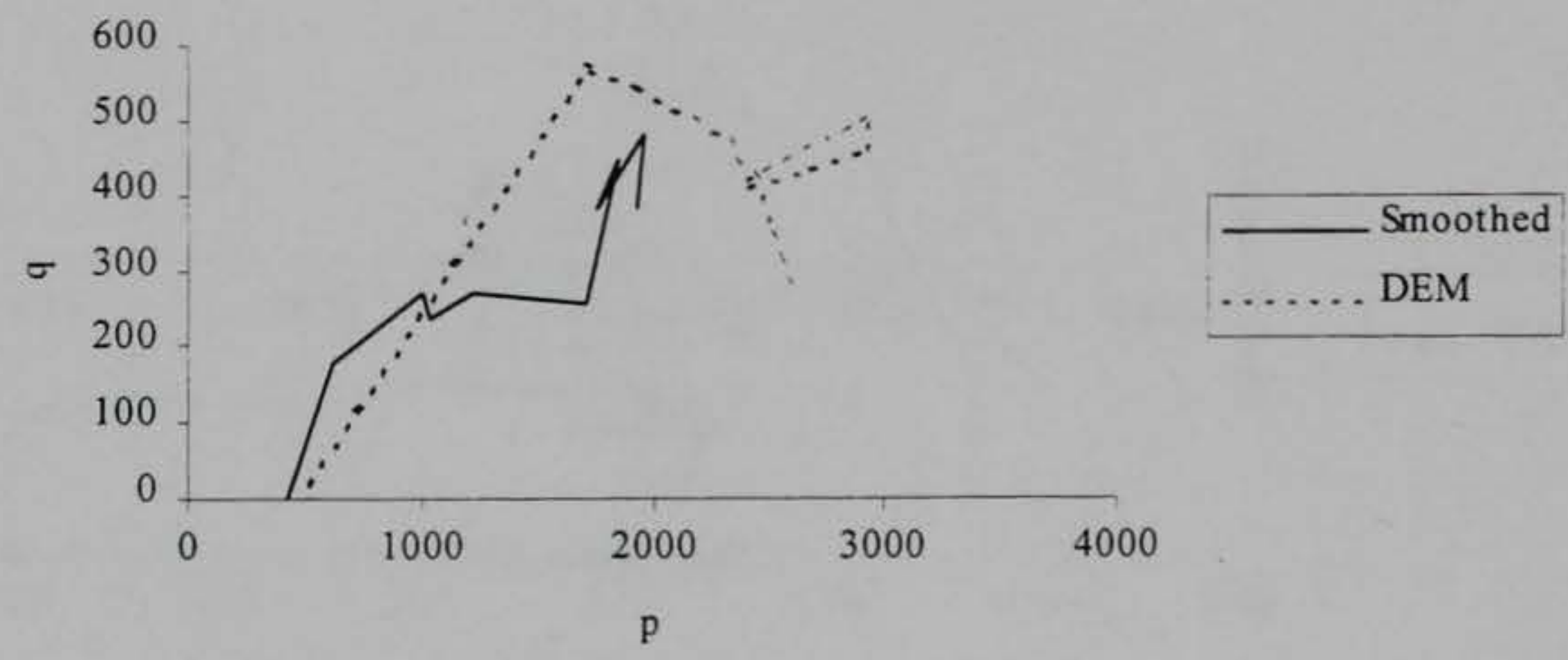

Figure 5.33: Comparison DEM and Smoothed System Stress Path for Point 9811

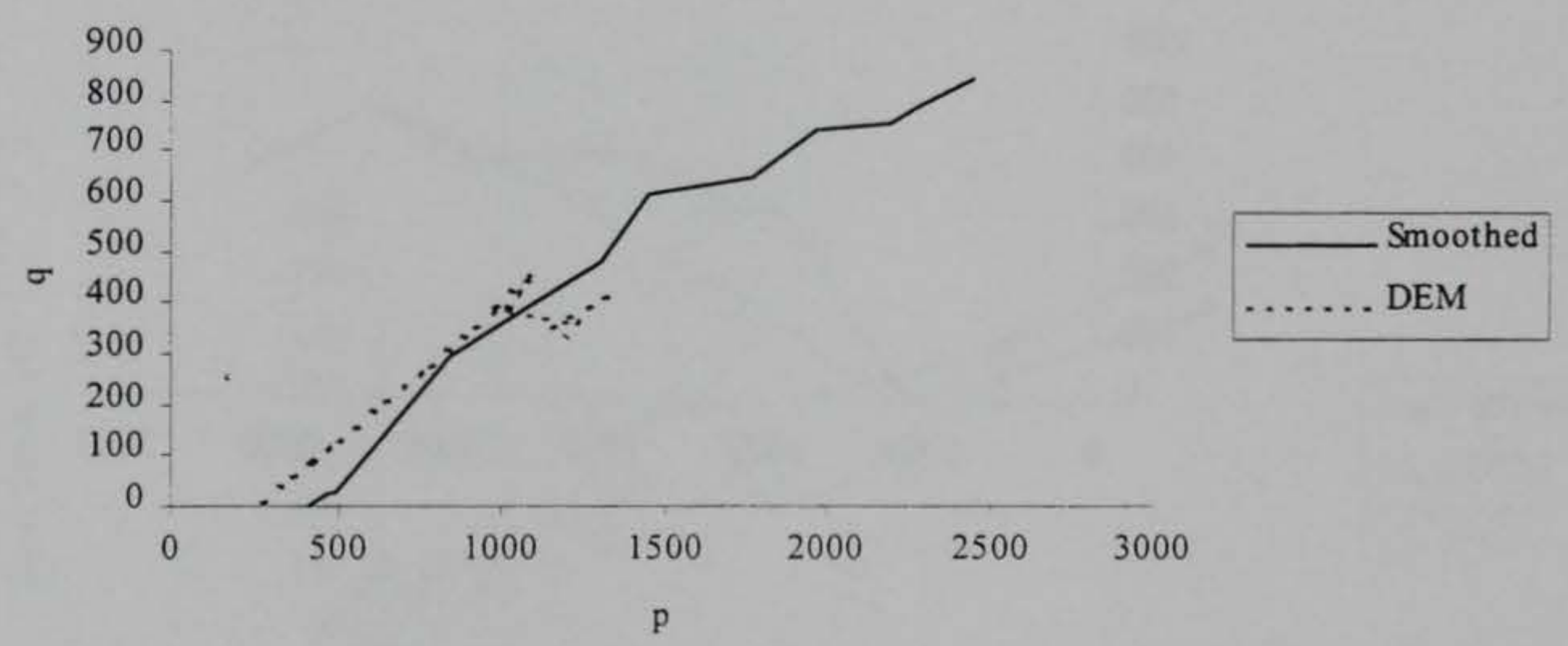

Figure 5.34: Comparison DEM and Smoothed System Stress Path for Point 9515 


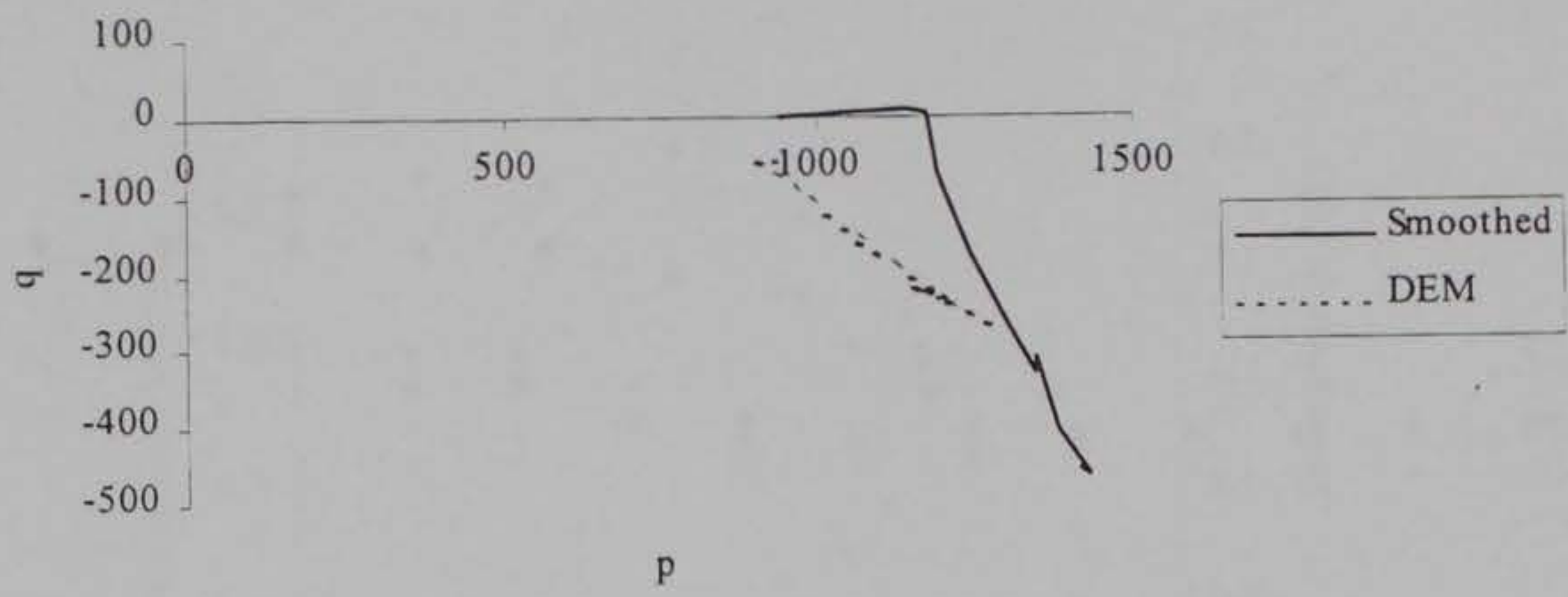

Figure 5.35: Comparison DEM and Smoothed System Stress Path for Point 9505

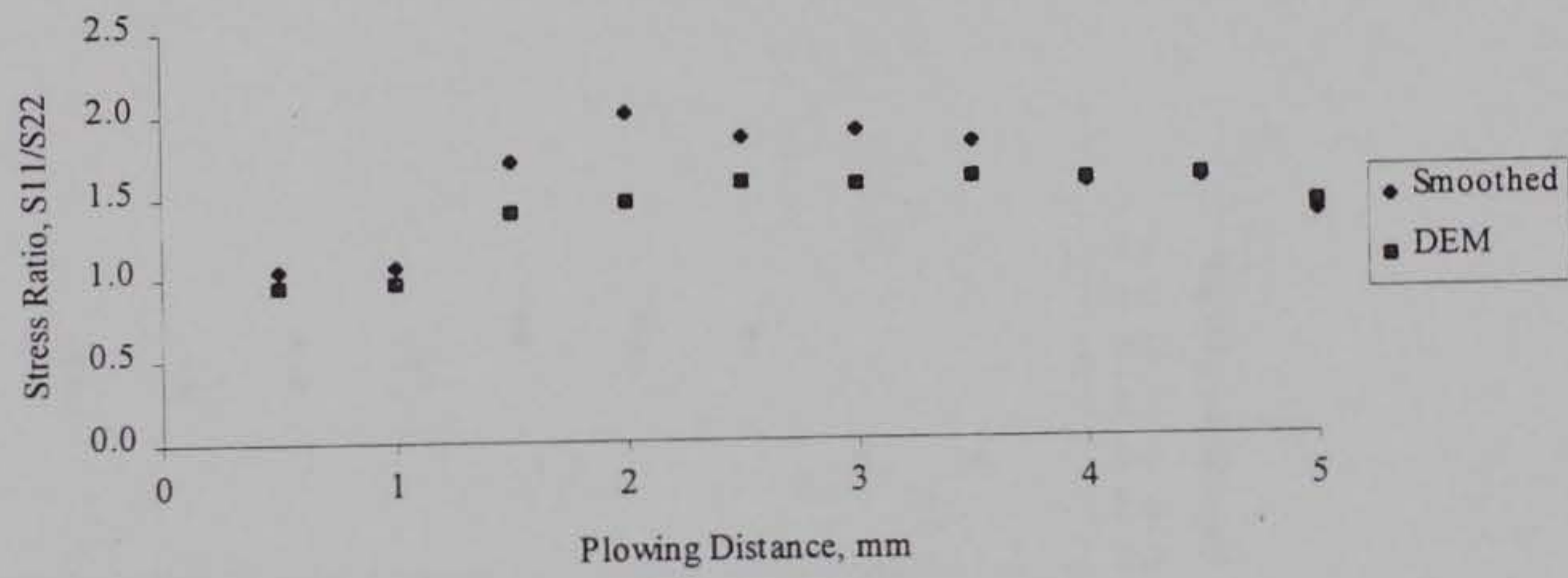

Figure 5.36: Comparison DEM and Smoothed System Stress Ratio for Point 9310 


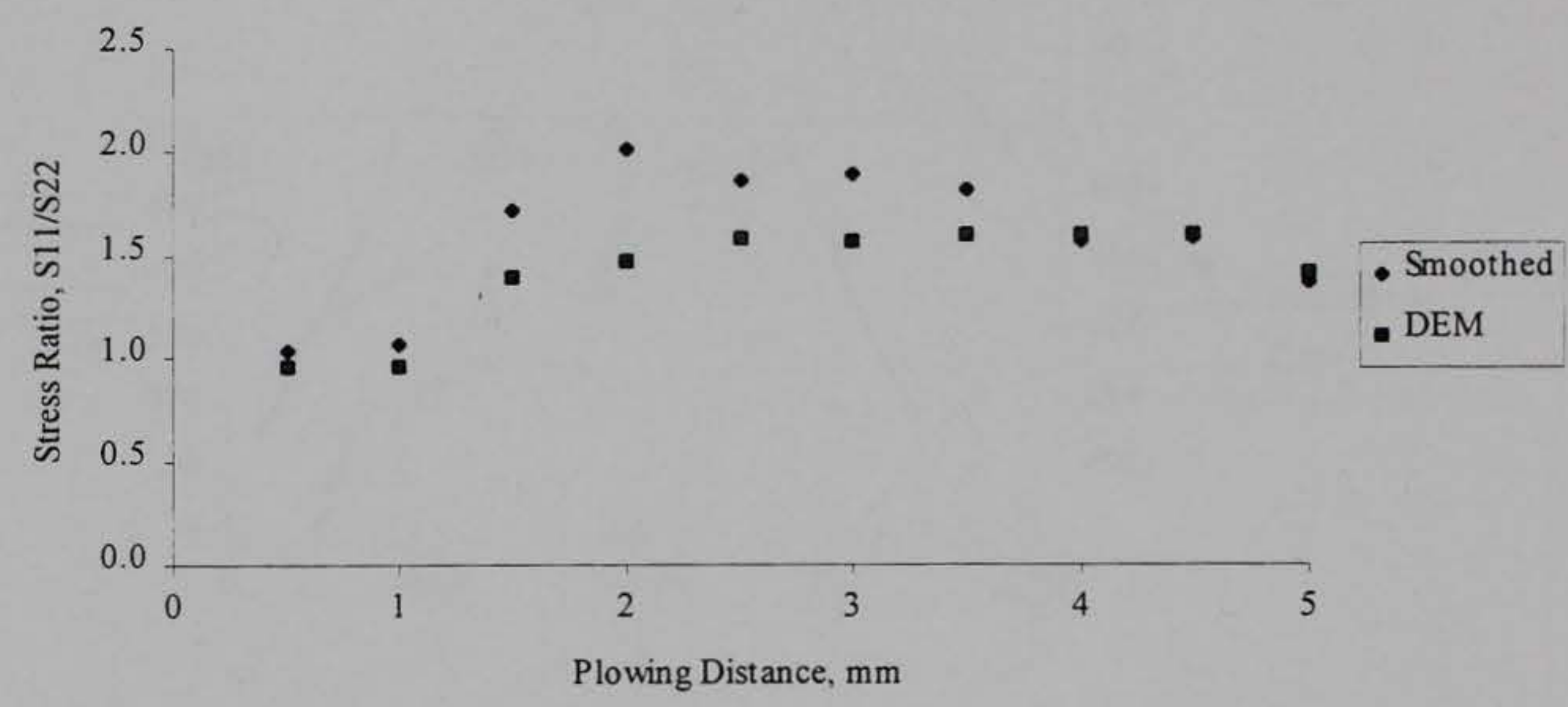

Figure 5.37: Comparison DEM and Smoothed System Stress Ratio for Point 9811

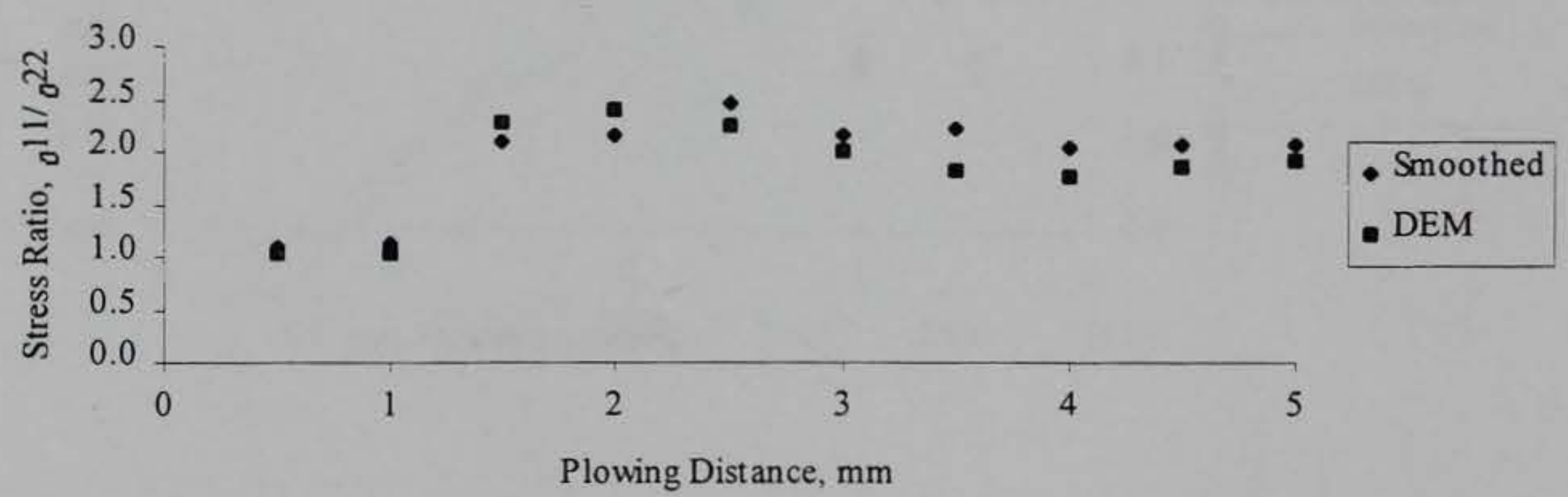

Figure 5.38: Comparison DEM and Smoothed System Stress Ratio for Point 9515 


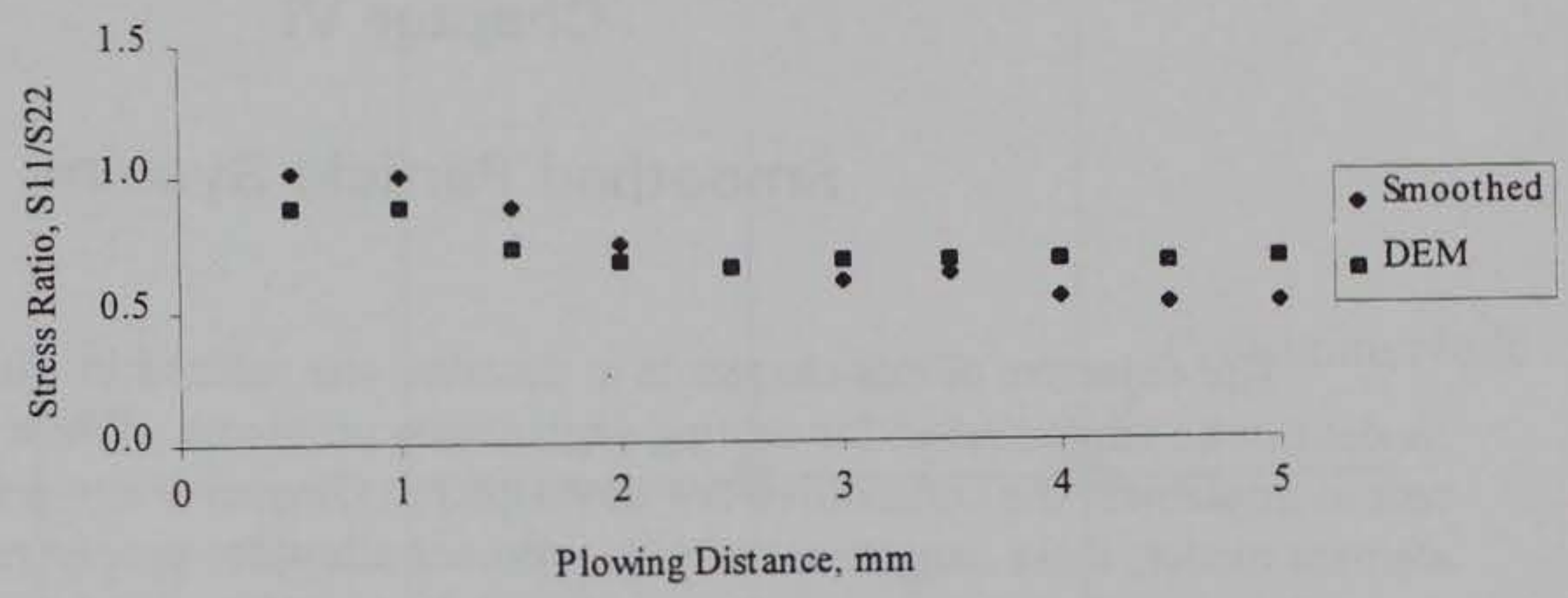

Figure 5.39: Comparison DEM and Smoothed System Stress Ratio for Point 9505 


\section{Chapter VI}

\section{Smoothed Particle System}

The objective of this chapter is to describe one method in which the smoothed particle model can be implemented for solving engineering problems. While it would be a fairly easy task to implement the constitutive law developed in Chapter 5 into a finite difference or finite element model, these numerical techniques do not allow for proper representation of the discontinuous soil deformations associated with large deformation problems. The new constitutive law was integrated into a numerical method similar to the particle-in-the-cell method used in smoothed particle hydrodynamic codes as described by Hockney and Eastwood [24]. The smoothed particle system consists of two parts, particles and a fixed grid, as shown in Figure 6.1. The particles are considered to be material points and store information about the current state of the system (i.e. density, stress). A fixed grid is placed over the problem space. The nodes of the grid are used as collection points where the particles are sampled to develop smoothed velocity and displacements of the system. These smoothed velocities and displacements are obtained by performing a spatial interpolation of the velocities and displacements of the surrounding particles as described in the descriptive averaging section of Chapter 3. The smoothed displacement field is applied back to the particles using a similar spatial interpolation to obtain the spatial velocity gradient tensor for the particle. The spatial velocity gradient tensor is used to update the stress acting at the material point.

Particles do not interact with the other particles directly but exchange state information with nodes of the fixed grid. The particles derive their motions from interpolation of the surrounding nodal velocities. The updated stress on the particle, which is used to update the forces acting on the nodes, is defined by a constitutive relationship that is derived from the contact information obtained from DEM simulations.

\subsection{Development of the Smoothed Particle System}

To illustrate the evolution of the smoothed particle system, a one-particle example is presented. In this example, only gravity is acting on the particle. For this example, the interpolation function will be defined as $\phi^{l}$, where a capital superscript indicates node number and a lower case superscript indicates particle number. A lower case subscript indicates direction. 


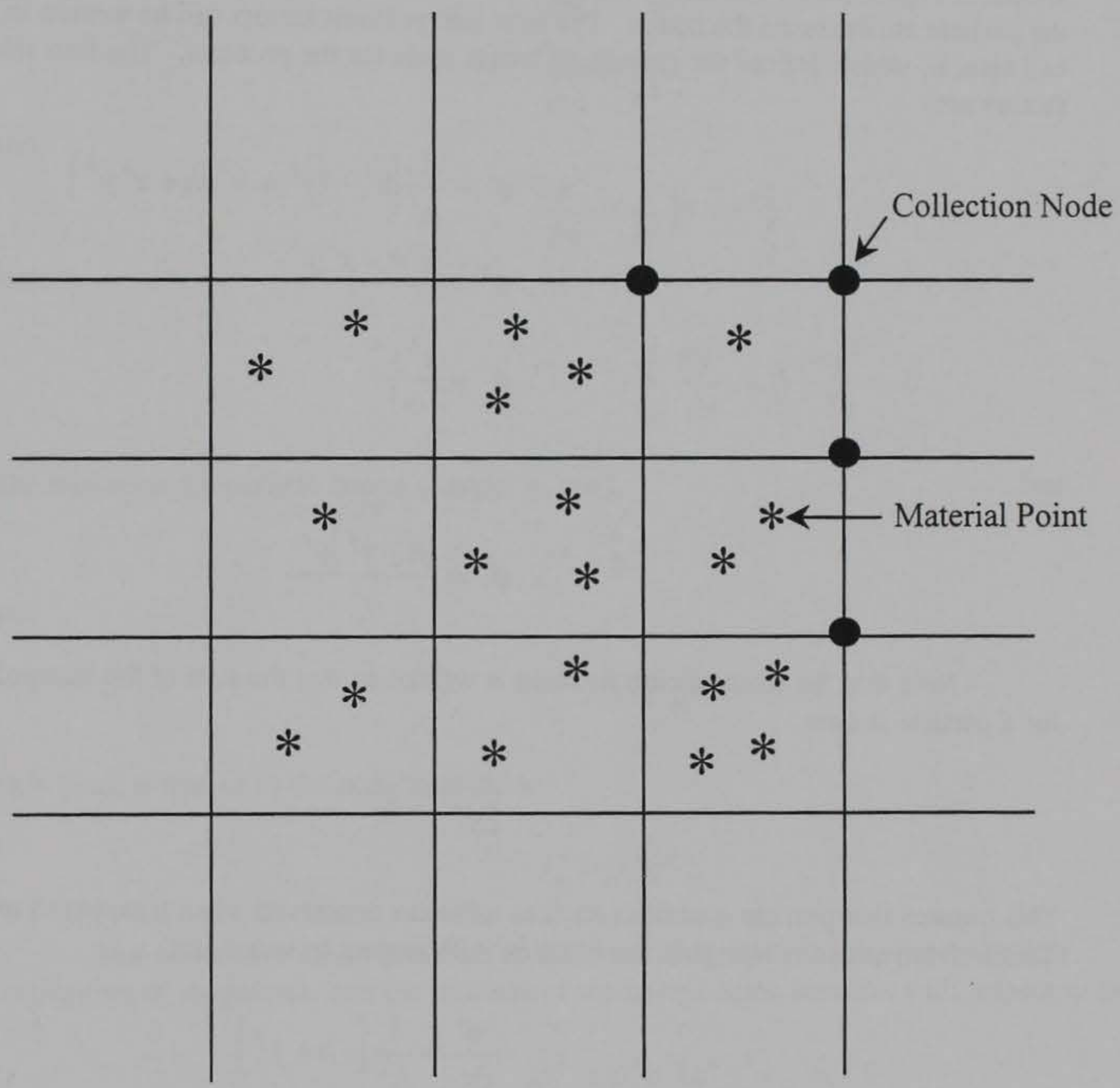

Figure 6.1: Smoothed Particle System 
In this example, a single particle is passing through a grid defined by four nodes as shown in Figure 6.2. A bi-linear interpolation function is used to determine the contribution of the particle attributes on the nodes. The four interpolation factors can be written in terms of the cell size, $\mathrm{h}$, which defines the smoothing length scale for the problem. The four interpolation factors are:

$$
\begin{aligned}
\phi^{1} & =\frac{1}{h^{2}}\left[h^{2}-\left(x^{k}+y^{k}\right) h+x^{k} y^{k}\right] \\
\phi^{2} & =\frac{x^{k} h-x^{k} y^{k}}{h^{2}} \\
\phi^{3} & =\frac{x^{k} y^{k}}{h^{2}}
\end{aligned}
$$

and

$$
\phi^{4}=\frac{\left(h-x^{k}\right) y^{k}}{h^{2}}
$$

Note that the interpolation function is written so that the sum of the interpolation factors for a particle is one:

$$
\sum_{I=1}^{4} \phi^{I}=1
$$

This ensures that particle quantities such as mass are conserved when transferred to the nodes. The derivatives of the interpolation function with respect to space are:

$$
\begin{aligned}
& \frac{\partial \phi^{1}}{\partial x}=\frac{1}{h^{2}}\left[-h+y^{k}\right] \\
& \frac{\partial \phi^{2}}{\partial x}=\frac{1}{h^{2}}\left[h-y^{k}\right] \\
& \frac{\partial \phi^{3}}{\partial x}=\frac{y^{k}}{h^{2}}, \\
& \frac{\partial \phi^{4}}{\partial x}=-\frac{y^{k}}{h^{2}},
\end{aligned}
$$




$$
\begin{aligned}
& \frac{\partial \phi^{1}}{\partial y}=\frac{1}{h^{2}}\left[-h+x^{k}\right] \\
& \frac{\partial \phi^{2}}{\partial y}=-\frac{x^{k}}{h^{2}} \\
& \frac{\partial \phi^{3}}{\partial y}=\frac{x^{k}}{h^{2}}
\end{aligned}
$$

and

$$
\frac{\partial \phi^{4}}{\partial y}=\frac{1}{h^{2}}\left[h-x^{k}\right]
$$

Note:

$$
\sum_{I-1}^{4} \frac{\partial \phi^{I}}{\partial x}=\sum_{I=1}^{4} \frac{\partial \phi^{I}}{\partial y}=0
$$

The forces on the particle due to gravity, $\mathrm{g}$, are

$$
f_{x}^{k}=0
$$

and

$$
f_{y}^{k}=-m^{k} g
$$

This force is moved to the node as follows:

$$
f_{y}^{I}=f_{y}^{k} \phi^{I}
$$

The convective momentum rate is allocated to the nodes as the product of the momentum of the particle and the gradient of the interpolation function with respect to time

$$
p_{i}^{I}=p_{i}^{k} \phi^{I}\left(x^{k} y^{k}\right)
$$

where

$$
\begin{aligned}
& p_{i}^{k}=m^{k} \sum_{I=1}^{4} \phi^{I}\left(x^{k} y^{y}\right) v_{i}^{I} \\
& \phi^{I}=\frac{\partial \phi^{I}}{\partial x} v_{x}^{k}+\frac{\partial \phi^{I}}{\partial y} v_{y}^{k} .
\end{aligned}
$$


The mass is lumped at the nodes:

$$
\begin{aligned}
& M^{I}=m^{k} \phi^{I} \\
& p_{i}^{n+\frac{1}{2}}=P_{i}^{n-\frac{1}{2}}+\frac{1}{M} P_{i} \Delta t
\end{aligned}
$$

The velocity is thus

$$
v_{i}^{n+\frac{1}{2}}=\frac{1}{M} P_{i}^{n+\frac{1}{2}}
$$

and the node displacement is

$$
d_{i}^{n}=v_{i}^{n+\frac{1}{2}} \Delta t
$$

The new location of the particles is interpolated from the nodal values of $d_{i}$

$$
d_{i}^{k}=\sum_{I=1}^{4} \phi^{I} d_{i}^{I}
$$

\subsection{Plow Simulation}

To demonstrate the application of the smoothed particle system, a plowing experiment similar to the one in Chapter 4 was performed. The material properties used in Chapter 5 to calibrate the smoothed particle model were used in this simulation. The simulation consisted of placing 2200 smoothed particles into a two-dimensional box with dimensions of $100 \mathrm{~mm}$ in the horizontal direction and $22 \mathrm{~mm}$ in the vertical direction. The plow moved at a velocity of 25 $\mathrm{mm}$ per second at a depth of $10 \mathrm{~mm}$. The grid had a node spacing of $5 \mathrm{~mm}$. Figure 6.3 shows the displacements of the smoothed particle at $5 \mathrm{~mm}, 10 \mathrm{~mm}$, and $20 \mathrm{~mm}$ of plowing. The colors in Figure 6.3 represent the horizontal particle velocities. The color map ranges from blue, which represents zero velocity to red, which represents a velocity of $25 \mathrm{~mm} / \mathrm{second}$. This figure can be compared to the results of the laboratory plowing experiment, Figures 4.7 and 4.10 in Chapter 4. From this figure, similarities can be seen between the smoothed particle system and the granular material. In general, the horizontal and vertical motion of the smoothed particles is similar to that of the real granular material; however, because of the coarseness of the computational grid, there tends to be far more smoothing of displacements in the smoothed particle system and the sharp shear band zone that formed in the soil experiments does not form in the smoothed particle system. The same experiment was performed using a node spacing of $2.5 \mathrm{~mm}$. Figure 6.4 shows the particle locations at 5 and $10 \mathrm{~mm}$ of plowing, when using the smaller grid. As expected, as the computational grid is refined, the smoothed particle system will behave more like the real granular material. Figure 6.5 shows an enlarged 
view of both the simulations. The vectors shown in this figure represent the amount of particle displacement relative to the particle's original position. By examining the region just below the plow, it can be clearly seen that there is more smearing of the displacements at the larger grid size. While the smaller grid size produces more realistic results, it should be pointed out that the critical time step for the system becomes smaller as the size of the grid is reduced.

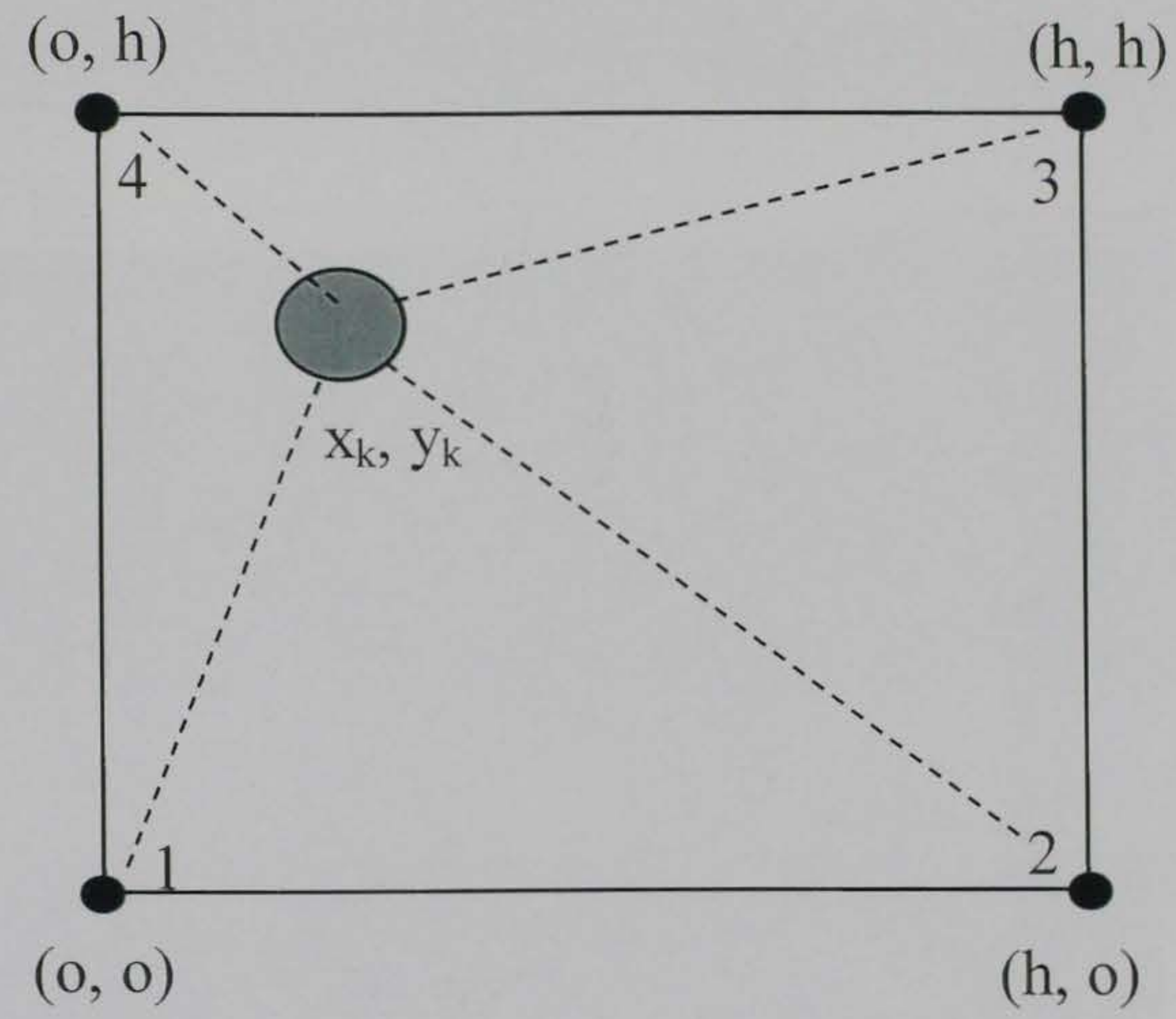

Figure 6.2: One Particle Example of a Smoothed Particle System 

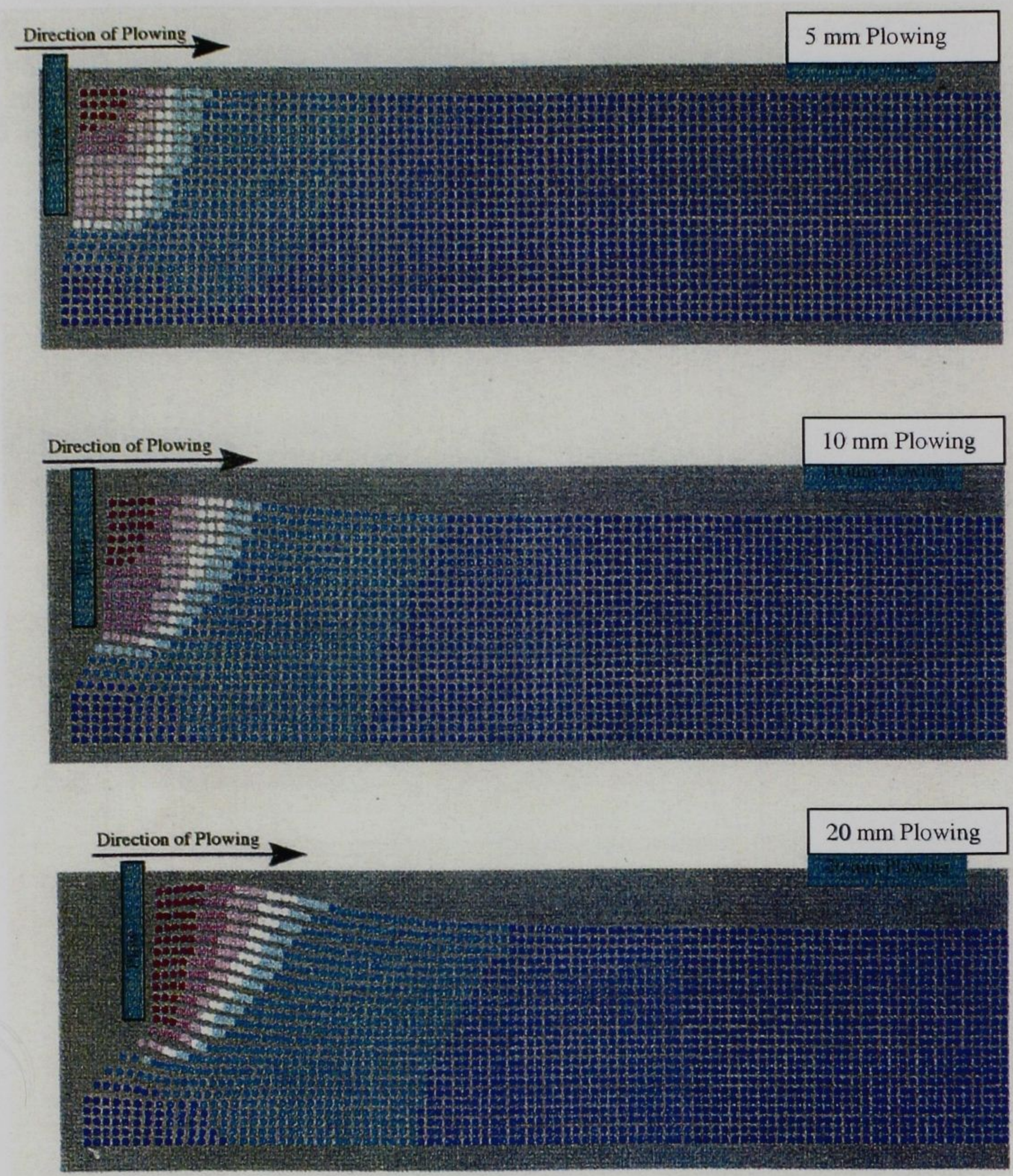

Figure 6.3: Smooth Particle System Plowing Simulation, Grid Size $=5 \mathrm{~mm}$ 

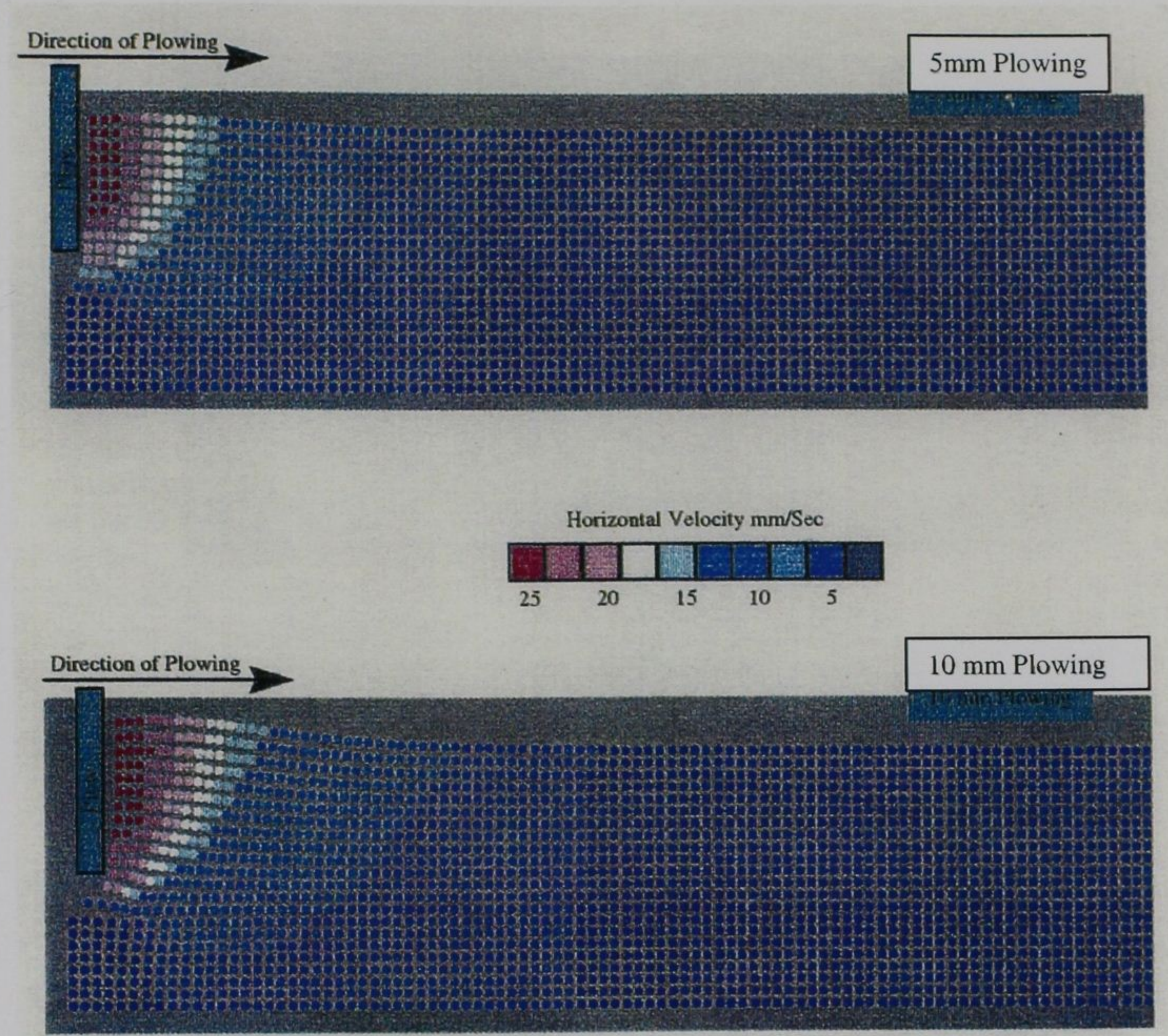

Figure 6.4: Smooth Particle System Plowing Simulation, Grid Size $=2.5 \mathrm{~mm}$ 


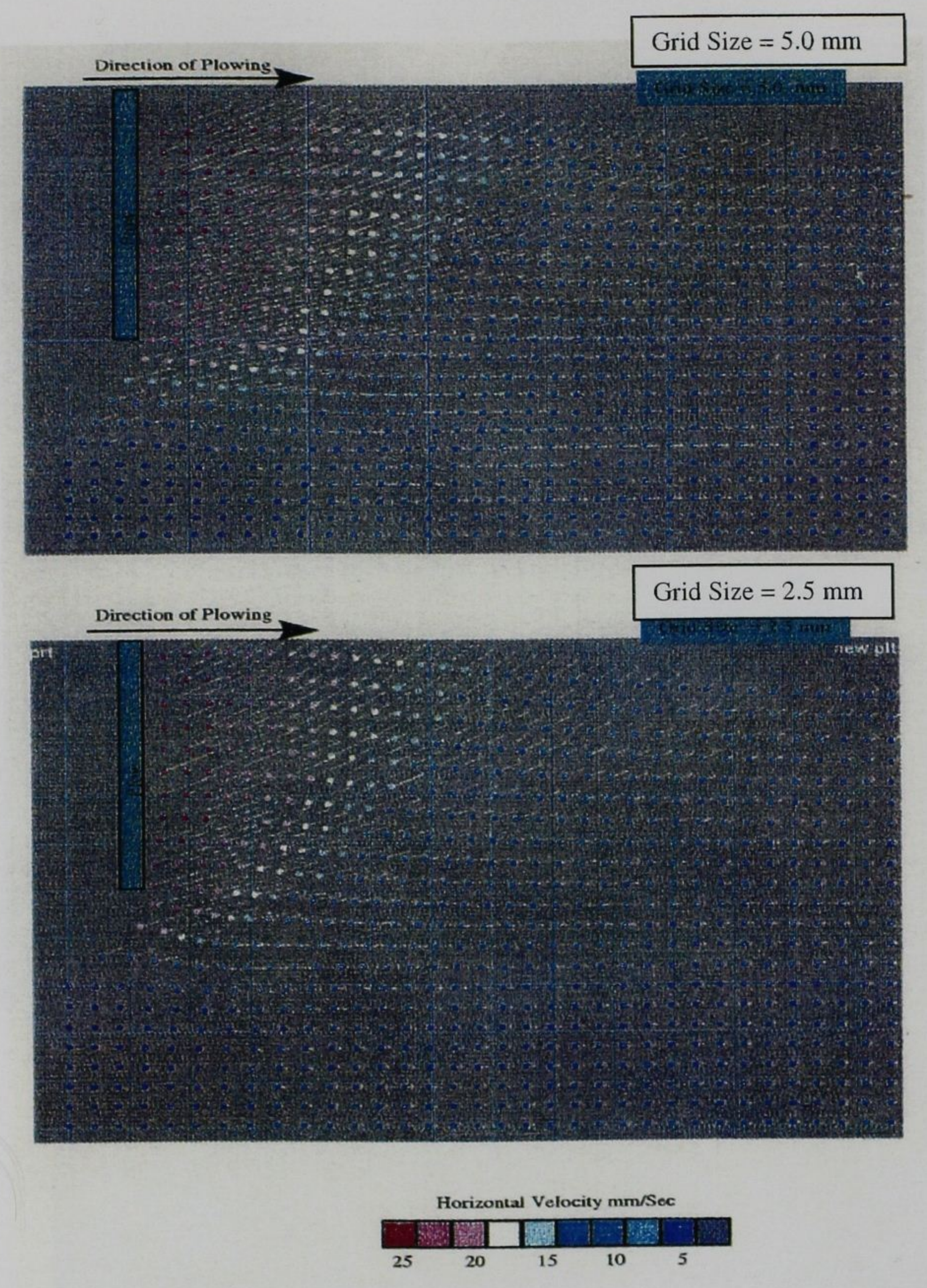

Figure 6.5: Effects of Grid Size on Particle Displacements 


\section{Chapter VII}

\section{Summary and Conclusions}

In Chapter 1, four research goals were established to develop a constitutive law for large deformation of granular material. The first goal was to develop a three dimensional discrete element model and improve computational efficiency of the discrete element model to allow for large simulations. The need for large particle simulations was in order to develop an ability to model laboratory experiments on a one-to-one basis so that the discrete element model could be evaluated against real soils. The second goal was to demonstrate that simulation of laboratory experiments by the discrete element model yields results comparable to real soils. Once established that the discrete element model provided a reasonable model of the real granular material, a third goal of establishing an averaging scheme to convert properties local to the particles (e.g. mass, momentum) into continuum attributes (e.g. density, velocity gradients) was met. From this averaging scheme a new constitutive law was developed to model large deformation of granular material. The fourth goal was to devise a computational procedure for modeling prototype-scale behavior using sparse particle systems for which computations of field scale problems can be performed on existing computer systems. The computational procedure used a particle scheme to ensure proper representation of the discontinuous soil deformations associated with large deformation problems.

\subsection{Significant Findings}

a) DEM comparison with Soil Experiments - A comparison was made between laboratory experiments involving very large discontinuous deformations in sand and numerical simulations, using a large-scale DEM computation. The magnitude of the simulation provides a unique opportunity to assess the validity of the DEM, based on experimental results. The simulation captures the behavior of a particulate "continuum" while the small-scale test permits a one-to-one correspondence between particle gradation in the simulation and the test. The agreement between the experimental and simulated particle motions in the plowing experiment indicates that many fine-grained details not captured by the simplistic particle interaction model may not be relevant in statistically large assemblies.

b) Continuum representation of a discrete system - An averaging process using the concept of an implied averaging volume was developed for obtaining continuum properties of a discrete system. Effects of sampling size were quantified, which lead to the conclusion that a significant sampling size (number of particles in the sample) is required to obtain meaningful macroscopic properties of the granular media.

c) Micro-Mechanical Modeling of Granular Media - The analysis began with consideration of a smoothing of the DEM quantities which amounts to application of a weighted residual approximation of the difference equations governing the DEM simulations. The smoothing process eliminates spatial detail and only the statistical descriptions of particle interactions are needed to evaluate the equations of motion for the smoothed system. The key 
to a continuum theory for granular media, therefore, is to relate the statistics of particle interactions to the kinematics of the smoothed system. This analysis implies that the macroscopic behavior of the discrete system could be approximated from a sampling of the contacts. This observation suggests that it could be possible to replace the highly detailed discrete particle system with a simpler model that represented an average particle. The average particle would maintain the same average properties of the total discrete system. It is concluded that without a micro-mechanical approach based on physical measurements, a satisfactory theory would be difficult to develop.

d) Non-Affine Motions - The evolution of contact properties is not readily determined from averaged particle movements because of non-affine components of particle interaction. A diffusion component was added to the smoothed model to account for the non-affine motions that are present in the granular media. The addition of the diffusion component made the statistics of the smoothed system behaves more like that of the DEM simulation.

\subsection{Need for Future Research}

The research presented in this dissertation was limited to dry, uniform, granular materials. Additional research should be conducted to quantify the effects that particle shape and grain size distribution has on the smoothed particle system. The smoothed particle system should be evaluated against geotechnical problems different from the plowing problem, to verify its application to more general problems. As computational capabilities improve, research should be conducted to model fine grained, clay particles.

Additionally, work should be conducted to include pore fluid into the system to allow for more realistic modeling of in-situ soil conditions. One of the predominant disadvantages with the traditional discrete element method is the inability to easily model a pore fluid. The inclusion of the pore fluid into the smoothed particle system should be comparable to a coupled deformation-fluid flow model derived from Biot's consolidation theory, because the smoothed particle system is defined as a continuum. 


\section{BIBLIOGRAPHY}

[1] Bardet, J.P. and Proubet, J. (1991) "A Numerical Investigation of the Structure of Persistent Shear Bands in Granular Media," Géotechnique, Vol. 41, No. 4, pp. 599-613.

[2] Barbosa, R.E. and Ghaboussi, J. (1990) "Discrete Finite Element Method for Multiple Deformable Bodies," Finite Elements in Analysis and Design, pp. 145-158.

[3] Bathurst, R.J. and Rothenburg, L. (1988) "Micromechanical Aspects of Isotropic Granular Assemblies With Linear Contact Interactions," Journal of Applied Mechanics, Vol. 15, March, pp. 17-23.

[4] Bažant, Z.P., Tabbara, M.R., Kazemi, M.T., and Pijaudier-Carbot, G. (1990) "Random Particle Model for Fracture of Aggregate or Fiber Composites," Journal of Engineering Mechanics, Vol. 116, No. 8, August, pp. 1687-1705.

[5] Bear, J. and Verruijt A. (1987) "Modeling Groundwater Flow and Pollution," D. Reidel Publishing Company, Dordrecht.

[6] Benz, W. (1988) "Applications of Smooth Particle Hydrodynamics (SPH) to Astrophysical Problems," Computer Physics Communications, Vol. 48, pp. 97-105.

[7] Benz, W. (1990) "Smooth Particle Hydrodynamics, a Review," The Numerical Modeling of Linear Stellar Pulsation's, Kluwer Academic Publishers, pp. 269-288.

[8] Carrillo, A.R., Horner, D.A., and Peters, J.F. (1996) "Design of a Large Scale Discrete Element Soil Model for High Performance Computing Systems," Proceedings of Supercomputing '96, November, 17-22, San Diego.

[9] Chapuis, R.P. (1976) "De la Structure Geometrique des Mileux Granulair en Relation avec leur Comportement Mecanique," Sc.D thesis, Department of Civil Engineering, Ecole Polytechnique of Montreal, Canada.

[10] Chang, C.S., Sundaram, S.S., and Misra, A. (1989) "Initial Moduli of Particulated Mass With Erictional Contacts," International Journal for Numerical and Analytical Methods in Geomechanics, Vol. 13, John Wiley and Sons, Ltd., pp. 629-644.

[11] Chang, C.S. (1992) "Micromechanics Modeling for Deformation and Eailure of Granular Material," Advances in Micromechanics of Granular Materials, Elsevier Science Publishers B.V., Amsterdam, pp. $251-260$. 
[12] Chistoffersen, J., Mehrabadl, M.M., and Nemat-Nasser, S. (1981) "A Micromechanical Description of Granular Material Behavior," Journal of Applied Mechanics, Vol. 48, pp. 339-344.

[13] Corkum, B.T. and Ting, J.T. (1986) The Discrete Element Method in Geotechnical Engineering, Publication 86-11, University of Toronto.

[14] Cundall, P.A. and Strack, O.D.L. (1979) "A Discrete Numerical Model for Granular Assemblies," Géotechnique, Vol. 29, No. 1, pp. 48-64.

[15] Cundal1, P.A. (1977) "The Incorporation of Simple Block Deformability into the Distinct Element Method," Dames and Moore Advanced Technology Group, London.

[16] Cundall, P.A., and Hart, R.D. (1985) "Development of Generalized 2$D$ and 3-D Distinct Element Programs for Modeling Jointed Rock," Miscellaneous Paper SL-85-1, U.S. Army Engineer Waterways Experiment Station.

[17] Cundall, P.A. (1988) "Computer Simulations of Dense Sphere Assemblies," Micromechanics of Granular Materials, Elsevier Science Publishers B.V. Amsterdam, pp. 113-160.

[18] Freitag, D.R. (1965) "A Dimensional Analysis of the Performance of Pneumatic Tires on Soft Soils," A dissertation submitted in partial fulfillment of the requirements for the degree of Doctor of Philosophy in Civil Engineering, Auburn University.

[19] Ghaboussi, J. and Barbosa, R. (1993) "Three-Dimensional Discrete Element Method for Granular Materials," International Journal for Numerical and Analytical Methods in Geomechanics, Vol. 14, John Wuler and Sons, Ltd., pp. 451-472.

[20] Gingold, R.A. and Monaghan, J.J. (1977) "Smoothed Particle Hydrodynamics: Theory and Application to Non-Spherical Stars," Monthly Notices of the Royal Astronomical Society, Vol. 181, pp. 375-389.

[21] Goodman, R.E., Taylor, R.L., and Brekke, T.L. (1968) "A Model for the Mechanics of Jointed Rocks," Journal of the Soil Mechanics and Foundation Divisions, American Society of Civil Engineers, 94(SM3), pp. 637-659.

[22] Grest, G.S., Dunweg, B., and Kremer, K. (1988) "Vectorized Link Cell Fortran Code for Molecular Dynamics Simulations for a Large Number of Particles," Comp. Phys. Comm., 55, pp. 269-285.

[23] Hockney, R.W., Goel, S.P., and Eastwood, J.W. (1974) "Quiet HighResolution Computer Models of a Plasma," J. Comp. Phys., 14, pp. 148-158.

[24] Hockney, R.W. and Eastwood, J.W. (1988) "Computer Simulation using Particles," Adam Hilger Publishing, Bristol. 
[25] Hopkins, M.A., Jenkins, J.T. and Louge, M.Y. (1992) "On the Structure of 3D Shear Flows," Advances in Micromechanics of Granular Materials, Elsevier Science Publishers, Amsterdam, pp. $271-279$.

[26] Horner, D.A., Peters, J., Howington, S., and Hryciw, R. (1994) "Effects of Grain Size Distribution on the Physics of Particle Materials," Report No 94-9, Mechanics and Statistical Physics of Particulate Material Workshop, Institute for Mechanics and Materials, University of California, San Diego, pp. 86-89.

[27] Huang, A.B., Ma, M.Y., and Lee, J.S. (1992) "A Micromechanical Study of Penetration Tests in Granular Material," Advances in Micromechanics of Granular Materials, Elsevier Science Publishers, Amsterdam, pp. 173-181.

[28] Kruyt, N.P. (1993) "Towards Micro-Mechanical Constitutive Relations for Granular Materials," Micromechanics of Granular Materials, Elsevier Science Publishers, Amsterdam, pp. 147-178.

[29] Libersky, L.D., Petschek, A.G., Carney, T.C., Hipp, J.R., and Allahdadi, F.A. (1993) "High Strain Lagrangian Hydrodynamics," Journal of Computational Physics, Vol. 108.

[30] Marsal, R.J. (1973) "Mechanical Properties of Rockfill," Embankment Dam Engineering, pp.109-145.

[31] Miller, R.H. (1992) "Experimenting with Galaxies," American Scientist, April, pp. 152-163.

[32] Monagahan, J.J. (1982) "Why Particle Methods Work," Siam Journal on Scientific and Statistical Computing, Vol. 3, No. 4, pp. 422-433.

[33] Monagahan, J.J. (1985) "Particle Methods for Hydrodynamics," Computer Physics Reports 3, North-Holland Physics Publishing, pp. $71-124$.

[34] Monagahan, J.J. (1988) "An Introduction to SPH" Computer Physics Communications, Vol. 48, pp. 89-96.

[35] Monagahan, J.J. (1992) "Smoothed Particle Hydrodynamics," Annual Review of Astronomy Astrophysics, Vol. 30, pp. 543-574.

[36] Ng, T. P. (1989) "Numerical Simulation of Granular Soil Under Monotonic and Cyclic Loading: A Particulate Mechanics Approach," A dissertation submitted in partial fulfillment of the requirements for the degree of Doctor of Philosophy in Civil Engineering, Rensselaer Polytechnic Institute.

[37] Ng, T. P. and Dobry, R. (1992) "A Non-Linear Numerical Model for Soil Mechanics," International Journal for Numerical and Analytical Methods in Geomechanics, Vol.16, pp. 247-263. 
[38] Oda, M. (1977) "Co-ordination Number and its Relationship to Shear Strength of Granular Material," Soils and Foundations, Vol.17, pp. 29-42.

[39] Pan, X.D., and Reed, M.B. (1991) "A Coupled Distinct Element Finite Element Method for Large Deformation Analysis of Rock Masses," International Journal of Rock Mechanics Mining Sciences and Geomechanical Abstracts, Vol. 28, No. 1, pp. 93-99.

[40] Plimpton, S. (1993) "Fast Parallel Algorithms for Short-Range Molecular Dynamics," SAND91-1144, Sandia National Laboratories, May.

[41] Raschke, S. A., Hryciw, R.D., and Donohoe, G.D. (1996) "MicroDeformations in Sands by Digital Image Processing and Analysis." Transportation Research Record No. 1548, pp. 31-37.

[42] Rong, G.H., Negi, S.C., and Jofriet, J.C. (1995) "Simulation of Flow Behavior of Bulk Solids in Bins. Part 1: Model Development and Validation," Journal of Agricultural Engineering Research, Vol. 62, pp. $247-256$.

[43] Rowell, J.D. (1994) "Validation of Ellipse-Based Discrete Element Modeling in Soil Mechanics," A dissertation submitted in partial fulfillment of the requirements for the degree of Master of Science in Civil Engineering, University of Massachusetts, Lowell.

[44] Sakaguchi, H. and Ozaki, E. (1993) "Analysis of the Formation of Arches Plugging the Flow of Granular Materials," Powders and Grains 93, Balkema, Rotterdam, pp. 351-356.

[45] Shukla, A. and Sadd, M.H. (1990) "Wave Propagation and Dynamic Load Transfer Due to Explosive Loading in Heterogeneous Granular Media with Microstructure," Prepared for U.S. Air Force Office of Scientific Research, Contract No. F49620-89-C-0091, Bolling Air Force Base, Bolling, DC.

[46] Ting, J.M., Corkum, B.T, Kauffman, K.R., and Greco, C. (1987) "Discrete Numerical Modeling of Soil: Validation and Application," Publication No. 87-03, University of Toronto, June.

[47] Ting, J.M. and Corkum, B.T. (1988) "Strength Behavior of Granular Materials Using Discrete Numerical Modeling," Numerical Methods in Geomechanics, Balkema, Rotterdam, pp. 305-310.

[48] Ting, J.M., Corkum, B.T., Kauffman, C.R., and Greco, C. (1989) "Discrete Numerical Model for Soil Mechanics," Journal of Geotechnical Engineering, Vol. 115, No. 3, pp. 379-398.

[49] Ting, J.M., Khwaja, M., Meachum, L., and Rowell, J. (1993) "The Ellipse-Based Discrete Element Model for Granular Materials," International Journal for Numerical and Analytical Methods in Geomechanics, Vol. 17, Wiley Publishers, Chichester, pp. 603-623. 
[50] Ting, J.M., Meachum, L., and Rowell, J.D. (1994) "Effect of Particle Shape on the Strength and Deformation Mechanisms of Ellipse-Shaped Granular Assemblages," Engineering Computations, Vol. 12, Pineridge Press Limited, pp. 19-108.

[51] Valanis, K. C. (1971) "A Theory of Viscoplasticity Without a Yield Surface, Part I, General Theory," Archives of Mechanics, Vol. 23, pp. 517-533.

[52] Valanis, K. C. and Peters, J. F. (1993) "Ill-Posedness of the Initial and Boundary Value Problems in Non-Associative Plasticity," Acta Mechanica, Vol. 114, pp. 1-25.

[53] Verlet, L. (1967) "Computer Experiments on Classical Fluids: I. Thermodynamical Properties of Lennard-Jones Molecules," Phys. Rev, 159, pp. 98-103.

[54] Yanagisawa, E. (1983) "Influence of Void Ratio and Stress Condition on the Dynamic Shear Modules of Granular Media," Advances in the Mechanics and the Flow of Granular Material, Vol. 2, pp. 947-960.

[55] Zienkiewicz, O. C. (1977) "The Finite Element Method," McGraw-Hill Book Company, London, page 765 . 


\section{APPENDIX A}

\section{Dimensional Analysis of Particle System}

\section{A.1 Development of $\pi$-Numbers}

A $\pi$-number is a dimensionless number that is developed from a collection of key parameters that describe a physical process of interest. The $\pi$-number is used to identify proper scaling relationships for maintaining similitude between a prototype model and the fullscale system. This section describes a method for determining $\pi$-numbers for a system and has been adapted from the presentation of Freitag [18].

The system being evaluated can be described by a set of physical parameters, $P_{1}, P_{2}, \cdots P_{n}$, the fundamental dimensions are $d_{1,}, d_{2}, \cdots d_{r}$ so that, dimensionally:

$$
p_{s} \underline{\underline{D}} d_{1}^{e_{1 s}} d_{2}^{e_{2 s}} \cdots d_{r}^{e_{r s}}
$$

where the symbol $\underline{\underline{D}}$ means "dimensional equal to" and the $e_{r s}$ are the components indicating the multiplicity of each dimension in the parameter.

For example, the parameter mass, $m$, is dimensionally equal to

$$
m \stackrel{D}{=} F L^{-1} T^{2}
$$

where $\mathrm{F}, \mathrm{L}$, and $\mathrm{T}$ represent the dimensions force, length, and time.

The $\pi$-number is developed by combining the parameters in such a way as to yield a dimensionless number. 


$$
\pi=P_{1}^{x_{1}} P_{2}^{x_{2}} P_{3}^{x_{3}}
$$

To obtain a dimensional relationship, the sum of the exponents of each dimension must be zero. Thus,

$$
\begin{aligned}
& e_{11} x_{1}+e_{12} x_{2}+e_{13} x_{3} \cdots e_{1 n} x_{n}=0 \\
& e_{21} x_{1}+e_{22} x_{2}+e_{23} x_{3} \cdots e_{2 n} x_{n}=0 \\
& e_{r 1} x_{1}+e_{r 2} x_{2}+e_{r 3} x_{3} \ldots e_{m} x_{n}=0
\end{aligned}
$$

where the number of dimensions, $\mathrm{r}$, is less than the number of parameters, $\mathrm{n}$.

The values of the $e_{i j}$ are known from the nature of the parameters, but the $x_{i}$ must be found. The set of equations representing the relations among the unknowns $x_{i}$ is governed by rules of matrix algebra. The coefficients of the $x_{i}$ from a matrix of particular rank, with the columns representing the constitution of each parameter and the rows, the frequency of each dimension as indicated.

$$
\begin{array}{c|cccc|} 
& p_{1} & p_{2} & \cdots & p_{n} \\
\hline d_{1} & e_{11} & e_{12} & \cdots & e_{1 n} \\
d_{2} & e_{21} & e_{22} & \cdots & e_{2 n} \\
\vdots & \vdots & \vdots & \vdots & \vdots \\
d_{r} & e_{r 1} & e_{r 2} & \cdots & e_{m}
\end{array}
$$

There are n-r more unknowns than equations, so the solution consists of expressions for $\mathrm{r}$ of the unknowns, $x_{i}$, in terms of the other n-r unknowns. To ensure that the matrix solution used here will provide the maximum number of linearly independent solutions, the equations and the unknowns must be arranged to obtain a non-zero determinant in the upper left corner of the matrix. Standard matrix manipulation is used to obtain an $\mathrm{r} \times \mathrm{r}$ identity submatrix, as shown below:

\begin{tabular}{r|cccccccc} 
& $p_{1}$ & $p_{2}$ & $p_{3}$ & $\cdots$ & $p_{r+1}$ & $p_{r+2}$ & $\cdots$ & $p_{n}$ \\
\hline$d_{1}$ & 1 & 0 & 0 & $\cdots$ & $C_{1, r+1}$ & $C_{1, r+2}$ & $\cdots$ & $C_{1 n}$ \\
$d_{2}$ & 0 & 1 & 0 & $\cdots$ & $C_{2, r+1}$ & $C_{2, r+2}$ & $\cdots$ & $C_{2 n}$ \\
$d_{3}$ & 0 & 0 & 1 & $\cdots$ & $C_{3, r+1}$ & $C_{3, r+2}$ & $\cdots$ & $C_{3 n}$ \\
$\vdots$ & $\vdots$ & $\vdots$ & $\vdots$ & $\ddots$ & $\vdots$ & $\vdots$ & $\ddots$ & $\vdots$ \\
$d_{r}$ & 0 & 0 & 0 & $\cdots$ & $C_{r, r+1}$ & $C_{1, r+2}$ & $\cdots$ & $C_{m}$
\end{tabular}


Each row of the solution matrix yields an expression of one of the $\mathrm{r}$ parameters in terms of the remaining ( $n-r)$ parameters. The solution can been obtained by means of elementary simultaneous equations to yield the following expressions.

$$
\begin{aligned}
& x_{1}=C_{1, r+1} x_{r+1}+C_{1, r+2} x_{r+2}+\ldots C_{1 n} x_{n} \\
& x_{2}=C_{2, r+1} x_{r+1}+C_{2, r+2} x_{r+2}+\ldots \quad C_{2 n} x_{n} \\
& \begin{array}{ccc}
. & & \vdots \\
. & . & \vdots \\
. & . & \vdots
\end{array} \\
& x_{r}=C_{r, r+1} x_{r+1} \quad C_{r, r+2} x_{r+2}+\ldots \quad C_{2 n} x_{n}
\end{aligned}
$$

Assigning the values $x_{r+1}=1$ and all others, $x_{r+2}, \ldots x_{n}=0$ for the first solution, it is found from Equation A.7 that $x_{1}=\bullet C_{1, r+1}, x_{2}=C_{2, r+2}, \ldots x_{r}=C_{r, r+1}$. Similarly, if $x_{r+1}=0$ and $x_{r+2}=1$, all $x_{r+2}$ to $x_{n}=0$, it is found that $x_{1}=C_{1, r+2} x_{2}=C_{2, r+2, \ldots x_{r}}=C_{r, r+2}$. This process can be continued until all (n-r)x terms from $x_{r}$ to $x_{n}$ have successively been assigned the value of 1 . These results can be arranged in matrix form.

\begin{tabular}{c|cccccccc} 
& $x_{1}$ & $x_{2}$ & $x_{3}$ & $\cdots$ & $x_{r+1}$ & $x_{r+2}$ & $\cdots$ & $x_{n}$ \\
& $p_{1}$ & $p_{2}$ & $p_{3}$ & $\cdots$ & $p_{r+1}$ & $p_{r+2}$ & $\cdots$ & $p_{n}$ \\
\hline$\pi_{1}$ & $C_{1, r+1}$ & $C_{2, r+1}$ & $C_{3, r+1}$ & $\cdots$ & 1 & 0 & $\cdots$ & 0 \\
$\pi_{2}$ & $C_{1, r+2}$ & $C_{2, r+2}$ & $\vdots$ & $\cdots$ & 0 & 1 & $\cdots$ & 0 \\
$\pi_{1}$ & $C_{1, r+3}$ & $C_{2, r+3}$ & $\vdots$ & $\cdots$ & 0 & 0 & $\cdots$ & 0 \\
$\vdots$ & $\vdots$ & $\vdots$ & $\vdots$ & $\vdots$ & $\vdots$ & $\vdots$ & $\vdots$ & $\vdots$ \\
$\pi_{(n-r)}$ & $C_{1, n}$ & $C_{2, n}$ & $\vdots$ & $\cdots$ & 0 & 0 & $\cdots$ & 1
\end{tabular}

The submatrix on the right is an (n-r) identity matrix. This submatrix has a nonzero determinant, so the rank of the matrix is n-r, which is equal to the number of rows.

Consequently, the rows in the matrix are linearly independent, and the rows constitute a fundamental system of solutions. The numerical values in each row are the set of exponents of the corresponding parameters that make up the $\pi$ term, thus

$$
\pi_{m}=p_{1}^{C_{1, r+m}} p_{2}^{C_{2, r+m}} p_{3}^{C_{3, r+m}} \cdots p_{r+m}
$$

where $1 \leq m \leq n-r$.

By inspection, the equation of $\pi$ terms (Equation A.9) can be written from the matrix of solutions (Equation A.7). The matrix of the coefficients in their first $r$ columns of Equation A.9 is simply the transpose of the $\mathrm{r} x(\mathrm{n}-\mathrm{r})$ submatrix on the right of Equation A.7. Note, however, that the signs of all coefficients must change at the same time to be equivalent to the 
operation represented by Equation A.8. This matrix is then simply augmented by the (n-r) $\mathrm{x}$ (n-r) identity to yield the form shown as Equation A.9.

\section{Application of $\pi$-Theorem to the Soil Particle System}

Eight parameters were chosen to describe the soil particle system. These parameters included stress, gravitational constant, time, density, mass, particle stiffness, particle viscous damping coefficient, and particle diameter.

These 8 parameters can be recorded with their dimensions in matrix form as follows:

\begin{tabular}{l|cccccccc} 
& $\sigma$ & $g$ & $T$ & $\gamma$ & $m$ & $k$ & $c$ & $d$ \\
\hline$F$ & 1 & 0 & 0 & 1 & 1 & 1 & 1 & 0 \\
$L$ & -2 & 1 & 0 & -3 & -1 & -1 & -1 & 1 \\
$T$ & 0 & -2 & 1 & 0 & 2 & 0 & 1 & 0
\end{tabular}

The determinant of the matrix is non-zero, so the rank of the matrix is three. Therefore, eight parameters minus the rank of three yield five $\pi$ terms that will describe the system. By using the method described in the previous section, the five $\pi$ terms can be derived as:

\begin{tabular}{l|rrrrrrrr} 
& $\sigma$ & $g$ & $T$ & $\gamma$ & $m$ & $k$ & $c$ & $d$ \\
\hline$\pi_{1}$ & -1 & 1 & 2 & 1 & 0 & 0 & 0 & 0 \\
$\pi_{2}$ & -1 & -1 & -4 & 0 & 1 & 0 & 0 & 0 \\
$\pi_{3}$ & -1 & -1 & -2 & 0 & 0 & 1 & 0 & 0 \\
$\pi_{4}$ & -1 & -1 & -3 & 0 & 0 & 0 & 1 & 0 \\
$\pi_{5}$ & 0 & -1 & -2 & 0 & 0 & 0 & 0 & 1
\end{tabular}

Thus,

$$
\pi_{1}=\frac{g T^{2} \gamma}{\sigma}, \pi_{2}=\frac{m}{\sigma g T^{4}}, \pi_{3}=\frac{k}{\sigma g T^{2}}, \pi_{4}=\frac{C}{\sigma g T^{3}}, \pi_{5}=\frac{d}{g T^{2}}
$$

\section{Use of $\pi$-numbers for Scaling Relationships}

Because a $\pi$ number is dimensionless, it can be combined with other $\pi$ numbers to produce other dimensionless relationships among the parameters. For example, dividing $\pi_{3}$ by $\pi_{2}$ yields: 


$$
\pi=\frac{m}{T^{2} k}
$$

which by rearranging the terms will produce the form of the equation for the critical time step of the system.

$$
T=\sqrt{\frac{m}{\pi k}}
$$

Another interesting relationship occurs by dividing $\pi_{5}$ by $\pi_{3}$ to get

$$
\pi=\frac{k}{d \sigma}
$$

This equation can be combined with the parameter strain, $\varepsilon$, which is a dimensionless number, to develop a stress-strain dimensional relationship.

$$
\sigma=\frac{\pi k}{d} \varepsilon
$$

where $\frac{\pi k}{d}$ could be defined as a Young's modulus for the media. This indicates that for a given material, i.e. quartz, the contact spring constant must vary with the radius of the particle. Additionally, because the mass of a particle is proportional to the cube of the particle radius, equation A.14 implies that the critical time step will become smaller as smaller particles are used.

A typical use of $\pi$-numbers in geotechnical engineering is in the development of centrifuge scaling laws. In a centrifuge test, the prototype model is subject to a large gravitational force. This change, $\alpha$, is represented by

$$
\alpha=\frac{g_{\text {protype }}}{g_{\text {fullscale }}}
$$


Table A.1: Scaling Relationships for Centrifuge Test

Quantity

Length, $d$

Contact stiffness, $\mathrm{k}$

Viscous damping, $\mathrm{c}$

Mass of particle

Unit weight

Mass density

Time

Stress

Strain
Full Scale

1

1

1

1

1

1

1

1

1
Centrifugal Model a $\alpha$ g's

$1 / \alpha$

$1 / \alpha$

$1 / \alpha^{2}$

$1 / \alpha^{3}$

$\alpha$

1

$1 / \alpha$

1

1

The objective of the centrifuge scaling laws is to maintain stress similitude between the prototype and the full-scale system (i.e., $\sigma_{p}=\sigma_{f s}$ ). The Table A.1 summarizes the scaling relationship for the particle system to a centrifugal test.

The dimensional analysis was originally performed to determine if scaling laws could be used to increase the critical time step while maintaining stress similitude. However, the results of the dimensional analysis indicate that scaling laws do not provide any advantage to modeling the system. 


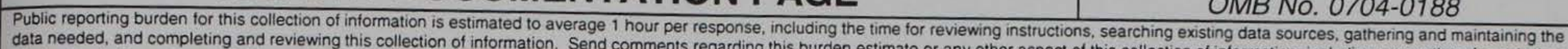

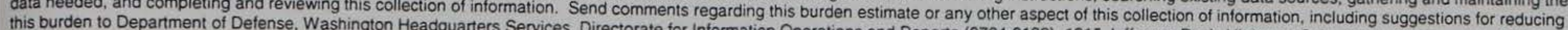

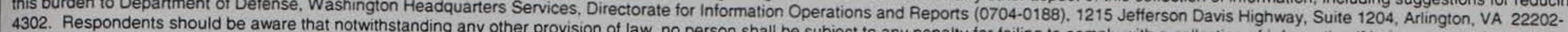

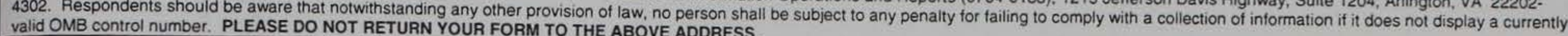
1. REPORT DATE (DD MM-YYYY)
August 2000

\section{REPORT TYPE}
Final report
3. DATES COVERED (From - To)

4. TITLE AND SUBTITLE

Application of DEM to Micro-Mechanical Theory for Large Deformations of Granular Media

\section{AUTHOR(S)}

David A. Horner and John F. Peters

5a. CONTRACT NUMBER

5b. GRANT NUMBER

5c. PROGRAM ELEMENT NUMBER

5d. PROJECT NUMBER

5e. TASK NUMBER

5f. WORK UNIT NUMBER

8. PERFORMING ORGANIZATION REPORT NUMBER

ERDC/GL TR-00-7

Geotechnical Laboratory

3909 Halls Ferry Road

Vicksburg, MS 39180-6199

9. SPONSORING / MONITORING AGENCY NAME(S) AND ADDRESS(ES)

U.S. Army Engineer Research and Development Center

3909 Halls Ferry Road

Vicksburg, MS 39180-6199

11. SPONSOR/MONITOR'S REPORT NUMBER(S)

\section{DISTRIBUTION / AVAILABILITY STATEMENT}

Approved for public release; distribution is unlimited.

\section{SUPPLEMENTARY NOTES}

\section{ABSTRACT}

A constitutive theory is developed for granular material undergoing arbitrarily large deformations. A three-dimensional discrete element model (DEM) was developed to simulate granular material. The computational efficiency of the DEM was improved to allow for modeling of large particle systems. The need for large particle simulations was to develop on ability to model laboratory experiments on a one-to-one basis so that the discrete elements model could be evaluated against real soils. A comparison was made between laboratory experiments involving very large discontinuous deformations in sand and numerical simulations using large-scale DEM computation. The magnitude of the simulation provided a unique opportunity to assess the validity of the DEM, based on experimental results. The agreement between the experimental and simulated particle motions in the plowing experiment indicates that details not captured by the simplistic particle interaction model may not be relevant in statistically large assemblies. Once it was established that the discrete element method provided a reasonable model for real granular material, an averaging scheme was developed to convert properties local to the particles (e.g., mass, momentum) into continuum attributes (e.g., density, velocity gradients). From this averaging scheme, a new constitutive law was developed to model large deformation of granular material.

\section{SUBJECT TERMS}

Discrete element modeling, Large deformation, Numerical modeling

\section{SECURITY CLASSIFICATION OF:}

\begin{tabular}{|l|l|l|}
\hline a. REPORT & b. ABSTRACT & c. THIS PAGE \\
UNCLASSIFIED & UNCLASSIFIED & UNCLASSIFIED \\
\hline
\end{tabular}

17. LIMITATION OF ABSTRACT

18. NUMBER
OF PAGES
133

19a. NAME OF RESPONSIBLE PERSON 19b. TELEPHONE NUMBER (include area code) 